\title{
Singular Integrals Associated with Zygmund Dilations
}

\author{
Yongsheng $\mathrm{Han}^{1} \cdot \mathrm{Ji} \mathrm{Li}^{2}\left(\mathbb{D} \cdot\right.$ Chin-Cheng $\mathrm{Lin}^{3}$. Chaoqiang $\operatorname{Tan}^{4}$
}

Received: 20 May 2018 / Published online: 23 August 2018

(c) The Author(s) 2018

\begin{abstract}
The main purpose of this paper is to study multi-parameter singular integral operators which commute with Zygmund dilations. Motivated by some explicit examples of singular integral operators studied in Ricci and Stein (Ann Inst Fourier (Grenoble) 42:637-670, 1992), Fefferman and Pipher (Am J Math 11:337-369, 1997), and Nagel and Wainger (Am J Math 99:761-785, 1977), we introduce a class of singular integral operators on $\mathbb{R}^{3}$ associated with Zygmund dilations by providing suitable version of regularity conditions and cancellation conditions on convolution kernels, and then show the boundedness for this class of operators on $L^{p}, 1<p<\infty$.
\end{abstract}

Keywords Multi-parameter singular integral operators · Zygmund dilations · Zygmund type cancellation

Mathematics Subject Classification 42B20 · 42B30

$\mathrm{Ji}$ Li is supported by ARC DP 160100153 and Macquarie University Seeding Grant. Chin-Cheng Lin is supported by MOST under Grant \#MOST 106-2115-M-008-004-MY3 and NCTS of Taiwan.

$\triangle \mathrm{Ji} \mathrm{Li}$

ji.li@mq.edu.au

Yongsheng Han

hanyong@mail.auburn.edu

Chin-Cheng Lin

clin@math.ncu.edu.tw

Chaoqiang Tan

cqtan@stu.edu.cn

1 Department of Mathematics, Auburn University, Auburn, AL 36849, USA

2 Department of Mathematics, Macquarie University, North Ryde, NSW 2109, Australia

3 Department of Mathematics, National Central University, Chung-Li 320, Taiwan

4 Department of Mathematics, Shantou University, Shantou 515063, Guangdong, China 


\section{Introduction and Statement of Main Results}

It is well known that Calderón and Zygmund [1] introduced certain convolution singular integral operators on $\mathbb{R}^{n}$ which generalize the Hilbert transform on $\mathbb{R}$. They proved that if $T(f)=\mathcal{K} * f$, where $\mathcal{K}$ is defined on $\mathbb{R}^{n}$ and satisfies the analogous estimates as $\frac{1}{x}$ does on $\mathbb{R}^{1}$, namely

$$
|\mathcal{K}(x)| \leq \frac{C}{|x|^{n}}, \quad|\nabla \mathcal{K}(x)| \leq \frac{C}{|x|^{n+1}},
$$

and

$$
\int_{a<|x|<b} \mathcal{K}(x) \mathrm{d} x=0 \text { for all } 0<a<b,
$$

then $T$ is bounded on $L^{p}\left(\mathbb{R}^{n}\right)$ for $1<p<\infty$. The core of this theory is that the regularity and cancellation conditions are invariant with respect to the one-parameter family of dilations on $\mathbb{R}^{n}$ defined by $\delta\left(x_{1}, x_{2}, \ldots, x_{n}\right)=\left(\delta x_{1}, \ldots, \delta x_{n}\right), \delta>0$, in the sense that the kernel $\delta^{n} \mathcal{K}(\delta x)$ satisfies the same conditions with the same bound as $\mathcal{K}(x)$.

On the other hand, the multiparameter theory of $\mathbb{R}^{n}$ began with Zygmund's study of the strong maximal function defined by

$$
\mathcal{M}_{n}(f)(x)=\sup _{R \ni x} \frac{1}{|R|} \int_{R}|f(y)| d y,
$$

where $R$ are the rectangles in $\mathbb{R}^{n}$ with sides parallel to the axes, and then continued with Marcinkiewicz's proof of his multiplier theorem. If we consider the family of product dilations defined by $\delta\left(x_{1}, x_{2}, \ldots, x_{n}\right)=\left(\delta_{1} x_{1}, \ldots, \delta_{n} x_{n}\right), \delta_{i}>0, i=1, \ldots, n$, then the strong maximal function and Marcinkiewicz's multiplier are invariant under the product dilations. The multiparameter dilations are also associated with problems in the theory of differentiation of integrals. Jensen-Marcinkiewicz-Zygmund [17] proved that the strong maximal function in $\mathbb{R}^{n}$ is bounded from the Orlicz space $L\left(1+\left(\log ^{+} L\right)^{n-1}\right)$ to weak $L^{1}$.

In [14], Fefferman and Stein generalized the singular integral operator theory to the product space. They took the space $\mathbb{R}^{n} \times \mathbb{R}^{m}$ along with the two-parameter family of dilations $(x, y) \mapsto\left(\delta_{1} x, \delta_{2} y\right),(x, y) \in \mathbb{R}^{n} \times \mathbb{R}^{m}, \delta_{1}, \delta_{2}>0$. Those operators considered in [14] generalize the double Hilbert transform on $\mathbb{R}^{2}$ given by $H(f)=$ $f * \frac{1}{x y}$ and are of the form $T(f)=\mathcal{K} * f$, where the kernel $\mathcal{K}$ is characterized by the cancellation properties

$$
\begin{aligned}
& \int_{a<|x|<b} \mathcal{K}(x, y) \mathrm{d} x=0 \text { for all } 0<a<b \text { and } y \in \mathbb{R}^{m} \\
& \int_{a<|y|<b} \mathcal{K}(x, y) d y=0 \text { for all } 0<a<b \text { and } x \in \mathbb{R}^{n}
\end{aligned}
$$


and the regularity conditions

$$
\left|\partial_{x}^{\alpha} \partial_{y}^{\beta} \mathcal{K}(x, y)\right| \leq C_{\alpha, \beta}|x|^{-n-|\alpha|}|y|^{-m-|\beta|}, \quad \alpha, \beta \geq 0 .
$$

Under the conditions (1.3)-(1.5), Fefferman and Stein proved the $L^{p}, 1<p<\infty$, boundedness of the product convolution operators $T(f)=\mathcal{K} * f$. See [14] for more details. Note that the kernel $\mathcal{K}$ satisfying the conditions (1.3)-(1.5) is invariant with respect to the product dilation in the sense that the kernel $\delta_{1}^{n} \delta_{2}^{m} \mathcal{K}\left(\delta_{1} x, \delta_{2} y\right)$ satisfies conditions (1.3)-(1.5) with the same bound. For more discussions about the multiparameter product theory, see for example [2-12,15,16,18-21,27,29] and in particular the survey article of Fefferman [12] for development in this area. For singular integrals with flag kernels, see [22,24-26].

It has been widely considered that the next simplest multiparameter group of dilations after the product multiparameter dilations is the so-called the Zygmund dilation defined on $\mathbb{R}^{3}$ by $\rho_{s, t}\left(x_{1}, x_{2}, x_{3}\right)=\left(s x_{1}, t x_{2}\right.$, st $\left.x_{3}\right)$ for $s, t>0$. Corresponding to this Zygmund dilation, one has the maximal function

$$
\mathcal{M}_{\mathfrak{z}} f(x)=\sup _{R \ni x} \frac{1}{|R|} \int_{R}|f(u)| d u,
$$

where supremum above is taken over all rectangles in $\mathbb{R}^{3}$ with sides parallel to the axes and side lengths of the form $s, t$, and $s t$. As far as $\mathcal{M}_{\mathfrak{z}}$ is concerned, Stein was the first to link the properties of maximal operators associated with Zygmund dilations to boundary value problems for Poisson integrals on symmetric spaces, such as Siegel's upper half space. We refer to the survey paper of Fefferman [10] on the future direction of research of multiparameter analysis on Zygmund dilations.

Besides $\mathcal{M}_{\mathfrak{z}}$, Ricci and Stein [28] introduced a class of singular integrals with more general dilations. One of those singular integrals is an explicit operator associated with Zygmund dilation of the form $T_{\mathfrak{z}} f=f * \mathcal{K}$, where

$$
\mathcal{K}\left(x_{1}, x_{2}, x_{3}\right)=\sum_{k, j \in \mathbb{Z}} 2^{-2(k+j)} \phi\left(\frac{x_{1}}{2^{j}}, \frac{x_{2}}{2^{k}}, \frac{x_{3}}{2^{j+k}}\right)
$$

and the function $\phi$ is supported in an unit cube in $\mathbb{R}^{3}$ with a certain amount of uniform smoothness and satisfies cancellation conditions

$$
\int_{\mathbb{R}^{2}} \phi\left(x_{1}, x_{2}, x_{3}\right) \mathrm{d} x_{1} \mathrm{~d} x_{2}=\int_{\mathbb{R}^{2}} \phi\left(x_{1}, x_{2}, x_{3}\right) \mathrm{d} x_{2} \mathrm{~d} x_{3}=\int_{\mathbb{R}^{2}} \phi\left(x_{1}, x_{2}, x_{3}\right) \mathrm{d} x_{3} \mathrm{~d} x_{1}=0 .
$$

It was shown in [28] that $T_{\mathfrak{z}}$ is bounded on $L^{p}\left(\mathbb{R}^{3}\right)$ for all $1<p<\infty$. Particularly, as mentioned in [13], the above cancellation conditions are also necessary for the boundedness of the above-mentioned operators on $L^{2}\left(\mathbb{R}^{3}\right)$. It is easy to see that if the dyadic Zygmund dilation is given by $\left(\delta_{2^{j}, 2^{k}} f\right)\left(x_{1}, x_{2}, x_{3}\right)=$ $2^{2(j+k)} f\left(2^{j} x_{1}, 2^{k} x_{2}, 2^{(j+k)} x_{3}\right)$, then we obtain that $\left(\delta_{2^{j}, 2^{k}} T_{\mathfrak{z}}(f)\right)\left(x_{1}, x_{2}, x_{3}\right)=$ $T_{\mathfrak{z}}\left(\delta_{2^{j}, 2^{k}} f\right)\left(x_{1}, x_{2}, x_{3}\right)$. This means that the operators studied by Ricci and Stein commute with Zygmund dilations of dyadic form. See [28] for more details. Fefferman 
and Pipher [13] further showed that $T_{\mathfrak{z}}$ is bounded in $L_{w}^{p}$ spaces for $1<p<\infty$ when the weight $w$ satisfies an analogous condition of Muckenhoupt associated with Zygmund dilations.

Another explicit example related to the Zygmund dilation is an operator studied by Nagel-Wainger [23], which is the singular integrals along certain surfaces in $\mathbb{R}^{3}$, defined as $T f=f * \mathcal{K}$, where

$$
\mathcal{K}\left(x_{1}, x_{2}, x_{3}\right)=\operatorname{sgn}\left(x_{1} x_{2}\right)\left\{\frac{1}{\left|x_{1}\right|^{2}\left|x_{2}\right|^{2}+x_{3}^{2}}\right\} .
$$

Motivated by these specific operators with the convolution kernel as in (1.6) and (1.7), in the current paper we introduce a class of singular integral operators associated with Zygmund dilations by providing suitable version of regularity conditions and cancellation conditions for the convolution kernel and then show the boundedness for these operators on $L^{p}, 1<p<\infty$. We also provide another versions of cancellation conditions via normalized bump functions introduced by Stein [29].

To be more precise, suppose that $\mathcal{K}\left(x_{1}, x_{2}, x_{3}\right)$ is a function defined on $\mathbb{R}^{3}$ away from the union $\left\{0, x_{2}, x_{3}\right\} \cup\left\{x_{1}, 0, x_{3}\right\} \cup\left\{x_{1}, x_{2}, 0\right\}$. For integers $\alpha, \beta$, and $\gamma$ taking only values 0 or 1 , we define

$$
\begin{aligned}
& \Delta_{x_{1}, h_{1}}^{\alpha} \mathcal{K}\left(x_{1}, x_{2}, x_{3}\right)=\alpha \mathcal{K}\left(x_{1}+h_{1}, x_{2}, x_{3}\right)-\mathcal{K}\left(x_{1}, x_{2}, x_{3}\right), \quad \alpha=0 \text { or } 1 \\
& \Delta_{x_{2}, h_{2}}^{\beta} \mathcal{K}\left(x_{1}, x_{2}, x_{3}\right)=\beta \mathcal{K}\left(x_{1}, x_{2}+h_{2}, x_{3}\right)-\mathcal{K}\left(x_{1}, x_{2}, x_{3}\right), \quad \beta=0 \text { or } 1
\end{aligned}
$$

and

$$
\Delta_{x_{3}, h_{3}}^{\gamma} \mathcal{K}\left(x_{1}, x_{2}, x_{3}\right)=\gamma \mathcal{K}\left(x_{1}, x_{2}, x_{3}+h_{3}\right)-\mathcal{K}\left(x_{1}, x_{2}, x_{3}\right), \quad \gamma=0 \text { or } 1 .
$$

For simplicity, we denote $\Delta_{x_{1}, h_{1}}=\Delta_{x_{1}, h_{1}}^{1}, \Delta_{x_{2}, h_{2}}=\Delta_{x_{2}, h_{2}}^{1}$ and $\Delta_{x_{3}, h_{3}}=\Delta_{x_{3}, h_{3}}^{1}$.

The "regularity" conditions considered in this paper are characterized by

$$
\left|\Delta_{x_{1}, h_{1}}^{\alpha} \Delta_{x_{2}, h_{2}}^{\beta} \Delta_{x_{3}, h_{3}}^{\gamma} \mathcal{K}\left(x_{1}, x_{2}, x_{3}\right)\right| \leq \frac{C\left|h_{1}\right|^{\alpha \theta_{1}}\left|h_{2}\right|^{\beta \theta_{1}}\left|h_{3}\right|^{\gamma \theta_{1}}}{\left|x_{1}\right|^{\alpha \theta_{1}+1}\left|x_{2}\right|^{\beta \theta_{1}+1}\left|x_{3}\right|^{\gamma \theta_{1}+1}\left(\left|\frac{x_{1} x_{2}}{x_{3}}\right|+\left|\frac{x_{3}}{x_{1} x_{2}}\right|\right)^{\theta_{2}}}
$$

for all $0 \leq \alpha \leq 1,0 \leq \beta+\gamma \leq 1$ or $0 \leq \alpha+\gamma \leq 1,0 \leq \beta \leq 1$, and $\left|x_{1}\right| \geq 2\left|h_{1}\right|>$ $0,\left|x_{2}\right| \geq 2\left|h_{2}\right|>0,\left|x_{3}\right| \geq 2\left|h_{3}\right|>0, h_{1}, h_{2}, h_{3} \in \mathbb{R}$ and some $0<\theta_{1} \leq 1,0<$ $\theta_{2}<1$.

Note that for any fixed non-zero two variables, say, $x_{1} \neq 0$ and $x_{2} \neq 0$, $\mathcal{K}\left(x_{1}, x_{2}, x_{3}\right)$ is an integrable function with respect to the variable $x_{3}$ and the resulting integral $\widetilde{K}\left(x_{1}, x_{2}\right)=\int_{\mathbb{R}} \mathcal{K}\left(x_{1}, x_{2}, x_{3}\right) \mathrm{d} x_{3}$, as a kernel on $\mathbb{R}^{2}$, satisfies the regularity conditions of the classical product kernel on $\mathbb{R}^{2}$ as studied by Fefferman and Stein in [14]. These facts, as mentioned above, can also be easily checked for singular integral operator $T_{\mathfrak{z}}$. 
In this paper, we consider three kinds of cancellation conditions. The first one is

$$
\left|\int_{\delta_{1} \leq\left|x_{1}\right| \leq r_{1}} \int_{\delta_{2} \leq\left|x_{2}\right| \leq r_{2}} \int_{\delta_{3} \leq\left|x_{3}\right| \leq r_{3}} \mathcal{K}\left(x_{1}, x_{2}, x_{3}\right) \mathrm{d} x_{1} \mathrm{~d} x_{2} \mathrm{~d} x_{3}\right| \leq C
$$

uniformly for all $\delta_{1}, \delta_{2}, \delta_{3}, r_{1}, r_{2}, r_{3}>0$;

$$
\left|\int_{\delta_{1} \leq\left|x_{1}\right| \leq r_{1}} \int_{\delta_{2} \leq\left|x_{2}\right| \leq r_{2}} \Delta_{x_{3}, h_{3}}^{\gamma} \mathcal{K}\left(x_{1}, x_{2}, x_{3}\right) \mathrm{d} x_{1} \mathrm{~d} x_{2}\right| \leq \frac{C\left|h_{3}\right|^{\gamma \theta_{1}}}{\left|x_{3}\right|^{\gamma \theta_{1}+1}}
$$

uniformly for all $\delta_{1}, \delta_{2}, r_{1}, r_{2}>0,\left|x_{3}\right| \geq 2\left|h_{3}\right|>0$ and $0 \leq \gamma \leq 1$;

$$
\left|\int_{\delta_{2} \leq\left|x_{2}\right| \leq r_{2}} \int_{\delta_{3} \leq\left|x_{3}\right| \leq r_{3}} \Delta_{x_{1}, h_{1}}^{\alpha} \mathcal{K}\left(x_{1}, x_{2}, x_{3}\right) \mathrm{d} x_{2} \mathrm{~d} x_{3}\right| \leq \frac{C\left|h_{1}\right|^{\alpha \theta_{1}}}{\left|x_{1}\right|^{\alpha \theta_{1}+1}}
$$

uniformly for all $\delta_{2}, \delta_{3}, r_{2}, r_{3}>0,\left|x_{1}\right| \geq 2\left|h_{1}\right|>0$ and $0 \leq \alpha \leq 1$;

$$
\left|\int_{\delta_{1} \leq\left|x_{1}\right| \leq r_{1}} \int_{\delta_{3} \leq\left|x_{3}\right| \leq r_{3}} \Delta_{x_{2}, h_{2}}^{\beta} \mathcal{K}\left(x_{1}, x_{2}, x_{3}\right) \mathrm{d} x_{3} \mathrm{~d} x_{1}\right| \leq \frac{C\left|h_{2}\right|^{\beta \theta_{1}}}{\left|x_{2}\right|^{\beta \theta_{1}+1}}
$$

uniformly for all $\delta_{1}, \delta_{3}, r_{1}, r_{3}>0,\left|x_{2}\right| \geq 2\left|h_{2}\right|>0$ and $0 \leq \beta \leq 1$.

The regularity conditions (RR) and the cancellation conditions (C1.a)-(C1.d) imply the following $L^{2}$ boundedness.

Theorem 1.1 Suppose that $\mathcal{K}$ is a function defined on $\mathbb{R}^{3}$ and satisfies the conditions (RR) and (C1.a)-(C1.d). Set $\mathcal{K}_{\epsilon}^{N}\left(x_{1}, x_{2}, x_{3}\right)=\mathcal{K}\left(x_{1}, x_{2}, x_{3}\right)$ if $\epsilon_{1} \leq\left|x_{1}\right| \leq N_{1}, \epsilon_{2} \leq$ $\left|x_{2}\right| \leq N_{2}$ and $\epsilon_{3} \leq\left|x_{3}\right| \leq N_{3}$ and $\mathcal{K}_{\epsilon}^{N}\left(x_{1}, x_{2}, x_{3}\right)=0$ otherwise, where $\epsilon=$ $\left(\epsilon_{1}, \epsilon_{2}, \epsilon_{3}\right)$ and $N=\left(N_{1}, N_{2}, N_{3}\right)$ for all $0<\epsilon_{1} \leq N_{1}<\infty, \epsilon_{2} \leq N_{2}<\infty$, and $\epsilon_{3} \leq N_{3}<\infty$. Then, the operator $\mathcal{K}_{\epsilon}^{N} * f$ is bounded on $L^{2}\left(\mathbb{R}^{3}\right)$ and moreover,

$$
\left\|\mathcal{K}_{\epsilon}^{N} * f\right\|_{L^{2}\left(\mathbb{R}^{3}\right)} \leq A\|f\|_{L^{2}\left(\mathbb{R}^{3}\right)}
$$

where the constant A depends only on the constant $C$ but not on $\epsilon_{1}, \epsilon_{2}, \epsilon_{3}, N_{1}, N_{2}$ and $N_{3}$.

From Theorem 1.1, we will deduce the existence of the corresponding singular integrals in the $L^{2}$ norm as a limit of the truncated integrals.

Corollary 1.2 Suppose that $\mathcal{K}$ is a function defined on $\mathbb{R}^{3}$ and satisfies the conditions (RR), (C1.a)-(C1.d) and, in addition, the three integrals

$$
\begin{aligned}
& \int_{\left|x_{3}\right| \leq 1} \int_{\left|x_{2}\right| \leq 1} \int_{\left|x_{1}\right| \leq 1} K_{\epsilon}^{N}\left(x_{1}, x_{2}, x_{3}\right) \mathrm{d} x_{1} \mathrm{~d} x_{2} \mathrm{~d} x_{3}, \\
& \int_{\left|x_{3}\right| \leq 1} \int_{\left|x_{2}\right| \leq 1} \mathcal{K}_{\epsilon}^{N}\left(x_{1}, x_{2}, x_{3}\right) \mathrm{d} x_{2} \mathrm{~d} x_{3}
\end{aligned}
$$




$$
\int_{\left|x_{3}\right| \leq 1} \int_{\left|x_{1}\right| \leq 1} \mathcal{K}_{\epsilon}^{N}\left(x_{1}, x_{2}, x_{3}\right) \mathrm{d} x_{1} \mathrm{~d} x_{3}
$$

converge almost everywhere as $\epsilon_{1}, \epsilon_{2}, \epsilon_{3} \rightarrow 0$ and $N_{1}, N_{2}, N_{3} \rightarrow \infty$. Then the limit $\lim _{\epsilon_{1}, \epsilon_{2}, \epsilon_{3} \rightarrow 0} \mathcal{K}_{\epsilon}^{N} * f=\mathcal{K} * f$ exists in the $L^{2}\left(\mathbb{R}^{3}\right)$ norm. Moreover, $N_{1}, N_{2}, N_{3} \rightarrow \infty$

$$
\|\mathcal{K} * f\|_{L^{2}\left(\mathbb{R}^{3}\right)} \leq A\|f\|_{L^{2}\left(\mathbb{R}^{3}\right)}
$$

with the constant A depending only on the constant $C$.

We remark in advance that the proof of Corollary 1.2 indeed implies that $\lim \epsilon_{1}, \epsilon_{2}, \epsilon_{3} \rightarrow 0 \quad \mathcal{K}_{\epsilon}^{N} * f$ exists in the $L^{p}, 1<p<\infty$, norm for smooth functions $f$ $N_{1}, N_{2}, N_{3} \rightarrow \infty$

having compact support. This fact leads to the study of the $L^{p}, p \neq 2$, boundedness of the operator $\mathcal{K} * f$. For this purpose, we need the second kind of the cancellation conditions which are somewhat stronger than the first ones. They are given by

$$
\left|\int_{\delta_{3} \leq\left|x_{3}\right| \leq r_{3}} \int_{\delta_{2} \leq\left|x_{2}\right| \leq r_{2}} \int_{\delta_{1} \leq\left|x_{1}\right| \leq r_{1}} \mathcal{K}\left(x_{1}, x_{2}, x_{3}\right) \mathrm{d} x_{1} \mathrm{~d} x_{2} \mathrm{~d} x_{3}\right| \leq C
$$

uniformly for all $\delta_{1}, \delta_{2}, \delta_{3}, r_{1}, r_{2}, r_{3}>0$;

$$
\begin{aligned}
& \left|\int_{\delta_{1} \leq\left|x_{1}\right| \leq r_{1}} \Delta_{x_{2}, h_{2}}^{\beta} \Delta_{x_{3}, h_{3}}^{\gamma} \mathcal{K}\left(x_{1}, x_{2}, x_{3}\right) \mathrm{d} x_{1}\right| \\
& \quad \leq \frac{C\left|h_{2}\right|^{\beta \theta_{1}}\left|h_{3}\right|^{\gamma \theta_{1}}}{\left|x_{2}\right|^{\beta \theta_{1}+1}\left|x_{3}\right|^{\gamma \theta_{1}+1}}\left(\frac{1}{\left(\left|\frac{r_{1} x_{2}}{x_{3}}\right|+\left|\frac{x_{3}}{r_{1} x_{2}}\right|\right)^{\theta_{2}}}+\frac{1}{\left(\left|\frac{\delta_{1} x_{2}}{x_{3}}\right|+\left|\frac{x_{3}}{\delta_{1} x_{2}}\right|\right)^{\theta_{2}}}\right)
\end{aligned}
$$

for all $\delta_{1}, r_{1}>0,0 \leq \beta+\gamma \leq 1,\left|x_{2}\right| \geq 2\left|h_{2}\right|>0,|z| \geq 2\left|h_{3}\right|>0$;

$$
\left|\int_{\delta_{3} \leq\left|x_{3}\right| \leq r_{3}} \int_{\delta_{2} \leq\left|x_{2}\right| \leq r_{2}} \Delta_{x_{1}, h_{1}}^{\alpha} \mathcal{K}\left(x_{1}, x_{2}, x_{3}\right) \mathrm{d} x_{2} \mathrm{~d} x_{3}\right| \leq \frac{C\left|h_{1}\right|^{\alpha \theta_{1}}}{\left|x_{1}\right|^{\alpha \theta_{1}+1}}
$$

uniformly for all $\delta_{2}, \delta_{3}, r_{2}, r_{3}>0,\left|x_{1}\right| \geq 2\left|h_{1}\right|>0$ and $0 \leq \alpha \leq 1$. Or

$$
\left|\int_{\delta_{3} \leq\left|x_{3}\right| \leq r_{3}} \int_{\delta_{2} \leq\left|x_{2}\right| \leq r_{2}} \int_{\delta_{1} \leq\left|x_{1}\right| \leq r_{1}} \mathcal{K}\left(x_{1}, x_{2}, x_{3}\right) \mathrm{d} x_{1} \mathrm{~d} x_{2} \mathrm{~d} x_{3}\right| \leq C
$$

uniformly for all $\delta_{1}, \delta_{2}, \delta_{3}, r_{1}, r_{2}, r_{3}>0$;

$$
\begin{aligned}
& \left|\int_{\delta_{2} \leq\left|x_{2}\right| \leq r_{2}} \Delta_{x_{1}, h_{1}}^{\alpha} \Delta_{x_{3}, h_{3}}^{\gamma} \mathcal{K}\left(x_{1}, x_{2}, x_{3}\right) \mathrm{d} x_{2}\right| \\
& \quad \leq \frac{C\left|h_{1}\right|^{\alpha \theta_{1}}\left|h_{3}\right|^{\gamma \theta_{1}}}{\left|x_{1}\right|^{\alpha \theta_{1}+1}\left|x_{3}\right|^{\gamma \theta_{1}}}\left(\frac{1}{\left(\left|\frac{r_{2} x_{1}}{x_{3}}\right|+\left|\frac{x_{3}}{r_{2} x_{1}}\right|\right)^{\theta_{2}}}+\frac{1}{\left(\left|\frac{\delta_{2} x_{1}}{x_{3}}\right|+\left|\frac{x_{3}}{\delta_{2} x_{1}}\right|\right)^{\theta_{2}}}\right)
\end{aligned}
$$


for all $\delta_{2}, r_{2}>0,0 \leq \alpha+\gamma \leq 1,\left|x_{1}\right| \geq 2\left|h_{1}\right|>0$ and $\left|x_{3}\right| \geq 2\left|h_{3}\right|>0$;

$$
\left|\int_{\delta_{3} \leq\left|x_{3}\right| \leq r_{3}} \int_{\delta_{1} \leq\left|x_{1}\right| \leq r_{1}} \Delta_{x_{2}, h_{2}}^{\beta} \mathcal{K}\left(x_{1}, x_{2}, x_{3}\right) \mathrm{d} x_{1} \mathrm{~d} x_{3}\right| \leq \frac{C\left|h_{2}\right|^{\beta \theta_{1}}}{\left|x_{2}\right|^{\beta \theta_{1}+1}}
$$

uniformly for all $\delta_{1}, \delta_{3}, r_{1}, r_{3}>0,\left|x_{2}\right| \geq 2\left|h_{2}\right|>0$ and $0 \leq \beta \leq 1$.

We would like to point out that the condition (C2.b) implies (C1.b) and (C1.d) while $\left(\mathrm{C} 2^{\prime} . \mathrm{b}\right)$ implies $(\mathrm{C} 1 . \mathrm{b})$ and $(\mathrm{C} 1 . \mathrm{c})$, and all the above regularity and cancellation conditions are invariant with respect to the Zygmund dilation in the sense that the kernel $\delta_{1}^{2} \delta_{2}^{2} \mathcal{K}\left(\delta_{1} x_{1}, \delta_{2} x_{2}, \delta_{1} \delta_{2} x_{3}\right)$ satisfies the same conditions with the exactly same bounds as $\mathcal{K}\left(x_{1}, x_{2}, x_{3}\right)$.

The $L^{p}$ estimate then is given by the following

Theorem 1.3 Suppose that $\mathcal{K}$ is a function defined on $\mathbb{R}^{3}$ and satisfies the conditions (RR) and (C2.a)-(C2.c) (or (RR), (C2'.a)-(C2'.c)) and in addition the three integrals

$$
\begin{aligned}
& \int_{\left|x_{3}\right| \leq 1} \int_{\left|x_{2}\right| \leq 1} \int_{\left|x_{1}\right| \leq 1} K_{\epsilon}^{N}\left(x_{1}, x_{2}, x_{3}\right) \mathrm{d} x_{1} \mathrm{~d} x_{2} \mathrm{~d} x_{3} \\
& \int_{\left|x_{3}\right| \leq 1} \int_{\left|x_{2}\right| \leq 1} \mathcal{K}_{\epsilon}^{N}\left(x_{1}, x_{2}, x_{3}\right) \mathrm{d} x_{2} \mathrm{~d} x_{3} \\
& \int_{\left|x_{3}\right| \leq 1} \int_{\left|x_{1}\right| \leq 1} \mathcal{K}_{\epsilon}^{N}\left(x_{1}, x_{2}, x_{3}\right) \mathrm{d} x_{1} d x_{3}
\end{aligned}
$$

converge almost everywhere as $\epsilon_{1}, \epsilon_{2}, \epsilon_{3} \rightarrow 0$ and $N_{1}, N_{2}, N_{3} \rightarrow \infty$. Then the operator

$$
\mathcal{K} * f:=\lim _{\substack{\epsilon_{1}, \epsilon_{2}, \epsilon_{3} \rightarrow 0 \\ N_{1}, N_{2}, N_{3} \rightarrow \infty}} \mathcal{K}_{\epsilon}^{N} * f
$$

defined initially on $L^{2} \cap L^{p}, 1<p<\infty$, extends to a bounded operator on $L^{p}\left(\mathbb{R}^{3}\right)$ and moreover,

$$
\|\mathcal{K} * f\|_{L^{p}\left(\mathbb{R}^{3}\right)} \leq A\|f\|_{L^{p}\left(\mathbb{R}^{3}\right)}
$$

with the constant A depending only on the constant $C$.

In many applications, singular integral operators are of the form $\mathcal{K} * f$ where $\mathcal{K}$ is a distribution that equals a function $\mathcal{K}$ on $\mathbb{R}^{3}$ away from the union $\left\{0, x_{2}, x_{3}\right\} \cup$ $\left\{x_{1}, 0, x_{3}\right\} \cup\left\{x_{1}, x_{2}, 0\right\}$ and satisfy certain regularity and cancellation conditions. For this purpose, we begin with recalling the bump functions introduced by Stein in [29]. A normalized bump function (n.b.f.) is a smooth function $\phi$ supported on the unit ball and is bounded by a fixed constant together with its gradient. The third kind of the cancellation conditions considered in this paper is characterized by

$$
\left|\iiint \mathcal{K}\left(x_{1}, x_{2}, x_{3}\right) \phi\left(R_{1} x_{1}, R_{2} x_{2}, R_{1} R_{2} x_{3}\right) \mathrm{d} x_{1} \mathrm{~d} x_{2} \mathrm{~d} x_{3}\right| \leq C
$$


for every n.b.f. $\phi$ on $\mathbb{R}^{3}$ and all $R_{1}, R_{2}>0$;

$$
\left|\int \Delta_{x_{2}, h_{2}}^{\beta} \Delta_{x_{3}, h_{3}}^{\gamma} \mathcal{K}\left(x_{1}, x_{2}, x_{3}\right) \phi\left(R x_{1}\right) \mathrm{d} x_{1}\right| \leq \frac{C\left|h_{2}\right|^{\beta \theta_{1}}\left|h_{3}\right|^{\gamma \theta_{1}}}{\left|x_{2}\right|^{\beta \theta_{1}+1}\left|x_{3}\right|^{\gamma \theta_{1}+1}\left(\left|\frac{R x_{3}}{x_{2}}\right|+\left|\frac{x_{2}}{R x_{3}}\right|\right)^{\theta_{2}}}
$$

for all $0 \leq \beta+\gamma \leq 1$, every n.b.f. $\phi$ on $\mathbb{R},\left|x_{2}\right| \geq 2\left|h_{2}\right|>0,\left|x_{3}\right| \geq 2\left|h_{3}\right|>0$ and all $R>0$;

$$
\left|\iint \Delta_{x_{1}, h_{1}}^{\alpha} \mathcal{K}\left(x_{1}, x_{2}, x_{3}\right) \phi\left(R_{1} x_{2}, R_{2} x_{3}\right) \mathrm{d} x_{2} \mathrm{~d} x_{3}\right| \leq \frac{C\left|h_{1}\right|^{\alpha \theta_{1}}}{\left|x_{1}\right|^{\alpha \theta_{1}+1}}
$$

for all $0 \leq \alpha \leq 1$, every n.b.f. $\phi$ on $\mathbb{R}^{2},\left|x_{1}\right| \geq 2\left|h_{1}\right|>0$ and all $R_{1}, R_{2}>0$. Or

$$
\left|\iiint \mathcal{K}\left(x_{1}, x_{2}, x_{3}\right) \phi\left(R_{1} x_{1}, R_{2} x_{2}, R_{1} R_{2} x_{3}\right) \mathrm{d} x_{1} \mathrm{~d} x_{2} \mathrm{~d} x_{3}\right| \leq C
$$

for every n.b.f. $\phi$ on $\mathbb{R}^{3}$ and all $R_{1}, R_{2}>0$;

$$
\left|\int \Delta_{x_{1}, h_{1}}^{\alpha} \Delta_{x_{3}, h_{3}}^{\gamma} \mathcal{K}\left(x_{1}, x_{2}, x_{3}\right) \phi\left(R x_{2}\right) \mathrm{d} x_{2}\right| \leq \frac{C\left|h_{1}\right|^{\alpha \theta_{1}}\left|h_{3}\right|^{\gamma \theta_{1}}}{\left|x_{1}\right|^{\alpha \theta_{1}+1}\left|x_{3}\right|^{\gamma \theta_{1}+1}\left(\left|\frac{R x_{3}}{x_{1}}\right|+\left|\frac{x_{1}}{R x_{3}}\right|\right)^{\theta_{2}}}
$$

for all $0 \leq \alpha+\gamma \leq 1$, every n.b.f. $\phi$ on $\mathbb{R},\left|x_{1}\right| \geq 2\left|h_{1}\right|>0,\left|x_{3}\right| \geq 2\left|h_{3}\right|>0$ and all $R>0$;

$$
\left|\iint \Delta_{x_{2}, h_{2}}^{\beta} \mathcal{K}\left(x_{1}, x_{2}, x_{3}\right) \phi\left(R_{1} x_{1}, R_{2} x_{3}\right) \mathrm{d} x_{1} \mathrm{~d} x_{3}\right| \leq \frac{C\left|h_{2}\right|^{\beta \theta_{1}}}{\left|x_{2}\right|^{\beta \theta_{1}+1}}
$$

for all $0 \leq \beta \leq 1$, every n.b.f. $\phi$ on $\mathbb{R}^{2},\left|x_{2}\right| \geq 2\left|h_{2}\right|>0$ and all $R_{1}, R_{2}>0$.

Theorem 1.4 Let all the notation be the same as above.

(a) Suppose that $\mathcal{K}$ is a distribution that equals a function on $\mathbb{R}^{3}$ away from the union $\left\{0, x_{2}, x_{3}\right\} \cup\left\{x_{1}, 0, x_{3}\right\} \cup\left\{x_{1}, x_{2}, 0\right\}$ and satisfies conditions (RR) and (C3.a)-(C3.c) (or (RR), (C3'.a)-(C3'.c)). Then, the operator $\mathcal{K} * f$ is bounded on $L^{p}\left(\mathbb{R}^{3}\right), 1<p<\infty$; moreover,

$$
\|\mathcal{K} * f\|_{L^{p}\left(\mathbb{R}^{3}\right)} \leq A\|f\|_{L^{p}\left(\mathbb{R}^{3}\right)}
$$

with the constant A depending only on the constant $C$.

(b) Suppose that $\mathcal{K}$ is a distribution that equals a function on $\mathbb{R}^{3}$ away from the union $\left\{0, x_{2}, x_{3}\right\} \cup\left\{x_{1}, 0, x_{3}\right\} \cup\left\{x_{1}, x_{2}, 0\right\}$ and satisfies conditions (RR) and (C2.a)-(C2.c) (or (RR), (C2' .a)-(C2'.c)). Then, the operator $\mathcal{K} * f$ is bounded on $L^{p}\left(\mathbb{R}^{3}\right), 1<p<\infty$, and 


$$
\|\mathcal{K} * f\|_{L^{p}\left(\mathbb{R}^{3}\right)} \leq A\|f\|_{L^{p}\left(\mathbb{R}^{3}\right)}
$$

with the constant A depending only on the constant $C$.

Remark 1.5 We would like to point out that all regularity and cancellation conditions given above are invariant with respect to Zygmund dilations. Moreover, The boundedness results in this paper can be extended to higher dimensions. The consideration of regularity and cancellation conditions in this paper leads naturally to the study of nonconvolution singular integral operators which are associated with Zygmund dilations. We will discuss all these topics in the forthcoming works.

Theorem 1.6 Let all the notation be the same as above.

(i) The singular integral operator $T_{\mathfrak{z}}$ given in [28] with the convolution kernel as in (1.6) can be written as $T_{\mathfrak{z}}=T_{\mathfrak{z}, 1}+T_{\mathfrak{z}, 2}$, where $T_{\mathfrak{z}, i}$ is a singular integral operator with convolution kernel $\mathcal{K}_{i}, i=1,2 . \mathcal{K}_{1}$ satisfies conditions $(\mathrm{RR})$ and (C2.a)-(C2.c), and $\mathcal{K}_{2}$ satisfies conditions $\left.(\mathrm{RR}),\left(\mathrm{C} 2^{\prime} . \mathrm{a}\right)-\left(\mathrm{C} 2^{\prime} . \mathrm{c}\right)\right)$.

(ii) The singular integral operator $T$ given in [23] with the convolution kernel as in (1.7) satisfy conditions (RR) and (C2.a)-(C2.c).

Remark 1.7 Note that for the operator $T$ from [23] with the convolution kernel as in (1.7), only the $L^{2}$ boundedness is proved. Hence, as a consequence of Theorem 1.3, $T$ is bounded on $L^{p}\left(\mathbb{R}^{3}\right)$ for all $1<p<\infty$.

The organization of this paper is as follows: In the next section, we will show the $L^{2}$ boundedness for singular integral operators associated with Zygmund dilations, namely Theorem 1.1 and Corollary 1.2. The $L^{p}$ boundedness, namely Theorems 1.3 and 1.4, will be proved in Sect. 3. In the last section, we prove Theorem 1.6.

\section{$2 L^{2}$ Boundedness}

The main task of this section is to provide proofs of Theorem 1.1 and Corollary 1.2. Before proving Theorem 1.1, we first prove the following simple result which will be used frequently below.

Lemma 2.1 For any $f(x) \in L_{\text {loc }}^{1}(\mathbb{R})$ and $N>8$, we have

$$
\left|\int_{8 \leq|x| \leq N} f(x) e^{-i x} \mathrm{~d} x\right| \leq \frac{1}{2} \int_{E_{N}}|f(x)| \mathrm{d} x+\frac{1}{2} \int_{8 \leq|x| \leq N}|f(x)-f(x+\pi)| \mathrm{d} x,
$$

where $E_{N}=\{x: 4 \leq|x| \leq 12\} \cup\{x: N-\pi \leq|x| \leq N+\pi\}$.

Proof We write

$$
\begin{aligned}
\int_{8 \leq|x| \leq N} f(x) e^{-i x} \mathrm{~d} x & =\int_{8 \leq|x+\pi| \leq N} f(x+\pi) e^{-i(x+\pi)} \mathrm{d} x \\
& =-\int_{8 \leq|x+\pi| \leq N} f(x+\pi) e^{-i x} \mathrm{~d} x .
\end{aligned}
$$


Therefore,

$$
\begin{aligned}
\left|\int_{8 \leq|x| \leq N} f(x) e^{-i x} \mathrm{~d} x\right|= & \frac{1}{2}\left|\int_{8 \leq|x| \leq N} f(x) e^{-i x} \mathrm{~d} x-\int_{8 \leq|x+\pi| \leq N} f(x+\pi) e^{-i x} \mathrm{~d} x\right| \\
\leq & \frac{1}{2}\left|\int_{8 \leq|x| \leq N}(f(x)-f(x+\pi)) e^{-i x} \mathrm{~d} x\right| \\
& +\frac{1}{2}\left|\int_{\{x: 8 \leq|x| \leq N\} \backslash\{x: 8 \leq|x+\pi| \leq N\}} f(x+\pi) e^{-i x} \mathrm{~d} x\right| \\
& +\frac{1}{2}\left|\int_{\{x: 8 \leq|x+\pi| \leq N\} \backslash\{x: 8 \leq|x| \leq N\}} f(x+\pi) e^{-i x} \mathrm{~d} x\right| \\
= & \frac{1}{2}\left|\int_{8 \leq|x| \leq N}(f(x)-f(x+\pi)) e^{-i x} \mathrm{~d} x\right| \\
& +\frac{1}{2}\left|\int_{\{x: 8 \leq|x-\pi| \leq N\} \backslash\{x: 8 \leq|x| \leq N\}} f(x) e^{-i x} \mathrm{~d} x\right| \\
& +\frac{1}{2}\left|\int_{\{x: 8 \leq|x| \leq N\} \backslash\{x: 8 \leq|x-\pi| \leq N\}} f(x) e^{-i x} \mathrm{~d} x\right| \\
\leq & \frac{1}{2} \int_{8 \leq|x| \leq N}|f(x)-f(x+\pi)| \mathrm{d} x+\frac{1}{2} \int_{E_{N}}|f(x)| \mathrm{d} x
\end{aligned}
$$

and Lemma 2.1 follows.

We now prove Theorem 1.1.

Proof of Theorem 1.1 By the Plancherel theorem, the $L^{2}$ boundedness of $\mathcal{K}_{\epsilon}^{N} * f$ is equivalent to $\left|\widehat{\mathcal{K}_{\epsilon}^{N}}(\chi, \eta, \xi)\right| \leq A$, where $\widehat{\mathcal{K}_{\epsilon}^{N}}$ is the Fourier transform of $\mathcal{K}_{\epsilon}^{N}$ and $A$ is the constant depending only on the constant $C$ but not on $\epsilon=\left(\epsilon_{1}, \epsilon_{2}, \epsilon_{3}\right)$ and $N=\left(N_{1}, N_{2}, N_{3}\right)$. To obtain such an estimate, we may assume that $\chi$ and $\eta$ are both positive. Note that,

$$
\begin{gathered}
\widehat{\mathcal{K}_{\epsilon}^{N}}(\chi, \eta, \xi)= \\
\int_{\epsilon_{3} \leq\left|x_{3}\right| \leq N_{3}} \int_{\epsilon_{2} \leq\left|x_{2}\right| \leq N_{1}} \int_{\epsilon_{1} \leq\left|x_{1}\right| \leq N_{1}} \mathcal{K}\left(x_{1}, x_{2}, x_{3}\right) e^{-i x \chi} e^{-i y \eta} e^{-i z \xi} \mathrm{d} x_{1} \mathrm{~d} x_{2} \mathrm{~d} x_{3} \\
=\int_{\frac{\epsilon_{3}}{\chi \eta} \leq\left|x_{3}\right| \leq \frac{N_{3}}{\chi \eta}} \int_{\frac{\epsilon_{2}}{\eta} \leq\left|x_{2}\right| \leq \frac{N_{2}}{\eta}} \int_{\frac{\epsilon_{1}}{\chi} \leq\left|x_{1}\right| \leq \frac{N_{1}}{\chi}} \frac{1}{\chi^{2} \eta^{2}} \mathcal{K}\left(\frac{x_{1}}{\chi}, \frac{x_{2}}{\eta}, \frac{x_{3}}{\chi \eta}\right) \\
\cdot e^{-i x_{1}} e^{-i x_{2}} e^{-i x_{3} \xi /(\chi \eta)} \mathrm{d} x_{1} \mathrm{~d} x_{2} \mathrm{~d} x_{3} .
\end{gathered}
$$

As we remarked above that the assumptions on $\mathcal{K}$ are invariant in the sense that $\delta_{1}^{2} \delta_{2}^{2} \mathcal{K}\left(\delta_{1} x_{1}, \delta_{2} x_{2}, \delta_{1} \delta_{2} x_{3}\right)$ satisfies the same assumptions as $\mathcal{K}$ with the same constant $C$, independent of $\delta_{1}, \delta_{2}>0$. Thus $\frac{1}{\chi^{2} \eta^{2}} \mathcal{K}\left(\frac{x_{1}}{\chi}, \frac{x_{2}}{\eta}, \frac{x_{3}}{\chi \eta}\right)$ satisfies all conditions (RR) and (C1.a)-(C1.d) with the same bounds uniformly for $\chi, \eta$. Therefore, it suffices to show that $\widehat{\mathcal{K}_{\epsilon}^{N}}(1,1, \xi)$ is a bounded function uniformly for $0<\epsilon_{1}, \epsilon_{2}, \epsilon_{3}, N_{1}, N_{2}, N_{3}<\infty$. To do this, for simplicity, we set $\epsilon_{4}=\epsilon_{3}|\xi|$ and $N_{4}=N_{3}|\xi|$. Without loss of generality, we may assume that $\epsilon_{1}, \epsilon_{2}, \epsilon_{4} \leq 8 \leq N_{1}, N_{2}, N_{4}$ since all other cases can be written as a finite linear combination of these cases and can be handled similarly. 
The bound of $\widehat{\mathcal{K}_{\epsilon}^{N}}(1,1, \xi)$ follows from the regularity and cancellation conditions on $\mathcal{K}$. More precisely, we write

$$
\begin{aligned}
& \widehat{\mathcal{K}_{\epsilon}^{N}}(1,1, \xi)=\int_{\epsilon_{3} \leq\left|x_{3}\right| \leq N_{3}} \int_{\epsilon_{2} \leq\left|x_{2}\right| \leq N_{2}} \int_{\epsilon_{1} \leq\left|x_{1}\right| \leq N_{1}} \\
& \mathcal{K}\left(x_{1}, x_{2}, x_{3}\right) e^{-i x_{1}} e^{-i x_{2}} e^{-i x_{3} \xi} \mathrm{d} x_{1} \mathrm{~d} x_{2} \mathrm{~d} x_{3} \\
& =\int_{\epsilon_{4} \leq\left|x_{3}\right| \leq N_{4}} \int_{\int_{\epsilon_{2} \leq\left|x_{2}\right| \leq N_{2}}} \int_{\epsilon_{1} \leq\left|x_{1}\right| \leq N_{1}} \frac{1}{\xi} \mathcal{K}\left(x_{1}, x_{2}, \frac{x_{3}}{\xi}\right) \\
& \times e^{-i x_{1}} e^{-i x_{2}} e^{-i x_{3}} \mathrm{~d} x_{1} \mathrm{~d} x_{2} \mathrm{~d} x_{3}:=I+I I \text {, }
\end{aligned}
$$

where $I$ is the result of integrating over the set $\left\{8 \leq\left|x_{1}\right| \leq N_{1}, \epsilon_{2} \leq\left|x_{2}\right| \leq N_{1}, \epsilon_{4} \leq\right.$ $\left.\left|x_{3}\right| \leq N_{4}\right\}$ and $I I$ over the set $\left\{\epsilon_{1} \leq\left|x_{1}\right|<8, \epsilon_{2} \leq\left|x_{2}\right| \leq N_{1}, \epsilon_{4} \leq\left|x_{3}\right| \leq N_{4}\right\}$.

For term $I$, using Lemma 2.1 with $f\left(x_{1}\right)=\int_{\epsilon_{2} \leq\left|x_{2}\right| \leq N_{2}} \int_{\epsilon_{4} \leq\left|x_{3}\right| \leq N_{4}} \frac{1}{\xi} \mathcal{K}\left(x_{1}, x_{2}, \frac{x_{3}}{\xi}\right)$ $e^{-i x_{2}} \cdot e^{-i x_{3}} \mathrm{~d} x_{3} \mathrm{~d} x_{2}$, we obtain

$$
\begin{aligned}
|I| \lesssim & \int_{E_{N_{1}}}\left|\int_{\epsilon_{2} \leq\left|x_{2}\right| \leq N_{2}} \int_{\epsilon_{4} \leq\left|x_{3}\right| \leq N_{4}} \frac{1}{\xi} \mathcal{K}\left(x_{1}, x_{2}, \frac{x_{3}}{\xi}\right) e^{-i x_{2}} e^{-i x_{3}} \mathrm{~d} x_{3} \mathrm{~d} x_{2}\right| \mathrm{d} x_{1} \\
& +\int_{8 \leq\left|x_{1}\right| \leq N_{1}} \mid \int_{\epsilon_{2} \leq\left|x_{2}\right| \leq N_{2}} \int_{\epsilon_{4} \leq\left|x_{3}\right| \leq N_{4}} \Delta_{x_{1}, \pi}\left(\frac{1}{\xi} \mathcal{K}\left(x_{1}, x_{2}, \frac{x_{3}}{\xi}\right)\right) \\
& \times e^{-i x_{2}} e^{-i x_{3}} \mathrm{~d} x_{3} \mathrm{~d} x_{2} \mid \mathrm{d} x_{1} \\
:= & I_{1}+I_{2} .
\end{aligned}
$$

We write $I_{1}$ by

$$
\begin{aligned}
I_{1} \leq & \int_{E_{N_{1}}}\left|\int_{8 \leq\left|x_{2}\right| \leq N_{2}} \int_{\epsilon_{4} \leq\left|x_{3}\right| \leq N_{4}} \frac{1}{\xi} \mathcal{K}\left(x_{1}, x_{2}, \frac{x_{3}}{\xi}\right) e^{-i x_{2}} e^{-i x_{3}} \mathrm{~d} x_{3} \mathrm{~d} x_{2}\right| \mathrm{d} x_{1} \\
& +\int_{E_{N_{1}}}\left|\int_{\epsilon_{2} \leq\left|x_{2}\right| \leq 8} \int_{\epsilon_{4} \leq\left|x_{3}\right| \leq N_{4}} \frac{1}{\xi} \mathcal{K}\left(x_{1}, x_{2}, \frac{x_{3}}{\xi}\right) e^{-i x_{2}} e^{-i x_{3}} \mathrm{~d} x_{3} \mathrm{~d} x_{2}\right| \mathrm{d} x_{1} \\
:= & I_{1,1}+I_{1,2} .
\end{aligned}
$$

To estimate term $I_{1,1}$, using Lemma 2.1 with $f\left(x_{2}\right)=\int_{\epsilon_{4} \leq\left|x_{3}\right| \leq N_{4}} \frac{1}{\xi} \mathcal{K}\left(x_{1}, x_{2}, \frac{x_{3}}{\xi}\right)$ $e^{-i x_{3}} \mathrm{~d} x_{3}$ we get

$$
\begin{aligned}
\left|I_{1,1}\right| \lesssim & \int_{E_{N_{1}}} \int_{E_{N_{2}}}\left|\int_{\epsilon_{4} \leq\left|x_{3}\right| \leq N_{4}} \frac{1}{\xi} \mathcal{K}\left(x_{1}, x_{2}, \frac{x_{3}}{\xi}\right) e^{-i x_{3}} \mathrm{~d} x_{3}\right| \mathrm{d} x_{2} \mathrm{~d} x_{1} \\
& +\int_{E_{N_{1}}} \int_{8 \leq\left|x_{2}\right| \leq N_{2}}\left|\int_{\epsilon_{4} \leq\left|x_{3}\right| \leq N_{4}} \Delta_{x_{2}, \pi}\left(\frac{1}{\xi} \mathcal{K}\left(x_{1}, x_{2}, \frac{x_{3}}{\xi}\right)\right) e^{-i x_{3}} \mathrm{~d} x_{3}\right| \mathrm{d} x_{2} \mathrm{~d} x_{1} \\
& \lesssim \int_{E_{N_{1}}} \int_{E_{N_{2}}} \int_{\epsilon_{4} \leq\left|x_{3}\right| \leq N_{4}} \frac{1}{|x|\left|x_{2}\right|\left|x_{3}\right|\left(\left|\frac{x_{1} x_{2} \xi}{x_{3}}\right|+\left|\frac{x_{3}}{x_{1} x_{2} \xi}\right|\right)^{\theta_{2}}} \mathrm{~d} x_{3} \mathrm{~d} x_{2} \mathrm{~d} x
\end{aligned}
$$




$$
\begin{aligned}
& +\int_{E_{N_{1}}} \int_{8 \leq\left|x_{2}\right| \leq N_{2}} \int_{\epsilon_{4} \leq\left|x_{3}\right| \leq N_{4}} \frac{1}{\left|x_{1}\right|\left|x_{2}\right|^{\theta_{1}+1}\left|x_{3}\right|\left(\left|\frac{x_{1} x_{2} \xi}{x_{3}}\right|+\left|\frac{x_{3}}{x_{1} x_{2} \xi}\right|\right)^{\theta_{2}}} \mathrm{~d} x_{3} \mathrm{~d} x_{2} \mathrm{~d} x_{1} \\
& \lesssim 1
\end{aligned}
$$

where we use the condition (RR) above on $\mathcal{K}$ with $\alpha=\beta=\gamma=0$ and $\alpha=0, \beta=$ $1, \gamma=0$, respectively.

To handle term $I_{1,2}$, we write

$$
\begin{aligned}
I_{1,2} \leq & \int_{E_{N_{1}}}\left|\int_{\epsilon_{2} \leq\left|x_{2}\right| \leq 8} \int_{8 \leq\left|x_{3}\right| \leq N_{4}} \frac{1}{\xi} \mathcal{K}\left(x_{1}, x_{2}, \frac{x_{3}}{\xi}\right) e^{-i x_{2}} e^{-i x_{3}} \mathrm{~d} x_{3} \mathrm{~d} x_{2}\right| \mathrm{d} x_{1} \\
& +\int_{E_{N_{1}}}\left|\int_{\epsilon_{2} \leq\left|x_{2}\right| \leq 8} \int_{\epsilon_{4} \leq\left|x_{3}\right| \leq 8} \frac{1}{\xi} \mathcal{K}\left(x_{1}, x_{2}, \frac{x_{3}}{\xi}\right) e^{-i x_{2}} e^{-i x_{3}} \mathrm{~d} x_{3} \mathrm{~d} x_{2}\right| \mathrm{d} x_{1} \\
& :=I_{1,2,1}+I_{1,2,2} .
\end{aligned}
$$

By Lemma 2.1 with $f\left(x_{3}\right)=\int_{\epsilon_{2} \leq\left|x_{2}\right| \leq 8} \frac{1}{\xi} \mathcal{K}\left(x_{1}, x_{2}, \frac{x_{3}}{\xi}\right) e^{-i x_{2}} \mathrm{~d} x_{2}$, we get

$$
\begin{aligned}
\left|I_{1,2,1}\right| \lesssim & \int_{E_{N_{1}}} \int_{E_{N_{2}}}\left|\int_{\epsilon_{2} \leq\left|x_{2}\right| \leq 8} \frac{1}{\xi} \mathcal{K}\left(x_{1}, x_{2}, \frac{x_{3}}{\xi}\right) e^{-i x_{2}} \mathrm{~d} x_{2}\right| \mathrm{d} x_{3} \mathrm{~d} x_{1} \\
& +\int_{E_{N_{1}}} \int_{8 \leq\left|x_{3}\right| \leq N_{4}} \mid \int_{\epsilon_{2} \leq\left|x_{2}\right| \leq 8} \frac{\Delta_{x_{3}, \pi}\left(\frac{1}{\xi} \mathcal{K}\left(x_{1}, x_{2}, \frac{x_{3}}{\xi}\right)\right) e^{-i x_{2}} \mathrm{~d} x_{2} \mid \mathrm{d} x_{3} \mathrm{~d} x_{1}}{\lesssim} \int_{E_{N_{1}}} \int_{E_{N_{2}}} \int_{\epsilon_{2} \leq\left|x_{2}\right| \leq 8} \frac{1}{\left|x_{1}\right|\left|x_{2}\right|\left|x_{3}\right|\left(\left|\frac{x_{1} x_{2} \xi}{x_{3}}\right|+\left|\frac{x_{3}}{x_{1} x_{2} \xi}\right|\right)^{\theta_{2}}} \mathrm{~d} x_{2} \mathrm{~d} x_{3} \mathrm{~d} x_{1} \\
& +\int_{E_{N_{1}}} \int_{8 \leq\left|x_{3}\right| \leq N_{4}} \int_{\epsilon_{2} \leq\left|x_{2}\right| \leq 8} \frac{1}{\left|x_{1}\right|\left|x_{2}\right|\left|x_{3}\right|^{1+\theta_{1}\left(\left|\frac{x_{1} x_{2} \xi}{x_{3}}\right|+\left|\frac{x_{3}}{x_{1} x_{2} \xi}\right|\right)^{\theta_{2}}} \mathrm{~d} x_{2} \mathrm{~d} x_{3} \mathrm{~d} x_{1}} \\
& \lesssim 1
\end{aligned}
$$

where we use the regularity condition (RR) above with $\alpha=\beta=\gamma=0$ and $\alpha=\beta=$ $0, \gamma=1$, respectively.

To estimate $I_{1,2,2}$, we note that

$$
\begin{aligned}
I_{1,2,2} \leq & \int_{E_{N_{1}}}\left|\int_{\epsilon_{2} \leq\left|x_{2}\right| \leq 8} \int_{\epsilon_{4} \leq\left|x_{3}\right| \leq 8} \frac{1}{\xi} \mathcal{K}\left(x_{1}, x_{2}, \frac{x_{3}}{\xi}\right)\left(e^{-i x_{2}} e^{-i x_{3}}-1\right) \mathrm{d} x_{3} \mathrm{~d} x_{2}\right| \mathrm{d} x_{1} \\
& +\int_{E_{N_{1}}}\left|\int_{\epsilon_{2} \leq\left|x_{2}\right| \leq 8} \int_{\epsilon_{4} \leq\left|x_{3}\right| \leq 8} \frac{1}{\xi} \mathcal{K}\left(x_{1}, x_{2}, \frac{x_{3}}{\xi}\right) \mathrm{d} x_{3} \mathrm{~d} x_{2}\right| \mathrm{d} x_{1} \\
& \lesssim \int_{E_{N_{1}}} \int_{\epsilon_{2} \leq\left|x_{2}\right| \leq 8} \int_{\epsilon_{4} \leq\left|x_{3}\right| \leq 8} \frac{\left|x_{2}\right|+\left|x_{3}\right|}{\left|x_{1}\right|\left|x_{2}\right|\left|x_{3}\right|\left(\left|\frac{x_{1} x_{2} \xi}{x_{3}}\right|+\left|\frac{x_{3}}{x_{1} x_{2} \xi}\right|\right)^{\theta_{2}}} \mathrm{~d} x_{3} \mathrm{~d} x_{2} \mathrm{~d} x_{1} \\
& +\int_{E_{N_{1}}} \frac{1}{\left|x_{1}\right|} \mathrm{d} x_{1} \lesssim 1,
\end{aligned}
$$


where we use the condition (RR) with $\alpha=\beta=\gamma=0$, and the cancellation condition (C1.c) with $\alpha=0$.

Next we consider $I_{2}$. Set $I_{2,1}$ and $I_{2,2}$ by

$$
\begin{aligned}
I_{2,1}= & \int_{8 \leq\left|x_{1}\right| \leq N_{1}} \mid \int_{8 \leq\left|x_{2}\right| \leq N_{2}} \int_{\epsilon_{4} \leq\left|x_{3}\right| \leq N_{4}} \Delta_{x_{1}, \pi} \\
& \times\left(\frac{1}{\xi} \mathcal{K}\left(x_{1}, x_{2}, \frac{x_{3}}{\xi}\right)\right) e^{-i x_{2}} e^{-i x_{3}} \mathrm{~d} x_{3} \mathrm{~d} x_{2} \mid \mathrm{d} x_{1}
\end{aligned}
$$

and

$$
\begin{aligned}
I_{2,2}= & \int_{8 \leq\left|x_{1}\right| \leq N_{1}} \mid \int_{\epsilon_{2} \leq\left|x_{2}\right| \leq 8} \int_{\epsilon_{4} \leq\left|x_{3}\right| \leq N_{4}} \Delta_{x_{1}, \pi} \\
& \times\left(\frac{1}{\xi} \mathcal{K}\left(x_{1}, x_{2}, \frac{x_{3}}{\xi}\right)\right) e^{-i x_{2}} e^{-i x_{3}} \mathrm{~d} x_{3} \mathrm{~d} x_{2} \mid \mathrm{d} x_{1} .
\end{aligned}
$$

Then $I_{2} \leq\left|I_{2,1}\right|+\left|I_{2,2}\right|$. Similarly, applying Lemma 2.1 with

$$
f\left(x_{2}\right)=\int_{\epsilon_{4} \leq\left|x_{3}\right| \leq N_{4}} \Delta_{x_{1}, \pi}\left(\frac{1}{\xi} \mathcal{K}\left(x_{1}, x_{2}, \frac{x_{3}}{\xi}\right)\right) e^{-i x_{3}} \mathrm{~d} x_{3},
$$

we obtain

$$
\begin{aligned}
\left|I_{2,1}\right| \lesssim & \int_{8 \leq\left|x_{1}\right| \leq N_{1}} \int_{E_{N_{2}}}\left|\int_{\epsilon_{4} \leq\left|x_{3}\right| \leq N_{4}} \Delta_{x_{1}, \pi}\left(\frac{1}{\xi} \mathcal{K}\left(x_{1}, x_{2}, \frac{x_{3}}{\xi}\right)\right) e^{-i x_{3}} \mathrm{~d} x_{3}\right| \mathrm{d} x_{2} \mathrm{~d} x_{1} \\
& +\int_{8 \leq\left|x_{1}\right| \leq N_{1}} \int_{8 \leq\left|x_{2}\right| \leq N_{2}} \mid \int_{\epsilon_{4} \leq\left|x_{3}\right| \leq N_{4}} \Delta_{x_{2}, \pi} \Delta_{x_{1}, \pi}\left(\frac{1}{\xi} \mathcal{K}\left(x_{1}, x_{2}, \frac{x_{3}}{\xi}\right)\right) \\
& \times e^{-i x_{3}} \mathrm{~d} x_{3} \mid \mathrm{d} x_{2} \mathrm{~d} x_{1} \\
\leq & \int_{8 \leq\left|x_{1}\right| \leq N_{1}} \int_{E_{N_{2}}} \int_{\epsilon_{4} \leq\left|x_{3}\right| \leq N_{4}} \frac{1}{\left|x_{1}\right|^{1+\theta_{1}}\left|x_{2}\right|\left|x_{3}\right|\left(\left|\frac{x_{1} x_{2} \xi}{x_{3}}\right|+\left|\frac{x_{3}}{x_{1} x_{2} \xi}\right|\right)^{\theta_{2}}} \mathrm{~d} x_{3} \mathrm{~d} x_{2} \mathrm{~d} x_{1} \\
& +\int_{8 \leq\left|x_{1}\right| \leq N_{1}} \int_{8 \leq\left|x_{2}\right| \leq N_{2}} \int_{\epsilon_{4} \leq\left|x_{3}\right| \leq N_{4}} \frac{1}{\left|x_{1}\right|^{1+\theta_{1}}\left|x_{2}\right|^{1+\theta_{1}}\left|x_{3}\right|\left(\left|\frac{x_{1} x_{2} \xi}{x_{3}}\right|+\left|\frac{x_{3}}{x_{1} x_{2} \xi}\right|\right)^{\theta_{2}}} \\
& \times \mathrm{d} x_{3} \mathrm{~d} x_{2} \mathrm{~d} x_{1} \lesssim 1,
\end{aligned}
$$

where we use the condition (RR) above with $\alpha=1, \beta=\gamma=0$ and $\alpha=\beta=1, \gamma=$ 0 , respectively.

For term $I_{2,2}$, note that

$$
\begin{aligned}
I_{2,2} \leq & \int_{8 \leq\left|x_{1}\right| \leq N_{1}} \mid \int_{\epsilon_{2} \leq\left|x_{2}\right| \leq 8} \int_{8 \leq\left|x_{3}\right| \leq N_{4}} \Delta_{x_{1}, \pi}\left(\frac{1}{\xi} \mathcal{K}\left(x_{1}, x_{2}, \frac{x_{3}}{\xi}\right)\right) \\
& \times e^{-i x_{2}} e^{-i x_{3}} \mathrm{~d} x_{3} \mathrm{~d} x_{2} \mid \mathrm{d} x_{1}
\end{aligned}
$$




$$
\begin{aligned}
& +\int_{8 \leq\left|x_{1}\right| \leq N_{1}} \mid \int_{\epsilon_{2} \leq\left|x_{2}\right| \leq 8} \int_{\epsilon_{4} \leq\left|x_{3}\right| \leq 8} \Delta_{x_{1}, \pi}\left(\frac{1}{\xi} \mathcal{K}\left(x_{1}, x_{2}, \frac{x_{3}}{\xi}\right)\right) \\
& \times e^{-i x_{2}} e^{-i x_{3}} \mathrm{~d} x_{3} \mathrm{~d} x_{2} \mid \mathrm{d} x_{1} \\
& :=I_{2,2,1}+I_{2,2,2} .
\end{aligned}
$$

By Lemma 2.1 with $f\left(x_{3}\right)=\int_{\epsilon_{2} \leq\left|x_{2}\right| \leq 8} \Delta_{x_{1}, \pi}\left(\frac{1}{\xi} \mathcal{K}\left(x_{1}, x_{2}, \frac{x_{3}}{\xi}\right)\right) e^{-i x_{2}} \mathrm{~d} x_{2}$, we have

$$
\begin{aligned}
\left|I_{2,2,1}\right| \lesssim & \int_{8 \leq\left|x_{1}\right| \leq N_{1}} \int_{E_{N_{4}}}\left|\int_{\epsilon_{2} \leq\left|x_{2}\right| \leq 8} \Delta_{x_{1}, \pi}\left(\frac{1}{\xi} \mathcal{K}\left(x_{1}, x_{2}, \frac{x_{3}}{\xi}\right)\right) e^{-i x_{2}} \mathrm{~d} x_{2}\right| \mathrm{d} x_{3} \mathrm{~d} x_{1} \\
& +\int_{8 \leq\left|x_{1}\right| \leq N_{1}} \int_{8 \leq\left|x_{3}\right| \leq N_{4}} \mid \int_{\epsilon_{2} \leq\left|x_{2}\right| \leq 8} \Delta_{x_{3}, \pi} \Delta_{x_{1}, \pi}\left(\frac{1}{\xi} \mathcal{K}\left(x_{1}, x_{2}, \frac{x_{3}}{\xi}\right)\right) \\
& \times e^{-i x_{2}} \mathrm{~d} x_{2} \mid \mathrm{d} x_{3} \mathrm{~d} x_{1} \\
\leq & \int_{8 \leq\left|x_{1}\right| \leq N_{1}} \int_{E_{N_{4}}} \int_{\epsilon_{2} \leq\left|x_{2}\right| \leq 8} \frac{1}{\left|x_{1}\right|^{1+\theta_{1}}\left|x_{2}\right|\left|x_{3}\right|\left(\left|\frac{x_{1} x_{2} \xi}{x_{3}}\right|+\left|\frac{x_{3}}{x_{1} x_{2} \xi}\right|\right)^{\theta_{2}}} \mathrm{~d} x_{2} \mathrm{~d} x_{3} \mathrm{~d} x_{1} \\
& +\int_{8 \leq\left|x_{1}\right| \leq N_{1}} \int_{8 \leq\left|x_{3}\right| \leq N_{4}} \int_{\epsilon_{2} \leq\left|x_{2}\right| \leq 8} \frac{1}{\left|x_{1}\right|^{1+\theta_{1}}\left|x_{2}\right|\left|x_{3}\right|+\theta_{1}\left(\left|\frac{x_{1} x_{2} \xi}{x_{3}}\right|+\left|\frac{x_{3}}{x_{1} x_{2} \xi}\right|\right)^{\theta_{2}}} \\
& \times \mathrm{d} x_{2} \mathrm{~d} x_{3} \mathrm{~d} x_{1} \lesssim 1,
\end{aligned}
$$

where we use the conditions (RR) above with $\alpha=1, \beta=\gamma=0$ and $\alpha=\gamma=1, \beta=$ 0 , respectively.

The estimate for term $I_{2,2,2}$ follows from a similar way as term $I_{1,2,2}$. Indeed,

$$
\begin{aligned}
& I_{2,2,2}=\int_{8 \leq\left|x_{1}\right| \leq N_{1}}\left|\int_{\epsilon_{2} \leq\left|x_{2}\right| \leq 8} \int_{\epsilon_{4} \leq\left|x_{3}\right| \leq 8} \Delta_{x_{1}, \pi}\left(\frac{1}{\xi} \mathcal{K}\left(x_{1}, x_{2}, \frac{x_{3}}{\xi}\right)\right)\left(e^{-i x_{2}} e^{-i x_{3}}-1\right) \mathrm{d} x_{3} \mathrm{~d} x_{2}\right| \mathrm{d} x_{1} \\
& +\int_{8 \leq\left|x_{1}\right| \leq N_{1}} \mid \int_{\epsilon_{2} \leq\left|x_{2}\right| \leq 8} \int_{\epsilon_{4} \leq\left|x_{3}\right| \leq 8} \Delta_{x_{1}, \pi}\left(\frac{1}{\xi} \mathcal{K}\left(x_{1}, x_{2}, \frac{x_{3}}{\xi}\right)\right) \\
& \times \mathrm{d} x_{3} \mathrm{~d} x_{2} \mid \mathrm{d} x_{1} \\
& \lesssim \int_{8 \leq\left|x_{1}\right| \leq N_{1}} \int_{\epsilon_{2} \leq\left|x_{2}\right| \leq 8} \int_{\epsilon_{4} \leq\left|x_{3}\right| \leq 8} \frac{\left|x_{2}\right|+\left|x_{3}\right|}{\left|x_{1}\right|^{1+\theta_{1}}\left|x_{2}\right|\left|x_{3}\right|\left(\left|\frac{x_{1} x_{2} \xi}{x_{3}}\right|+\left|\frac{x_{3}}{x_{1} x_{2} \xi}\right|\right)^{\theta_{2}}} \mathrm{~d} x_{2} \mathrm{~d} x_{3} \mathrm{~d} x_{1} \\
& +\int_{8 \leq\left|x_{1}\right| \leq N_{1}} \frac{1}{\left|x_{1}\right|^{1+\theta_{1}}} \mathrm{~d} x_{1} \lesssim 1 \text {, }
\end{aligned}
$$

where we use the condition (RR) with $\alpha=1, \beta=\gamma=0$ and the condition (C1.c), respectively.

Now we turn to the estimate for term $I I$. We first write

$$
\begin{aligned}
I I= & \int_{\epsilon_{4} \leq\left|x_{3}\right| \leq N_{4}} \int_{\epsilon_{2} \leq\left|x_{2}\right| \leq N_{2}} \int_{\epsilon_{1} \leq\left|x_{1}\right| \leq 8} \frac{1}{\xi} \mathcal{K}\left(x_{1}, x_{2}, \frac{x_{3}}{\xi}\right)\left(e^{-i x_{1}}-1\right) e^{-i x_{2}} \\
& \times e^{-i x_{3}} \mathrm{~d} x_{1} \mathrm{~d} x_{2} \mathrm{~d} x_{3}
\end{aligned}
$$




$$
\begin{aligned}
& +\int_{\epsilon_{4} \leq\left|x_{3}\right| \leq N_{4}} \int_{\epsilon_{2} \leq\left|x_{2}\right| \leq N_{2}} \int_{\epsilon_{1} \leq\left|x_{1}\right| \leq 8} \frac{1}{\xi} \mathcal{K}\left(x_{1}, x_{2}, \frac{x_{3}}{\xi}\right) \\
& \times e^{-i x_{2}} e^{-i x_{3}} \mathrm{~d} x_{1} \mathrm{~d} x_{2} \mathrm{~d} x_{3} \\
& :=I I_{1}+I I_{2} .
\end{aligned}
$$

We further write

$$
\begin{aligned}
\left|I I_{1}\right|= & \int_{\epsilon_{4} \leq\left|x_{3}\right| \leq N_{4}} \int_{8 \leq\left|x_{2}\right| \leq N_{2}} \int_{\epsilon_{1} \leq\left|x_{1}\right| \leq 8} \frac{1}{\xi} \mathcal{K}\left(x_{1}, x_{2}, \frac{x_{3}}{\xi}\right)\left(e^{-i x_{1}}-1\right) e^{-i x_{2}} e^{-i x_{3}} \mathrm{~d} x_{1} \mathrm{~d} x_{2} \mathrm{~d} x_{3} \\
& +\int_{\epsilon_{4} \leq\left|x_{3}\right| \leq N_{4}} \int_{\epsilon_{2} \leq\left|x_{2}\right| \leq 8} \int_{\epsilon_{1} \leq\left|x_{1}\right| \leq 8} \frac{1}{\xi} \mathcal{K}\left(x_{1}, x_{2}, \frac{x_{3}}{\xi}\right)\left(e^{-i x_{1}}-1\right) e^{-i x_{2}} e^{-i x_{3}} \mathrm{~d} x_{1} \mathrm{~d} x_{2} \mathrm{~d} x_{3} \\
& :=I I_{1,1}+I I_{1,2} .
\end{aligned}
$$

For term $I I_{1,1}$, using Lemma 2.1 with $f\left(x_{2}\right)=\int_{\epsilon_{4} \leq\left|x_{3}\right| \leq N_{4}} \int_{\epsilon_{1} \leq\left|x_{1}\right| \leq 8} \frac{1}{\xi} \mathcal{K}\left(x_{1}, x_{2}, \frac{x_{3}}{\xi}\right)$ $\left(e^{-i x_{1}}-1\right) e^{-i x_{3}} \mathrm{~d} x_{1} \mathrm{~d} x_{3}$, we obtain

$$
\begin{aligned}
\left|I I_{1,1}\right| \lesssim & \int_{E_{N_{2}}}\left|\int_{\epsilon_{4} \leq\left|x_{3}\right| \leq N_{4}} \int_{\epsilon_{1} \leq\left|x_{1}\right| \leq 8} \frac{1}{\xi} \mathcal{K}\left(x_{1}, x_{2}, \frac{x_{3}}{\xi}\right)\left(e^{-i x_{1}}-1\right) e^{-i x_{3}} \mathrm{~d} x_{1} \mathrm{~d} x_{3}\right| \mathrm{d} x_{2} \\
& +\int_{8 \leq\left|x_{2}\right| \leq N_{2}} \mid \int_{\epsilon_{4} \leq\left|x_{3}\right| \leq N_{4}} \int_{\epsilon_{1} \leq\left|x_{1}\right| \leq 8} \Delta_{x_{2}, \pi}\left(\frac{1}{\xi} \mathcal{K}\left(x_{1}, x_{2}, \frac{x_{3}}{\xi}\right)\right) \\
& \times\left(e^{-i x_{1}}-1\right) e^{-i x_{3}} \mathrm{~d} x_{1} \mathrm{~d} x_{3} \mid \mathrm{d} x_{2} \\
\lesssim & \int_{E_{N_{2}}} \int_{\epsilon_{4} \leq\left|x_{3}\right| \leq N_{4}} \int_{\epsilon_{1} \leq\left|x_{1}\right| \leq 8} \frac{1}{\left|x_{2}\right|\left|x_{3}\right|\left(\left|\frac{x_{1} x_{2} \xi}{x_{3}}\right|+\left|\frac{x_{3}}{x_{1} x_{2} \xi}\right|\right)^{\theta_{2}}} \mathrm{~d} x_{1} \mathrm{~d} x_{3} \mathrm{~d} x_{2} \\
& +\int_{8 \leq\left|x_{2}\right| \leq N_{2}} \int_{\epsilon_{4} \leq\left|x_{3}\right| \leq N_{4}} \int_{\epsilon_{1} \leq\left|x_{1}\right| \leq 8} \frac{1}{\left|x_{2}\right|^{1+\theta_{1}}\left|x_{3}\right|\left(\left|\frac{x_{1} x_{2} \xi}{x_{3}}\right|+\left|\frac{x_{3}}{x_{1} x_{2} \xi}\right|\right)^{\theta_{2}}} \mathrm{~d} x_{1} \mathrm{~d} x_{3} \mathrm{~d} x_{2},
\end{aligned}
$$

where we use the condition (RR) above for $\alpha=\beta=\gamma=0$ and $\beta=1, \alpha=\gamma=0$, respectively.

Similarly,

$$
\begin{aligned}
I I_{1,2}= & \int_{8 \leq\left|x_{3}\right| \leq N_{4}} \int_{\epsilon_{2} \leq\left|x_{2}\right| \leq 8} \int_{\epsilon_{1} \leq\left|x_{1}\right| \leq 8} \frac{1}{\xi} \mathcal{K}\left(x_{1}, x_{2}, \frac{x_{3}}{\xi}\right) \\
& \times\left(e^{-i x_{1}}-1\right) e^{-i x_{2}} e^{-i x_{3}} \mathrm{~d} x_{1} \mathrm{~d} x_{2} \mathrm{~d} x_{3} \\
& +\int_{\epsilon_{4} \leq\left|x_{3}\right| \leq 8} \int_{\epsilon_{2} \leq\left|x_{2}\right| \leq 8} \int_{\epsilon_{1} \leq\left|x_{1}\right| \leq 8} \frac{1}{\xi} \mathcal{K}\left(x_{1}, x_{2}, \frac{x_{3}}{\xi}\right) \\
& \times\left(e^{-i x_{1}}-1\right) e^{-i x_{2}} e^{-i x_{3}} \mathrm{~d} x_{1} \mathrm{~d} x_{2} \mathrm{~d} x_{3}:=I I_{1,2,1}+I I_{1,2,2}
\end{aligned}
$$

The bounds of $I I_{1,2,1}$ and $I I_{1,2,2}$ then follow from the similar estimates as terms $I_{2,2,1}$ and $I_{2,2,2}$, respectively. 
Finally, we estimate term $I I_{2}$. Denote $I I_{2}=I I_{2,1}+I I_{2,2}$, where

$$
I I_{2,1}=\int_{\epsilon_{4} \leq\left|x_{3}\right| \leq N_{4}} \int_{8 \leq\left|x_{2}\right| \leq N_{2}} \int_{\epsilon_{1} \leq\left|x_{1}\right| \leq 8} \frac{1}{\xi} \mathcal{K}\left(x_{1}, x_{2}, \frac{x_{3}}{\xi}\right) e^{-i x_{2}} e^{-i x_{3}} \mathrm{~d} x_{1} \mathrm{~d} x_{2} \mathrm{~d} x_{3}
$$

and

$$
I I_{2,2}=\int_{\epsilon_{4} \leq\left|x_{3}\right| \leq N_{4}} \int_{\epsilon_{2} \leq\left|x_{2}\right| \leq 8} \int_{\epsilon_{1} \leq\left|x_{1}\right| \leq 8} \frac{1}{\xi} \mathcal{K}\left(x_{1}, x_{2}, \frac{x_{3}}{\xi}\right) e^{-i x_{2}} e^{-i x_{3}} \mathrm{~d} x_{1} \mathrm{~d} x_{2} \mathrm{~d} x_{3}
$$

Note that

$$
\begin{aligned}
\left|I I_{2,1}\right|= & \int_{8 \leq\left|x_{3}\right| \leq N_{4}} \int_{8 \leq\left|x_{2}\right| \leq N_{2}} \int_{\epsilon_{1} \leq\left|x_{1}\right| \leq 8} \frac{1}{\xi} \mathcal{K}\left(x_{1}, x_{2}, \frac{x_{3}}{\xi}\right) e^{-i x_{2}} e^{-i x_{3}} \mathrm{~d} x_{1} \mathrm{~d} x_{2} \mathrm{~d} x_{3} \\
& +\int_{\epsilon_{4} \leq\left|x_{3}\right| \leq 8} \int_{8 \leq\left|x_{2}\right| \leq N_{2}} \int_{\epsilon_{1} \leq\left|x_{1}\right| \leq 8} \frac{1}{\xi} \mathcal{K}\left(x_{1}, x_{2}, \frac{x_{3}}{\xi}\right) e^{-i x_{2}} e^{-i x_{3}} \mathrm{~d} x_{1} \mathrm{~d} x_{2} \mathrm{~d} x_{3} . \\
:= & I I_{2,1,1}+I I_{2,1,2}
\end{aligned}
$$

Applying Lemma 2.1 with $f\left(x_{2}\right)=\int_{8 \leq\left|x_{3}\right| \leq N_{4}} \int_{\epsilon_{1} \leq\left|x_{1}\right| \leq 8} \frac{1}{\xi} \mathcal{K}\left(x_{1}, x_{2}, \frac{x_{3}}{\xi}\right) e^{-i x_{3}} \mathrm{~d} x_{1} \mathrm{~d} x_{3}$ first, and then $f\left(x_{3}\right)=\int_{\epsilon_{1} \leq\left|x_{1}\right| \leq 8} \frac{1}{\xi} \mathcal{K}\left(x_{1}, x_{2}, \frac{x_{3}}{\xi}\right) \mathrm{d} x_{1}$, combining with the condition (RR), we obtain

$$
\begin{aligned}
\left|I I_{2,1,1}\right| \lesssim & \int_{E_{N_{2}}}\left|\int_{8 \leq\left|x_{3}\right| \leq N_{4}} \int_{\epsilon_{1} \leq\left|x_{1}\right| \leq 8} \frac{1}{\xi} \mathcal{K}\left(x_{1}, x_{2}, \frac{x_{3}}{\xi}\right) e^{-i x_{3}} \mathrm{~d} x_{1} \mathrm{~d} x_{3}\right| \mathrm{d} x_{2} \\
& +\int_{8 \leq\left|x_{2}\right| \leq N_{2}} \mid \int_{8 \leq\left|x_{3}\right| \leq N_{4}} \int_{\epsilon_{1} \leq\left|x_{1}\right| \leq 8} \Delta_{x_{2}, \pi}\left(\frac{1}{\xi} \mathcal{K}\left(x_{1}, x_{2}, \frac{x_{3}}{\xi}\right)\right) \\
& \times e^{-i x_{3}} \mathrm{~d} x_{1} \mathrm{~d} x_{3} \mid \mathrm{d} x_{2} \\
& \lesssim \int_{E_{N_{2}}} \int_{E_{N_{4}}}\left|\int_{\epsilon_{1} \leq\left|x_{1}\right| \leq 8} \frac{1}{\xi} \mathcal{K}\left(x_{1}, x_{2}, \frac{x_{3}}{\xi}\right) e^{-i x_{3}} \mathrm{~d} x_{1}\right| \mathrm{d} x_{3} \mathrm{~d} x_{2} \\
& +\int_{E_{N_{2}}} \int_{8 \leq\left|x_{3}\right| \leq N_{4}}\left|\int_{\epsilon_{1} \leq\left|x_{1}\right| \leq 8} \Delta_{x_{3}, \pi}\left(\frac{1}{\xi} \mathcal{K}\left(x_{1}, x_{2}, \frac{x_{3}}{\xi}\right)\right) e^{-i x_{3}} \mathrm{~d} x_{1}\right| \mathrm{d} x_{3} \mathrm{~d} x_{2} \\
& +\int_{8 \leq\left|x_{2}\right| \leq N_{2}} \int_{E_{N_{4}}}\left|\int_{\epsilon_{1} \leq\left|x_{1}\right| \leq 8} \Delta_{x_{2}, \pi}\left(\frac{1}{\xi} \mathcal{K}\left(x_{1}, x_{2}, \frac{x_{3}}{\xi}\right)\right) \mathrm{d} x_{1}\right| \mathrm{d} x_{3} \mathrm{~d} x_{2} \\
& +\int_{8 \leq\left|x_{2}\right| \leq N_{2}} \int_{8 \leq\left|x_{3}\right| \leq N_{4}} \mid \int_{\epsilon_{1} \leq\left|x_{1}\right| \leq 8} \Delta_{x_{3}, \pi} \Delta_{x_{2}, \pi}\left(\frac{1}{\xi} \mathcal{K}\left(x_{1}, x_{2}, \frac{x_{3}}{\xi}\right)\right) \\
& \times \mathrm{d} x_{1} \mid \mathrm{d} x_{3} \mathrm{~d} x_{2} \lesssim 1 .
\end{aligned}
$$


To estimate $I I_{2,1,2}$, inserting $e^{-i x_{3}}=\left[e^{-i x_{3}}-1\right]+1$ and then applying Lemma 2.1, we get

$$
\begin{aligned}
I I_{2,1,2} \lesssim & \mid \int_{8 \leq\left|x_{2}\right| \leq N_{2}} \int_{\epsilon_{1} \leq\left|x_{1}\right| \leq 8} \int_{\epsilon_{4} \leq\left|x_{3}\right| \leq 8} \frac{1}{\xi} \mathcal{K}\left(x_{1}, x_{2}, \frac{x_{3}}{\xi}\right) e^{-i x_{2}}\left(e^{-i x_{3}}-1\right) \\
& \mathrm{d} x_{3} \mathrm{~d} x_{1} \mathrm{~d} x_{2} \mid \\
& +\left|\int_{8 \leq\left|x_{2}\right| \leq N_{2}} \int_{\epsilon_{1} \leq\left|x_{1}\right| \leq 8} \int_{\epsilon_{4} \leq\left|x_{3}\right| \leq 8} \frac{1}{\xi} \mathcal{K}\left(x_{1}, x_{2}, \frac{x_{3}}{\xi}\right) e^{-i x_{2}} \mathrm{~d} x_{3} \mathrm{~d} x_{1} \mathrm{~d} x_{2}\right| \\
\lesssim & \int_{E_{N_{2}}} \int_{\epsilon_{1} \leq\left|x_{1}\right| \leq 8} \int_{\epsilon_{4} \leq\left|x_{3}\right| \leq 8}\left|\frac{1}{\xi} \mathcal{K}\left(x_{1}, x_{2}, \frac{x_{3}}{\xi}\right)\right|\left|x_{3}\right| \mathrm{d} x_{3} \mathrm{~d} x_{1} \mathrm{~d} x_{2} \\
& +\int_{8 \leq\left|x_{2}\right| \leq N_{2}} \int_{\epsilon_{1} \leq\left|x_{1}\right| \leq 8} \int_{\epsilon_{4} \leq\left|x_{3}\right| \leq 8}\left|\Delta_{x_{2}, \pi}\left(\frac{1}{\xi} \mathcal{K}\left(x_{1}, x_{2}, \frac{x_{3}}{\xi}\right)\right)\right|\left|x_{3}\right| \mathrm{d} x_{3} \mathrm{~d} x_{1} \mathrm{~d} x_{2} \\
& +\int_{E_{N_{2}}}\left|\int_{\epsilon_{1} \leq\left|x_{1}\right| \leq 8} \int_{\epsilon_{4} \leq\left|x_{3}\right| \leq 8} \frac{1}{\xi} \mathcal{K}\left(x_{1}, x_{2}, \frac{x_{3}}{\xi}\right) \mathrm{d} x_{3} \mathrm{~d} x_{1}\right| \mathrm{d} x_{2} \\
& +\int_{8 \leq\left|x_{2}\right| \leq N_{2}}\left|\int_{\epsilon_{1} \leq\left|x_{1}\right| \leq 8} \int_{\epsilon_{4} \leq\left|x_{3}\right| \leq 8} \Delta_{x_{2}, \pi}\left(\frac{1}{\xi} \mathcal{K}\left(x_{1}, x_{2}, \frac{x_{3}}{\xi}\right)\right) \mathrm{d} x_{3} \mathrm{~d} x_{1}\right| \mathrm{d} x_{2} .
\end{aligned}
$$

The required bound then follows from the conditions (RR) for the first two integrals while the condition (C1.d) for the last two integrals.

For $I I_{2,2}$, splitting the set $\left\{\epsilon_{4} \leq\left|x_{3}\right| \leq N_{4}\right\}$ into two parts $\left\{\epsilon_{4} \leq\left|x_{3}\right| \leq 8\right\}$ and $\{8 \leq$ $\left.\left|x_{3}\right| \leq N_{4}\right\}$, and inserting $e^{-i x_{2}} e^{-i x_{3}}=\left(e^{-i x_{2}}-1\right)\left(e^{-i x_{3}}-1\right)+\left(e^{-i x_{2}}-1\right)+\left(e^{-i x_{3}}-\right.$ $1)+1$ for the integral over the first set and $e^{-i x_{2}} e^{-i x_{3}}=\left(e^{-i x_{2}}-1\right) e^{-i x_{3}}+e^{-i x_{3}}$ for the integral over the second set, we obtain

$$
\begin{aligned}
I I_{2,2} \lesssim & \left|\int_{\epsilon_{4} \leq\left|x_{3}\right| \leq 8} \int_{\epsilon_{2} \leq\left|x_{2}\right| \leq 8} \int_{\epsilon_{1} \leq\left|x_{1}\right| \leq 8} \frac{1}{\xi} \mathcal{K}\left(x_{1}, x_{2}, \frac{x_{3}}{\xi}\right)\left(e^{-i x_{2}}-1\right)\left(e^{-i x_{3}}-1\right) \mathrm{d} x_{1} \mathrm{~d} x_{2} \mathrm{~d} x_{3}\right| \\
& +\left|\int_{\epsilon_{4} \leq\left|x_{3}\right| \leq 8} \int_{\epsilon_{2} \leq\left|x_{2}\right| \leq 8} \int_{\epsilon_{1} \leq\left|x_{1}\right| \leq 8} \frac{1}{\xi} \mathcal{K}\left(x_{1}, x_{2}, \frac{x_{3}}{\xi}\right)\left(e^{-i x_{2}}-1\right) \mathrm{d} x_{1} \mathrm{~d} x_{2} \mathrm{~d} x_{3}\right| \\
& +\left|\int_{\epsilon_{4} \leq\left|x_{3}\right| \leq 8} \int_{\epsilon_{2} \leq\left|x_{2}\right| \leq 8} \int_{\epsilon_{1} \leq\left|x_{1}\right| \leq 8} \frac{1}{\xi} \mathcal{K}\left(x_{1}, x_{2}, \frac{x_{3}}{\xi}\right)\left(e^{-i x_{3}}-1\right) \mathrm{d} x_{1} \mathrm{~d} x_{2} \mathrm{~d} x_{3}\right| \\
& +\left|\int_{\epsilon_{4} \leq\left|x_{3}\right| \leq 8} \int_{\epsilon_{2} \leq\left|x_{2}\right| \leq 8} \int_{\epsilon_{1} \leq\left|x_{1}\right| \leq 8} \frac{1}{\xi} \mathcal{K}\left(x_{1}, x_{2}, \frac{x_{3}}{\xi}\right) \mathrm{d} x_{1} \mathrm{~d} x_{2} \mathrm{~d} x_{3}\right| \\
& +\left|\int_{8 \leq\left|x_{3}\right| \leq N_{4}} \int_{\epsilon_{2} \leq\left|x_{2}\right| \leq 8} \int_{\epsilon_{1} \leq\left|x_{1}\right| \leq 8} \frac{1}{\xi} \mathcal{K}\left(x_{1}, x_{2}, \frac{x_{3}}{\xi}\right)\left(e^{-i x_{2}}-1\right) e^{-i x_{3}} \mathrm{~d} x_{1} \mathrm{~d} x_{2} \mathrm{~d} x_{3}\right| \\
& +\left|\int_{8 \leq\left|x_{3}\right| \leq N_{4}} \int_{\epsilon_{2} \leq\left|x_{2}\right| \leq 8} \int_{\epsilon_{1} \leq\left|x_{1}\right| \leq 8} \frac{1}{\xi} \mathcal{K}\left(x_{1}, x_{2}, \frac{x_{3}}{\xi}\right) e^{-i x_{3}} \mathrm{~d} x_{1} \mathrm{~d} x_{2} \mathrm{~d} x_{3}\right| .
\end{aligned}
$$

The first four items follow from the conditions (RR), (C1.d), (C1.b), and (C1.a), respectively. To estimate the fifth and sixth terms, applying Lemma 2.1, we get 


$$
\begin{aligned}
& \left|\int_{8 \leq\left|x_{3}\right| \leq N_{4}} \int_{\epsilon_{2} \leq\left|x_{2}\right| \leq 8} \int_{\epsilon_{1} \leq\left|x_{1}\right| \leq 8} \frac{1}{\xi} \mathcal{K}\left(x_{1}, x_{2}, \frac{x_{3}}{\xi}\right)\left(e^{-i x_{2}}-1\right) e^{-i x_{3}} \mathrm{~d} x_{1} \mathrm{~d} x_{2} \mathrm{~d} x_{3}\right| \\
& \quad+\left|\int_{8 \leq\left|x_{3}\right| \leq N_{4}} \int_{\epsilon_{2} \leq\left|x_{2}\right| \leq 8} \int_{\epsilon_{1} \leq\left|x_{1}\right| \leq 8} \frac{1}{\xi} \mathcal{K}\left(x_{1}, x_{2}, \frac{x_{3}}{\xi}\right) e^{-i x_{3}} \mathrm{~d} x_{1} \mathrm{~d} x_{2} \mathrm{~d} x_{3}\right| \\
& \quad \lesssim \int_{E_{N_{4}}}\left|\int_{\epsilon_{2} \leq\left|x_{2}\right| \leq 8} \int_{\epsilon_{1} \leq\left|x_{1}\right| \leq 8} \frac{1}{\xi} \mathcal{K}\left(x_{1}, x_{2}, \frac{x_{3}}{\xi}\right)\left(e^{-i x_{2}}-1\right) \mathrm{d} x_{1} \mathrm{~d} x_{2}\right| \mathrm{d} x_{3} \\
& \quad+\int_{8 \leq\left|x_{3}\right| \leq N_{4}}\left|\int_{\epsilon_{2} \leq\left|x_{2}\right| \leq 8} \int_{\epsilon_{1} \leq\left|x_{1}\right| \leq 8} \Delta_{x_{3}, \pi}\left(\frac{1}{\xi} \mathcal{K}\left(x_{1}, x_{2}, \frac{x_{3}}{\xi}\right)\right)\left(e^{-i x_{2}}-1\right) \mathrm{d} x_{1} \mathrm{~d} x_{2}\right| \mathrm{d} x_{3} \\
& \quad+\int_{E_{N_{4}}}\left|\int_{\epsilon_{2} \leq\left|x_{2}\right| \leq 8} \int_{\epsilon_{1} \leq\left|x_{1}\right| \leq 8} \frac{1}{\xi} \mathcal{K}\left(x_{1}, x_{2}, \frac{x_{3}}{\xi}\right) \mathrm{d} x_{1} \mathrm{~d} x_{2}\right| \mathrm{d} x_{3} \\
& \quad+\int_{8 \leq\left|x_{3}\right| \leq N_{4}}\left|\int_{\epsilon_{2} \leq\left|x_{2}\right| \leq 8} \int_{\epsilon_{1} \leq\left|x_{1}\right| \leq 8} \Delta_{x_{3}, \pi}\left(\frac{1}{\xi} \mathcal{K}\left(x_{1}, x_{2}, \frac{x_{3}}{\xi}\right)\right) \mathrm{d} x_{1} \mathrm{~d} x_{2}\right| \mathrm{d} x_{3} \lesssim 1,
\end{aligned}
$$

where we use the condition (RR) for the first two terms and (C1.b) for the last two terms above. Thus, these estimates yield the bound of $I I_{2,2}$ and hence the required bound for term $I I$. The $L^{2}$ boundedness of $\mathcal{K}_{\epsilon}^{N} * f$ follows.

Proof of Corollary 1.2 It suffices to show that $\mathcal{K}_{\epsilon}^{N} * f$ converges in $L^{2}\left(\mathbb{R}^{3}\right)$, as $\epsilon_{1}, \epsilon_{2}, \epsilon_{3} \rightarrow 0$ and $N_{1}, N_{2}, N_{3} \rightarrow \infty$, for a dense subset of $L^{2}\left(\mathbb{R}^{3}\right)$. For this purpose, we consider smooth functions $f$ having compact support. We may assume that $\epsilon_{1}, \epsilon_{2}, \epsilon_{3}<1$ and $N_{1}, N_{2}, N_{3}>1$.

We write $\left.\iiint_{\mathbb{R}^{3}} \mathcal{K}_{\epsilon}^{N}(u) f(x-u)\right) d u$ as a sum of eight terms; that is, the integrals over the sets $(i)\left|u_{1}\right| \leq 1,\left|u_{2}\right| \leq 1,\left|u_{3}\right| \leq 1 ;($ ii $)\left|u_{1}\right| \leq 1,\left|u_{2}\right| \leq 1,\left|u_{3}\right|>1 ;($ iii $)\left|u_{1}\right| \leq$ $1,\left|u_{2}\right|>1,\left|u_{3}\right| \leq 1 ;(i v)\left|u_{1}\right| \leq 1,\left|u_{2}\right|>1,\left|u_{3}\right|>1 ;(v)\left|u_{1}\right|>1,\left|u_{2}\right| \leq 1,\left|u_{3}\right| \leq$ $1 ;(v i)\left|u_{1}\right|>1,\left|u_{2}\right| \leq 1,\left|u_{3}\right|>1 ;($ vii $)\left|u_{1}\right|>1,\left|u_{2}\right|>1,\left|u_{3}\right| \leq 1 ;($ viii $)\left|u_{1}\right|>$ $1,\left|u_{2}\right|>1,\left|u_{3}\right|>1$. Inserting

$$
\begin{aligned}
f(x-u)= & {\left[f\left(x_{1}-u_{1}, x_{2}-u_{2}, x_{3}-u_{3}\right)-f\left(x_{1}, x_{2}-u_{2}, x_{3}-u_{3}\right)\right.} \\
& -f\left(x_{1}-u_{1}, x_{2}, x_{3}\right) \\
& \left.+f\left(x_{1}, x_{2}, x_{3}\right)\right]+\left[f\left(x_{1}-u_{1}, x_{2}, x_{3}\right)-f\left(x_{1}, x_{2}, x_{3}\right)\right] \\
& +\left[f\left(x_{1}, x_{2}-u_{2}, x_{3}-u_{3}\right)-f\left(x_{1}, x_{2}-u_{2}, x_{3}\right)\right] \\
& +\left[f\left(x_{1}, x_{2}-u_{2}, x_{3}\right)-f\left(x_{1}, x_{2}, x_{3}\right)\right]+f\left(x_{1}, x_{2}, x_{3}\right) .
\end{aligned}
$$

into the first term

$$
\int_{\epsilon_{1} \leq\left|u_{1}\right| \leq 1} \int_{\epsilon_{2} \leq\left|u_{2}\right| \leq 1} \int_{\epsilon_{3} \leq\left|u_{3}\right| \leq 1} \mathcal{K}\left(u_{1}, u_{2}, u_{3}\right) f\left(x_{1}-u_{1}, x_{2}-u_{2}, x_{3}-u_{3}\right) \mathrm{d} u_{1} \mathrm{~d} u_{2} \mathrm{~d} u_{3}
$$

yields five integrals. In view of the conditions of $f$ and the condition (RR) on $\mathcal{K}$, the first integral is dominated by

$$
F_{1}\left(x_{1}\right) F_{2}\left(x_{2}\right) F_{3}\left(x_{3}\right) \int_{\left|u_{1}\right| \leq 1} \int_{\left|u_{2}\right| \leq 1} \int_{\left|u_{3}\right| \leq 1} \frac{1}{\left|u_{1}\right|\left|u_{2}\right|\left|u_{3}\right|} \frac{\left|u_{1}\right|\left(\left|u_{2}\right|+\left|u_{3}\right|\right)}{\left(\left|\frac{u_{1} u_{2}}{u_{3}}\right|+\left|\frac{u_{3}}{u_{1} u_{2}}\right|\right)^{\theta_{2}}} \mathrm{~d} u_{1} \mathrm{~d} u_{2} \mathrm{~d} u_{3},
$$


where $F_{1}\left(x_{1}\right), F_{2}\left(x_{2}\right)$, and $F_{3}\left(x_{3}\right)$ are bounded functions with bounded supports. Thus, as $\epsilon_{1}, \epsilon_{2}, \epsilon_{3} \rightarrow 0$, the limit of the first integral exists for each $x_{1}, x_{2}$, and $x_{3}$ and, moreover, is dominated by a fixed bounded function with compact support. Therefore, the first integral converges in $L^{2}$ as $\epsilon_{1}, \epsilon_{2}, \epsilon_{3} \rightarrow 0$. The third integral can be handled by the same way. To see the second integral, by the condition (C1.c) and the assumption on $\mathcal{K}$ we observe that the limit $\int_{\epsilon_{2} \leq\left|u_{2}\right| \leq 1} \int_{\epsilon_{3} \leq\left|u_{3}\right| \leq 1} \mathcal{K}\left(u_{1}, u_{2}, u_{3}\right) \mathrm{d} u_{2} \mathrm{~d} u_{3}$ exists as $\epsilon_{2}, \epsilon_{3} \rightarrow 0$, and is dominated by $C\left|u_{1}\right|^{-1}$. This fact together with the smoothness condition on $f$ implies the second integral converges in $L^{2}$ as $\epsilon_{1}, \epsilon_{2}, \epsilon_{3} \rightarrow 0$ and the limit is dominated by a bounded function with compact support. Similarly, the required results for the fourth and the last integrals follow from the conditions (C1.d) and (C1.a), respectively, together with the assumptions on $\mathcal{K}$.

Note that in fact $\mathcal{K}(u)$ is integrable over the sets $(i i)\left|u_{1}\right| \leq 1,\left|u_{2}\right| \leq 1,\left|u_{3}\right| \geq 1$ and (vii) $\left|u_{1}\right| \geq 1,\left|u_{2}\right| \geq 1,\left|u_{3}\right| \leq 1$. Thus we have all the required results over these two sets.

Observe that

$$
\begin{aligned}
\int_{\mathbb{R}} & \int_{\mathbb{R}} \int_{\left|u_{3}\right| \geq 1}|\mathcal{K}(u) f(x-u)| d u \\
& \lesssim \int_{\mathbb{R}} \int_{\mathbb{R}} \int_{\left|u_{3}\right| \geq 1} \frac{1}{\left|u_{1}\right|\left|u_{2}\right|\left|u_{3}\right|}\left(\left|\frac{u_{1} u_{2}}{u_{3}}\right|+\left|\frac{u_{3}}{u_{1} u_{2}}\right|\right)^{-\theta_{2}} \\
& \times \frac{1}{\left(1+\left|x_{1}-u_{1}\right|\right)^{2}\left(1+\left|x_{2}-u_{2}\right|\right)^{2}\left(1+\left|x_{3}-u_{3}\right|\right)^{2}} d u
\end{aligned}
$$

which belongs to $L^{2}\left(\mathbb{R}^{3}\right)$. This implies the required results over the corresponding sets (iv), (vi), and (viii).

To handle the integral over the set (iii) $\left|u_{1}\right| \leq 1,\left|u_{2}\right| \geq 1,\left|u_{3}\right| \leq 1$, inserting

$$
\begin{aligned}
f\left(x_{1}-u_{1}, x_{2}-u_{2}, x_{3}-u_{3}\right)= & {\left[f\left(x_{1}-u_{1}, x_{2}-u_{2}, x_{3}-u_{3}\right)\right.} \\
& \left.-f\left(x_{1}, x_{2}-u_{2}, x_{3}-u_{3}\right)\right] \\
& +\left[f\left(x_{1}, x_{2}-u_{2}, x_{3}-u_{3}\right)-f\left(x_{1}, x_{2}-u_{2}, x_{3}\right)\right] \\
& +f\left(x_{1}, x_{2}-u_{2}, x_{3}\right)
\end{aligned}
$$

yields three integrals over the set (iii). The first two integrals, by the condition (RR) and the smoothness of $f$, are dominated by

$$
\begin{aligned}
& F_{1}\left(x_{1}\right) F_{3}\left(x_{3}\right) \int_{\left|u_{1}\right| \leq 1} \int_{\left|u_{2}\right| \geq 1} \int_{\left|u_{3}\right| \leq 1} \frac{1}{\left|u_{2}\right|\left|u_{3}\right|}\left(\left|\frac{u_{1} u_{2}}{u_{3}}\right|+\left|\frac{u_{3}}{u_{1} u_{2}}\right|\right)^{-\theta_{2}} \\
& \frac{1}{\left(1+\left|x_{2}-u_{2}\right|\right)^{2}} \mathrm{~d} u_{1} \mathrm{~d} u_{2} \mathrm{~d} u_{3}
\end{aligned}
$$


and

$$
\begin{aligned}
& F_{1}\left(x_{1}\right) F_{3}\left(x_{3}\right) \int_{\left|u_{1}\right| \leq 1} \int_{\left|u_{2}\right| \geq 1} \int_{\left|u_{3}\right| \leq 1} \frac{1}{\left|u_{1}\right|\left|u_{2}\right|}\left(\left|\frac{u_{1} u_{2}}{u_{3}}\right|+\left|\frac{u_{3}}{u_{1} u_{2}}\right|\right)^{-\theta_{2}} \\
& \times \frac{1}{\left(1+\left|x_{2}-u_{2}\right|\right)^{2}} \mathrm{~d} u_{1} \mathrm{~d} u_{2} \mathrm{~d} u_{3},
\end{aligned}
$$

where $F_{1}\left(x_{1}\right)$ and $F_{3}\left(x_{3}\right)$ are bounded functions with bounded supports. Thus, we obtain a domination, independent of $\epsilon_{1}, \epsilon_{3}$ and $N_{2}$, by a function which belongs to $L^{2}\left(\mathbb{R}^{3}\right)$, so the limits as $\epsilon_{1}, \epsilon_{3} \rightarrow 0$ and $N_{2} \rightarrow \infty$ exist. Applying condition (C1.d) with $\beta=0$ yields that the last integral is bounded by

$$
F_{1}\left(x_{1}\right) F_{3}\left(x_{3}\right) \int_{\left|u_{2}\right| \geq 1} \frac{1}{\left|u_{2}\right|\left(1+\left|x_{2}-u_{2}\right|\right)^{2}} \mathrm{~d} u_{2}
$$

which belongs to $L^{2}\left(\mathbb{R}^{3}\right)$ and the limit as $\epsilon_{1}, \epsilon_{3} \rightarrow 0$ and $N_{2} \rightarrow \infty$ exists.

Finally, for the integral over the set $(v)\left|u_{1}\right| \geq 1,\left|u_{2}\right| \leq 1,\left|u_{3}\right| \leq 1$, inserting

$$
\begin{aligned}
f\left(x_{1}-u_{1}, x_{2}-u_{2}, x_{3}-u_{3}\right)= & {\left[f\left(x_{1}-u_{1}, x_{2}-u_{2}, x_{3}-u_{3}\right)\right.} \\
& \left.-f\left(x_{1}-u_{1}, x_{2}-u_{2}, x_{3}\right)\right] \\
+ & {\left[f\left(x_{1}-u_{1}, x_{2}-u_{2}, x_{3}\right)-f\left(x_{1}-u_{1}, x_{2}, x_{3}\right)\right] } \\
& +f\left(x_{1}-u_{1}, x_{2}, x_{3}\right)
\end{aligned}
$$

and then applying the condition (RR) for the first two integrals and (C1.c) with $\alpha=0$ on the last integral, this integral is dominated by

$$
F_{2}\left(x_{2}\right) F_{3}\left(x_{3}\right) \int_{\left|u_{1}\right| \geq 1} \frac{1}{\left|u_{1}\right|\left(1+\left|x_{1}-u_{1}\right|\right)^{2}} \mathrm{~d} u_{1}
$$

where $F_{2}\left(x_{2}\right)$ and $F_{3}\left(x_{3}\right)$ are bounded functions with bounded supports. The existence of the limit is concluded. The $L^{2}$ boundedness of $\mathcal{K} * f$ then follows from Theorem 1.1.

We would like to remark, as mentioned early in Sect. 1, that incidentally we have shown that $\mathcal{K}_{\epsilon}^{N} * f$ converges in $L^{p}$ norm and almost everywhere as $\epsilon_{1}, \epsilon_{2}, \epsilon_{3} \rightarrow 0$ and $N_{1}, N_{2}, N_{3} \rightarrow \infty$ whenever $f$ is a smooth function with compact support. We also point out that the condition (C1.b) is not used in the proof of Corollary 1.2.

\section{$3 L^{p}$ Estimates for $1<p<\infty$}

In this subsection, we will prove Theorems 1.3 and 1.4. The main tools to show the $L^{p}, 1<p<\infty$, estimates are 
- the $L^{2}$ boundedness of $\mathcal{K} * f$,

- the Littlewood-Paley theory associated with Zygmund dilation,

- the almost orthogonality argument.

We first recall the Littlewood-Paley theory. As mentioned in Sect. 1, to handle the $L^{p}, 1<p<\infty$, boundedness of operators, one only needs the continuous Littlewood-Paley square function. To do this, let $\mathcal{S}\left(\mathbb{R}^{i}\right)$ denote the Schwartz class in $\mathbb{R}^{i}, i=1,2,3$. We construct a function defined on $\mathbb{R}^{3}$ given by

$$
\phi\left(x_{1}, x_{2}, x_{3}\right)=\phi^{(1)}(x) \phi^{(2)}\left(x_{2}, x_{3}\right),
$$

where $\phi^{(1)} \in \mathcal{S}(\mathbb{R}), \phi^{(2)} \in \mathcal{S}\left(\mathbb{R}^{2}\right)$ with the supports contained in the unit ball centered at the origin in $\mathbb{R}^{3}$, and satisfy

$$
\begin{gathered}
\left.\sum_{j \in \mathbb{Z}} \widehat{\mid \phi^{(1)}}\left(2^{j} \xi_{1}\right)\right|^{2}=1 \text { for all } \xi_{1} \in \mathbb{R} \backslash\{0\}, \\
\left.\sum_{k \in \mathbb{Z}} \widehat{\mid \phi^{(2)}}\left(2^{k} \xi_{2}, 2^{k} \xi_{3}\right)\right|^{2}=1 \text { for all }\left(\xi_{2}, \xi_{3}\right) \in \mathbb{R}^{2} \backslash\{(0,0)\},
\end{gathered}
$$

and the moment conditions

$$
\int_{\mathbb{R}} x_{1}^{\alpha} \phi^{(1)}\left(x_{1}\right) \mathrm{d} x_{1}=\int_{\mathbb{R}^{2}} x_{2}^{\beta} x_{3}^{\gamma} \phi^{(2)}\left(x_{2}, x_{3}\right) \mathrm{d} x_{2} \mathrm{~d} x_{3}=0 \quad \text { for } 0 \leq \alpha, \beta, \gamma \leq 10 .
$$

For $f \in L^{p}, 1<p<\infty, g_{\mathfrak{z}}^{c}(f)$, the continuous Littlewood-Paley square function of $f$ associated with the Zygmund dilation is defined by

$$
g_{\mathfrak{z}}^{c}(f)(x)=\left\{\sum_{j, k \in \mathbb{Z}}\left|\left(\phi_{j, k} * f\right)(x)\right|^{2}\right\}^{\frac{1}{2}}
$$

where

$$
\phi_{j, k}\left(x_{1}, x_{2}, x_{3}\right):=2^{-2(j+k)} \phi^{(1)}\left(2^{-j} x_{1}\right) \phi^{(2)}\left(2^{-k} x_{2}, 2^{-(j+k)} x_{3}\right) .
$$

By taking the Fourier transform, it is easy to see that the following Calderón's identity

$$
f(x)=\sum_{j, k \in \mathbb{Z}}\left(\phi_{j, k} * \phi_{j, k} * f\right)(x)
$$

holds on $L^{2}\left(\mathbb{R}^{3}\right)$.

Using the $L^{p}$ boundedness of operators for $1<p<\infty$ obtained by Ricci and Stein in [28], as mentioned in Sect. 1, we have

$$
\left\|\sum_{(j, k) \in F} \epsilon(j, k)\left(\phi_{j, k} * f\right)\right\|_{p} \leq C\|f\|_{p}
$$


for every sequence $\epsilon(j, k)$, taking the values 1 and -1 , where $F$ is any finite subset of $j, k \in \mathbb{Z}$. Using Khinchin's well-known inequality, we obtain

$$
\left\|g_{\mathfrak{z}}^{c}(f)\right\|_{p} \leq C_{2}\|f\|_{p}
$$

for $1<p<\infty$. This estimate together with Calderón's identity on $L^{2}$ allows us to obtain the $L^{p}$ estimates of $g_{\mathfrak{z}}^{c}$ for $1<p<\infty$. Namely, there exist constants $C_{1}$ and $C_{2}$ such that, for $1<p<\infty$,

$$
C_{1}\|f\|_{p} \leq\left\|g_{\mathfrak{z}}^{c}(f)\right\|_{p} \leq C_{2}\|f\|_{p} .
$$

Now we turn to the proof of Theorem 1.3. First note that $\mathcal{K}$ satisfies the conditions (C1.a)-(C1.d) since (C2.b) implies (C1.b) and (C1.d), as mentioned in Sect. 1. Therefore, by Corollary 1.2, the operator $\mathcal{K} * f=\lim _{\substack{\epsilon_{1}, \epsilon_{2}, \epsilon_{3} \rightarrow 0 \\ N_{1}, N_{2}, N_{3} \rightarrow \infty}} \mathcal{K}_{\epsilon}^{N} * f$ is bounded on $L^{2}\left(\mathbb{R}^{3}\right)$. To obtain the $L^{p}$ boundedness of $\mathcal{K} * f$, it suffices to show this for all $f \in L^{2} \cap L^{p}$ since the subspace $L^{2} \cap L^{p}$ is dense in $L^{p}, 1<p<\infty$. By the $L^{p}$ estimates of the Littlewood-Paley square function given in (3.7), the $L^{p}$ boundedness of $\mathcal{K} * f$ will follow from the estimate

$$
\left\|g_{\mathfrak{z}}^{c}(\mathcal{K} * f)\right\|_{p} \lesssim\|f\|_{p}
$$

To prove (3.8) for all $f \in L^{2} \cap L^{p}$, using the fact that $\mathcal{K} * f$ is bounded on $L^{2}$, as mentioned above, and Calderón's identity on $L^{2}$ given in (3.6), we write

$$
\phi_{j, k} *(\mathcal{K} * f)\left(x_{1}, x_{2}, x_{3}\right)=\sum_{j^{\prime}, k^{\prime} \in \mathbb{Z}}\left(\phi_{j, k} * \mathcal{K} * \phi_{j^{\prime}, k^{\prime}} * \phi_{j^{\prime}, k^{\prime}} * f\right)\left(x_{1}, x_{2}, x_{3}\right)
$$

The proof of Theorem 1.3 now follows from the following almost orthogonality argument.

Proposition 3.1 Suppose that $\phi$ is defined as in (3.5) and $\mathcal{K}$ is a function on $\mathbb{R}^{3}$ satisfying the conditions (RR) and (C2.a)-(C2.c). Then, for $\lambda=\frac{1}{2} \min \left(\theta_{1}, \theta_{2}\right)$,

$$
\begin{aligned}
& \left|\left(\phi_{j, k} * \mathcal{K} * \phi_{j^{\prime}, k^{\prime}}\right)\left(x_{1}, x_{2}, x_{3}\right)\right| \\
& \quad \leq C 2^{-\left|j-j^{\prime}\right|} 2^{-\left|k-k^{\prime}\right|} \frac{2^{-\left(j \vee j^{\prime}\right)}}{\left(1+2^{-\left(j \vee j^{\prime}\right)}\left|x_{1}\right|\right)^{1+\lambda}} \\
& \quad \times \frac{2^{-\left(k \vee k^{\prime}\right)}}{\left(1+2^{-\left(k \vee k^{\prime}\right)}\left|x_{2}\right|\right)^{1+\lambda}} \frac{2^{-\left(j \vee j^{\prime}\right)-\left(k \vee k^{\prime}\right)}}{\left(1+2^{-\left(j \vee j^{\prime}\right)-\left(k \vee k^{\prime}\right)}\left|x_{3}\right|\right)^{1+\lambda}},
\end{aligned}
$$

where the constant $C$ depends only on $\lambda$ and $\mathcal{K} * f$ is defined for $f \in L^{2}$ as in Corollary 1.2, and $j \vee j^{\prime}$ means $\max \left(j, j^{\prime}\right)$. 
Assuming Proposition 3.1 for the moment, we then observe that

$$
\begin{aligned}
& \left|\left[\phi_{j, k} * \mathcal{K} * \phi_{j^{\prime}, k^{\prime}} *\left(\phi_{j^{\prime}, k^{\prime}} * f\right)\right]\left(x_{1}, x_{2}, x_{3}\right)\right| \\
& \quad \leq C 2^{-\left|j-j^{\prime}\right|} 2^{-\left|k-k^{\prime}\right|} \mathcal{M}_{s}\left(\phi_{j^{\prime}, k^{\prime}} * f\right)\left(x_{1}, x_{2}, x_{3}\right),
\end{aligned}
$$

where $\mathcal{M}_{s}$ is the strong maximal function on $\mathbb{R}^{3}$. Hölder's inequality implies

$$
\begin{aligned}
\left\|g_{\mathfrak{z}}^{c}(\mathcal{K} * f)\right\|_{p} & =\left\|\left\{\sum_{j, k}\left|\phi_{j, k} * \mathcal{K} * f\right|^{2}\right\}^{\frac{1}{2}}\right\|_{p} \leq C\left\|\left\{\sum_{j^{\prime}, k^{\prime}}\left|\mathcal{M}_{s}\left(\phi_{j^{\prime}, k^{\prime}} * f\right)\right|^{2}\right\}^{\frac{1}{2}}\right\|_{p} \\
& \leq C\left\|\left\{\sum_{j^{\prime}, k^{\prime}}\left|\phi_{j^{\prime}, k^{\prime}} * f\right|^{2}\right\}^{\frac{1}{2}}\right\|_{p} \leq C\|f\|_{p},
\end{aligned}
$$

where we use the Fefferman and Stein's vector-valued maximal inequality and the Littlewood-Paley square function estimate for $L^{p}, 1<p<\infty$, in the last two inequalities, respectively.

To finish the proof of Theorem 1.3, we only need to show Proposition 3.1, whose proof follows from the following lemma.

Lemma 3.2 Suppose that $\phi^{(1)}$ and $\phi^{(2)}$ satisfy the conditions (3.1)-(3.4) and $\mathcal{K}$ is a function on $\mathbb{R}^{3}$ satisfying the conditions (RR) and (C2.a)-(C2.c). Then, for $\lambda=$ $\frac{1}{2} \min \left(\theta_{1}, \theta_{2}\right)$, we have

$$
\left|\mathcal{K} *\left(\phi^{(1)} \otimes \phi^{(2)}\right)\left(x_{1}, x_{2}, x_{3}\right)\right| \leq \frac{C_{\lambda}}{\left(1+\left|x_{1}\right|\right)^{1+\lambda}\left(1+\left|x_{2}\right|\right)^{1+\lambda}\left(1+\left|x_{3}\right|\right)^{1+\lambda}}
$$

where $C_{\lambda}$ is the constant depending only on $\lambda$.

Proof For simplicity, let $S=\lim _{\substack{\epsilon_{1}, \epsilon_{2}, \epsilon_{3} \rightarrow 0 \\ N_{1}, N_{2}, N_{3} \rightarrow \infty}} \int_{\epsilon_{1} \leq|x-u| \leq N_{1}} \int_{\epsilon_{2} \leq\left|x_{2}-u_{2}\right| \leq N_{2}}$ $\int_{\epsilon_{3} \leq\left|x_{3}-u_{3}\right| \leq N_{3}} \mathcal{K}\left(x_{1}-u_{1}, x_{2}-u_{2}, x_{3}-u_{3}\right) \phi^{(1)}\left(u_{1}\right) \phi^{(2)}\left(u_{2}, u_{3}\right) \mathrm{d} u_{3} \mathrm{~d} u_{2} \mathrm{~d} u_{1}$. We consider the following eight cases:

Case $1\left|x_{1}\right| \geq 3,\left|x_{2}\right| \geq 3,\left|x_{3}\right| \geq 3$. For this case, we use the cancellation conditions in (3.4) to write

$$
\begin{aligned}
S= & \lim _{\substack{\epsilon_{1}, \epsilon_{2}, \epsilon_{3} \rightarrow 0 \\
N_{1}, N_{2}, N_{3} \rightarrow \infty}} \int_{\epsilon_{1} \leq\left|x_{1}-u_{1}\right| \leq N_{1}} \int_{\epsilon_{2} \leq\left|x_{2}-u_{2}\right| \leq N_{2}} \int_{\epsilon_{3} \leq\left|x_{3}-u_{3}\right| \leq N_{3}} \\
& \times\left[\mathcal{K}\left(x_{1}-u_{1}, x_{2}-u_{2}, x_{3}-u_{3}\right)\right. \\
& \left.-\mathcal{K}\left(x_{1}, x_{2}-u_{2}, x_{3}-u_{3}\right)-\mathcal{K}\left(x_{1}-u_{1}, x_{2}, x_{3}\right)+\mathcal{K}\left(x_{1}, x_{2}, x_{3}\right)\right] \\
& \times \phi^{(1)}\left(u_{1}\right) \phi^{(2)}\left(u_{2}, u_{3}\right) \mathrm{d} u_{3} \mathrm{~d} u_{2} \mathrm{~d} u_{1} .
\end{aligned}
$$

Note that $\mathcal{K}\left(x_{1}-u_{1}, x_{2}-u_{2}, x_{3}-u_{3}\right)-\mathcal{K}\left(x_{1}, x_{2}-u_{2}, x_{3}-u_{3}\right)-\left(\mathcal{K}\left(x_{1}-u_{1}, x_{2}, x_{3}\right)-\right.$ $\left.\mathcal{K}\left(x_{1}, x_{2}, x_{3}\right)\right)=\Delta_{x_{2},-u_{2}} \Delta_{x_{1},-u_{1}} \mathcal{K}\left(x_{1}, x_{2}, x_{3}-u_{3}\right)+\Delta_{x_{3},-u_{3}} \Delta_{x_{1},-u_{1}} \mathcal{K}\left(x_{1}, x_{2}, x_{3}\right)$. 
Thus, by the condition (RR) with $\alpha=\beta=1, \gamma=0$ and $\alpha=\gamma=1, \beta=0$ respectively, we have

$$
\begin{aligned}
|S| & \lesssim \int_{\left|u_{1}\right| \leq 1} \int_{\left|u_{2}\right| \leq 1} \int_{\left|u_{3}\right| \leq 1} \frac{\left|u_{1}\right|^{\theta_{1}}\left(\left|u_{2}\right|^{\theta_{1}}+\left|u_{3}\right|^{\theta_{1}}\right)}{\left|x_{1}\right|^{1+\theta_{1}\left|x_{2}\right|^{1+\theta_{1}}\left|x_{3}\right|}}\left(\left|\frac{x_{1} x_{2}}{x_{3}}\right|+\left|\frac{x_{3}}{x_{1} x_{2}}\right|\right)^{-\lambda} \mathrm{d} u_{3} \mathrm{~d} u_{2} \mathrm{~d} u_{1} \\
& \lesssim \frac{1}{\left(1+\left|x_{1}\right|\right)^{1+\lambda}\left(1+\left|x_{2}\right|\right)^{1+\lambda}\left(1+\left|x_{3}\right|\right)^{1+\lambda}} .
\end{aligned}
$$

Case $2\left|x_{1}\right| \geq 3,\left|x_{2}\right| \geq 3,\left|x_{3}\right|<3$. By the cancellation condition of $\phi^{(1)}$,

$$
\begin{aligned}
S= & \lim _{\substack{\epsilon_{1}, \epsilon_{2}, \epsilon_{3} \rightarrow 0 \\
N_{1}, N_{2}, N_{3} \rightarrow \infty}} \int_{\epsilon_{1} \leq\left|x_{1}-u_{1}\right| \leq N_{1}} \int_{\epsilon_{2} \leq\left|x_{2}-u_{2}\right| \leq N_{2}} \int_{\epsilon_{3} \leq\left|x_{3}-u_{3}\right| \leq N_{3}} \\
& \times \Delta_{x_{1},-u_{1}} \mathcal{K}\left(x_{1}, x_{2}-u_{2}, x_{3}-u_{3}\right) \times \phi^{(1)}\left(u_{1}\right) \phi^{(2)}\left(u_{2}, u_{3}\right) \mathrm{d} u_{3} \mathrm{~d} u_{2} \mathrm{~d} u_{1} .
\end{aligned}
$$

Therefore, by the condition (RR) with $\alpha=1$ and $\beta=\gamma=0$, we obtain

$$
\begin{aligned}
& |S| \lesssim \int_{\left|u_{1}\right| \leq 1} \int_{\left|u_{2}\right| \leq 1} \int_{\left|u_{3}\right| \leq 1} \frac{\left|u_{1}\right|^{\theta_{1}}}{\left|x_{1}\right|^{1+\theta_{1}}\left|x_{2}-u_{2}\right|\left|x_{3}-u_{3}\right|} \\
& \times\left(\left|\frac{x_{1}\left(x_{2}-u_{2}\right)}{x_{3}-u_{3}}\right|+\left|\frac{x_{3}-u_{3}}{x_{1}\left(x_{2}-u_{2}\right)}\right|\right)^{-\lambda} \mathrm{d} u_{3} \mathrm{~d} u_{2} \mathrm{~d} u_{1} \\
& \lesssim \int_{\left|u_{1}\right| \leq 1} \int_{\left|u_{2}\right| \leq 1} \int_{\left|u_{3}\right| \leq 1} \frac{1}{\left|x_{1}\right|^{1+\theta_{1}}\left|x_{2}\right|\left|x_{3}-u_{3}\right|}\left|\frac{x_{1} x_{2}}{x_{3}-u_{3}}\right|^{-\lambda} \mathrm{d} u_{3} \mathrm{~d} u_{2} \mathrm{~d} u_{1} \\
& \lesssim \frac{1}{\left(1+\left|x_{1}\right|\right)^{1+\lambda}\left(1+\left|x_{2}\right|\right)^{1+\lambda}\left(1+\left|x_{3}\right|\right)^{1+\lambda}} .
\end{aligned}
$$

Case $3\left|x_{1}\right| \geq 3,\left|x_{2}\right|<3,\left|x_{3}\right| \geq 3$. The same expression for $S$ as in Case 2 yields

$$
\begin{aligned}
|S| & \lesssim \int_{\left|u_{1}\right| \leq 1} \int_{\left|u_{2}\right| \leq 1} \int_{\left|u_{3}\right| \leq 1} \frac{\left|u_{1}\right|^{\theta_{1}}}{\left|x_{1}\right|^{1+\theta_{1}\left|x_{2}-u_{2}\right|\left|x_{3}\right|}}\left|\frac{x_{3}}{x_{1}\left(x_{2}-u_{2}\right)}\right|^{-\lambda} \mathrm{d} u_{3} \mathrm{~d} u_{2} \mathrm{~d} u_{1} \\
& \lesssim \frac{1}{\left(1+\left|x_{1}\right|\right)^{1+\lambda}\left(1+\left|x_{2}\right|\right)^{1+\lambda}\left(1+\left|x_{3}\right|\right)^{1+\lambda}} .
\end{aligned}
$$

Case $4\left|x_{1}\right| \geq 3,\left|x_{2}\right|<3,\left|x_{3}\right|<3$. Using the cancellation condition of $\phi^{(1)}$, we write

$$
\begin{aligned}
S= & \lim _{\substack{\epsilon_{1}, \epsilon_{2}, \epsilon_{3} \rightarrow 0 \\
N_{1}, N_{2}, N_{3} \rightarrow \infty}} \int_{\epsilon_{1} \leq\left|x_{1}-u_{1}\right| \leq N_{1}} \int_{\epsilon_{2} \leq\left|u_{2}\right| \leq 4} \int_{\epsilon_{3} \leq\left|u_{3}\right| \leq 4} \\
& \times\left[\mathcal{K}\left(x_{1}-u_{1}, u_{2}, u_{3}\right)-\mathcal{K}\left(x_{1}, u_{2}, u_{3}\right)\right] \\
& \times \phi^{(1)}\left(u_{1}\right)\left(\phi^{(2)}\left(x_{2}-u_{2}, x_{3}-u_{3}\right)-\phi^{(2)}\left(x_{2}, x_{3}\right)\right) \mathrm{d} u_{3} \mathrm{~d} u_{2} \mathrm{~d} u_{1}
\end{aligned}
$$




$$
\begin{aligned}
& +\lim _{\substack{\epsilon_{1}, \epsilon_{2}, \epsilon_{3} \rightarrow 0 \\
N_{1}, N_{2}, N_{3} \rightarrow \infty}} \int_{\epsilon_{1} \leq\left|x_{1}-u_{1}\right| \leq N_{1}} \int_{\epsilon_{2} \leq\left|u_{2}\right| \leq 4} \int_{\epsilon_{3} \leq\left|u_{3}\right| \leq 4} \\
& \times\left[\mathcal{K}\left(x_{1}-u_{1}, u_{2}, u_{3}\right)-\mathcal{K}\left(x_{1}, u_{2}, u_{3}\right)\right] \phi^{(1)}\left(u_{1}\right) \phi^{(2)}\left(x_{2}, x_{3}\right) \mathrm{d} u_{3} \mathrm{~d} u_{2} \mathrm{~d} u_{1} .
\end{aligned}
$$

By the condition (RR) with $\alpha=1, \beta=\gamma=0$ for the first integral, and the cancellation condition (C2.c) with $\alpha=1$ for the second integral, we get

$$
\begin{aligned}
|S| \lesssim & \int_{\left|u_{1}\right| \leq 1} \int_{\left|u_{2}\right| \leq 4} \int_{\left|u_{3}\right| \leq 4} \frac{\left|u_{1}\right|^{\theta_{1}}}{\left|x_{1}\right|^{1+\theta_{1}\left|u_{2}\right|\left|u_{3}\right|}}\left(\left|\frac{x_{1} u_{2}}{u_{3}}\right|+\left|\frac{u_{3}}{x_{1} u_{2}}\right|\right)^{-\theta_{2}} \\
& \left(\left|u_{2}\right|+\left|u_{3}\right|\right) \mathrm{d} u_{3} \mathrm{~d} u_{2} \mathrm{~d} u_{1} \\
& +\int_{\left|u_{1}\right| \leq 1} \frac{\left|u_{1}\right|^{\theta_{1}}}{\left|x_{1}\right|^{1+\theta_{1}}} \mathrm{~d} u_{1} \\
& \lesssim \frac{1}{\left|x_{1}\right|^{1+\theta_{1}}} \\
& \lesssim \frac{1}{\left(1+\left|x_{1}\right|\right)^{1+\lambda}\left(1+\left|x_{2}\right|\right)^{1+\lambda}\left(1+\left|x_{3}\right|\right)^{1+\lambda}} .
\end{aligned}
$$

Case $5\left|x_{1}\right|<3,\left|x_{2}\right| \geq 3,\left|x_{3}\right| \geq 3$. Similar to Case 4, using the cancellation condition of $\phi^{(2)}$, we write

$$
\begin{aligned}
S= & \lim _{\substack{\epsilon_{1}, \epsilon_{2}, \epsilon_{3} \rightarrow 0 \\
N_{1}, N_{2}, N_{3} \rightarrow \infty}} \int_{\epsilon_{1} \leq\left|u_{1}\right| \leq 4} \int_{\epsilon_{2} \leq\left|x_{2}-u_{2}\right| \leq N_{2}}\left[\mathcal{K}\left(u_{1}, x_{2}-u_{2}, x_{3}-u_{3}\right)-\mathcal{K}\left(u_{1}, x_{2}, x_{3}\right)\right] \\
& \times \int_{\epsilon_{3} \leq\left|x_{3}-u_{3}\right| \leq N_{3}} \\
& \times\left(\phi^{(1)}\left(x_{1}-u_{1}\right)-\phi^{(1)}\left(x_{1}\right)\right) \phi^{(2)}\left(u_{2}, u_{3}\right) \mathrm{d} u_{3} \mathrm{~d} u_{2} \mathrm{~d} u_{1} \\
& +\lim _{\substack{\epsilon_{1}, \epsilon_{2}, \epsilon_{3} \rightarrow 0 \\
N_{1}, N_{2}, N_{3} \rightarrow \infty}} \int_{\epsilon_{1} \leq\left|u_{1}\right| \leq 4} \int_{\epsilon_{2} \leq\left|x_{2}-u_{2}\right| \leq N_{2}}\left[\mathcal{K}\left(u_{1}, x_{2}-u_{2}, x_{3}-u_{3}\right)-\mathcal{K}\left(u_{1}, x_{2}, x_{3}\right)\right] \\
& \times \int_{\epsilon_{3} \leq\left|x_{3}-u_{3}\right| \leq N_{3}}\left[\begin{array}{l}
(1)\left(x_{1}\right) \phi^{(2)}\left(u_{2}, u_{3}\right) \mathrm{d} u_{3} \mathrm{~d} u_{2} \mathrm{~d} u_{1} . \\
\end{array}\right.
\end{aligned}
$$

Note that $\mathcal{K}\left(u_{1}, x_{2}-u_{2}, x_{3}-u_{3}\right)-\mathcal{K}\left(u_{1}, x_{2}, x_{3}\right)=\Delta_{x_{2},-u_{2}} \mathcal{K}\left(u_{1}, x_{2}, x_{3}\right)+$ $\Delta_{x_{3},-u_{3}} \mathcal{K}\left(u_{1}, x_{2}, x_{3}\right)$ and $\mathcal{K}\left(u_{1}, x_{2}-u_{2}, x_{3}-u_{3}\right)-\mathcal{K}\left(u_{1}, x_{2}, x_{3}\right)=\Delta_{x_{2},-u_{2}}$ $\mathcal{K}\left(u_{1}, x_{2}, x_{3}-u_{3}\right)+\Delta_{x_{3},-u_{3}} \mathcal{K}\left(u_{1}, x_{2}, x_{3}\right)$. Thus, using the condition (RR) on $\mathcal{K}$, the smoothness of $\phi^{(1)}$ for the first integral, and the cancellation conditions (C2.b) with $\beta=1, \gamma=0$ and $\beta=0, \gamma=1$, respectively, for the second integral, and applying the dominated convergence theorem, we obtain 


$$
\begin{aligned}
|S| \lesssim & \int_{\left|u_{1}\right| \leq 4} \int_{\left|u_{2}\right| \leq 1} \int_{\left|u_{3}\right| \leq 1}\left(\frac{\left|u_{2}\right|^{\theta_{1}}}{\left|u_{1}\right|\left|x_{2}\right|^{1+\theta_{1}}\left|x_{3}\right|}+\frac{\left|u_{3}\right|^{\theta_{1}}}{\left|u_{1}\right|\left|x_{2}\right|\left|x_{3}\right|^{1+\theta_{1}}}\right) \\
& \times\left(\left|\frac{u_{1} x_{2}}{x_{3}}\right|+\left|\frac{x_{3}}{u_{1} x_{2}}\right|\right)^{-\lambda}\left|u_{1}\right| \mathrm{d} u_{3} \mathrm{~d} u_{2} \mathrm{~d} u_{1} \\
& +\int_{\left|u_{2}\right| \leq 1} \int_{\left|u_{3}\right| \leq 1}\left(\frac{\left|u_{2}\right|^{\theta_{1}}}{\left|x_{2}\right|^{1+\theta_{1}\left|x_{3}\right|}}+\frac{\left|u_{3}\right|^{\theta_{1}}}{\left|x_{2}\right|\left|x_{3}\right|^{1+\theta_{1}}}\right)\left(\left|\frac{4 x_{2}}{x_{3}}\right|+\left|\frac{x_{3}}{4 x_{2}}\right|\right)^{-\lambda} \mathrm{d} u_{3} \mathrm{~d} u_{2} \\
\lesssim & \frac{1}{\left(1+\left|x_{1}\right|\right)^{1+\lambda}\left(1+\left|x_{2}\right|\right)^{1+\lambda}\left(1+\left|x_{3}\right|\right)^{1+\lambda}} .
\end{aligned}
$$

Case $6\left|x_{1}\right|<3,\left|x_{2}\right| \geq 3,\left|x_{3}\right|<3$. Note that

$$
\begin{aligned}
S= & \lim _{\substack{\epsilon_{1}, \epsilon_{2}, \epsilon_{3} \rightarrow 0 \\
N_{1}, N_{2}, N_{3} \rightarrow \infty}} \int_{\epsilon_{1} \leq\left|u_{1}\right| \leq 4} \int_{\epsilon_{2} \leq\left|x_{2}-u_{2}\right| \leq N_{2}} \int_{\epsilon_{3} \leq\left|x_{3}-u_{3}\right| \leq N_{3}} \mathcal{K}\left(u_{1}, x_{2}-u_{2}, x_{3}-u_{3}\right) \\
& \times\left(\phi^{(1)}\left(x_{1}-u_{1}\right)-\phi^{(1)}\left(x_{1}\right)\right) \phi^{(2)}\left(u_{2}, u_{3}\right) \mathrm{d} u_{3} \mathrm{~d} u_{2} \mathrm{~d} u_{1} \\
& +\lim _{\substack{\epsilon_{1}, \epsilon_{2}, \epsilon_{3} \rightarrow 0 \\
N_{1}, N_{2}, N_{3} \rightarrow \infty}} \int_{\epsilon_{1} \leq\left|u_{1}\right| \leq 4} \int_{\epsilon_{2} \leq\left|x_{2}-u_{2}\right| \leq N_{2}} \int_{\epsilon_{3} \leq\left|x_{3}-u_{3}\right| \leq N_{3}} \mathcal{K}\left(u_{1}, x_{2}-u_{2}, x_{3}-u_{3}\right) \\
& \times \phi^{(1)}\left(x_{1}\right) \phi^{(2)}\left(u_{2}, u_{3}\right) \mathrm{d} u_{3} \mathrm{~d} u_{2} \mathrm{~d} u_{1} .
\end{aligned}
$$

By the condition (RR) with $\alpha=\beta=\gamma=0$ and the smoothness condition of $\phi^{(1)}$ on the first integral and the condition (C2.b) with $\beta=\gamma=0$ for the second integral and the dominated convergent theorem, we have

$$
\begin{aligned}
|S| \lesssim & \int_{\left|u_{1}\right| \leq 4} \int_{\left|u_{2}\right| \leq 1} \int_{\left|u_{3}\right| \leq 1} \frac{1}{\left|u_{1}\right|\left|x_{2}\right|\left|x_{3}-u_{3}\right|}\left(\left|\frac{u_{1} x_{2}}{x_{3}-u_{3}}\right|+\left|\frac{x_{3}-u_{3}}{u_{1} x_{2}}\right|\right)^{-\lambda}\left|u_{1}\right| \mathrm{d} u_{3} \mathrm{~d} u_{2} \mathrm{~d} u_{1} \\
& +\int_{\left|u_{2}\right| \leq 1} \int_{\left|u_{3}\right| \leq 1} \frac{1}{\left|x_{2}\right|\left|x_{3}-u_{3}\right|}\left(\left|\frac{4 x_{2}}{x_{3}-u_{3}}\right|+\left|\frac{x_{3}-u_{3}}{4 x_{2}}\right|\right)^{-\lambda} \mathrm{d} u_{3} \mathrm{~d} u_{2} \\
& \lesssim \frac{1}{\left|x_{2}\right|^{1+\lambda}} \\
& \lesssim \frac{1}{(1+|x|)^{1+\lambda}\left(1+\left|x_{2}\right|\right)^{1+\lambda}\left(1+\left|x_{3}\right|\right)^{1+\lambda}} .
\end{aligned}
$$

Case $7\left|x_{1}\right|<3,\left|x_{2}\right|<3,\left|x_{3}\right| \geq 3$. The required estimate follows directly from the condition (RR):

$$
\begin{aligned}
|S| & \lesssim \int_{\left|u_{1}\right| \leq 1} \int_{\left|u_{2}\right| \leq 1} \int_{\left|u_{3}\right| \leq 1} \frac{1}{\left|x_{1}-u_{1}\right|\left|x_{2}-u_{2}\right|\left|x_{3}\right|}\left|\frac{x_{3}}{\left(x_{1}-u_{1}\right)\left(x_{2}-u_{2}\right)}\right|^{-\lambda} \mathrm{d} u_{3} \mathrm{~d} u_{2} \mathrm{~d} u_{1} \\
& \lesssim \frac{1}{\left|x_{3}\right|^{1+\lambda}} \\
& \lesssim \frac{1}{\left(1+\left|x_{1}\right|\right)^{1+\lambda}\left(1+\left|x_{2}\right|\right)^{1+\lambda}\left(1+\left|x_{3}\right|\right)^{1+\lambda}}
\end{aligned}
$$


Case $8\left|x_{1}\right|<3,\left|x_{2}\right|<3,\left|x_{3}\right|<3$. Inserting

$$
\begin{aligned}
\phi^{(1)} & \left(x_{1}-u_{1}\right) \phi^{(2)}\left(x_{2}-u_{2}, x_{3}-u_{3}\right) \\
= & {\left[\phi^{(1)}\left(x_{1}-u_{1}\right)-\phi^{(1)}\left(x_{1}\right)\right]\left[\phi^{(2)}\left(x_{2}-u_{2}, x_{3}-u_{3}\right)-\phi^{(2)}\left(x_{2}, x_{3}\right)\right] } \\
& +\phi^{(1)}\left(x_{1}\right)\left[\phi^{(2)}\left(x_{2}-u_{2}, x_{3}-u_{3}\right)-\phi^{(2)}\left(x_{2}, x_{3}\right)\right] \\
& +\left[\phi^{(1)}\left(x_{1}-u_{1}\right)-\phi^{(1)}\left(x_{1}\right)\right] \phi^{(2)}\left(x_{2}, x_{3}\right)+\phi^{(1)}\left(x_{1}\right) \phi^{(2)}\left(x_{2}, x_{3}\right)
\end{aligned}
$$

we write

$$
\begin{aligned}
S= & \lim _{\substack{\epsilon_{1}, \epsilon_{2}, \epsilon_{3} \rightarrow 0 \\
N_{1}, N_{2}, N_{3} \rightarrow \infty}} \int_{\epsilon_{1} \leq\left|u_{1}\right| \leq 4} \int_{\epsilon_{2} \leq\left|u_{2}\right| \leq 4} \int_{\epsilon_{3} \leq\left|u_{3}\right| \leq 4} \mathcal{K}\left(u_{1}, u_{2}, u_{3}\right) \\
& \times\left\{\left[\phi^{(1)}\left(x_{1}-u_{1}\right)-\phi^{(1)}\left(x_{1}\right)\right]\left[\phi^{(2)}\left(x_{2}-u_{2}, x_{3}-u_{3}\right)-\phi^{(2)}\left(x_{2}, x_{3}\right)\right]\right. \\
& +\phi^{(1)}\left(x_{1}\right)\left[\phi^{(2)}\left(x_{2}-u_{2}, x_{3}-u_{3}\right)-\phi^{(2)}\left(x_{2}, x_{3}\right)\right] \\
& +\left[\phi^{(1)}\left(x_{1}-u_{1}\right)-\phi^{(1)}\left(x_{1}\right)\right] \phi^{(2)}\left(x_{2}, x_{3}\right) \\
& \left.+\phi^{(1)}\left(x_{1}\right) \phi^{(2)}\left(x_{2}, x_{3}\right)\right\} \mathrm{d} u_{3} \mathrm{~d} u_{2} \mathrm{~d} u_{1}
\end{aligned}
$$

as four integrals. Using the condition (RR) with $\alpha=\beta=\gamma=0$ and the smoothness condition of $\phi^{(1)}$ for the first integral, the cancellation conditions (C2.b), (C2.c), and (C2.a) for the last three integrals, and the dominated convergent theorem, we obtain

$$
\begin{aligned}
|S| \lesssim & \int_{\left|u_{1}\right| \leq 4} \int_{\left|u_{2}\right| \leq 4} \int_{\left|u_{3}\right| \leq 4} \frac{1}{\left|u_{1}\right|\left|u_{2}\right|\left|u_{3}\right|}\left(\left|\frac{u_{1} u_{2}}{u_{3}}\right|+\left|\frac{u_{3}}{u_{1} u_{2}}\right|\right)^{-\theta_{2}} \\
& +\int_{\left|u_{2}\right| \leq 4} \int_{\left|u_{3}\right| \leq 4} \frac{1}{\left|u_{2}\right|\left|u_{3}\right|}\left(\left|\frac{4 u_{2}}{u_{3}}\right|+\left|\frac{u_{3}}{4 u_{2}}\right|\right)^{-\theta_{2}}\left(\left|u_{2}\right|+\left|u_{3}\right|\right) \mathrm{d} u_{3} \mathrm{~d} u_{2} \\
& \quad+\int_{\left|u_{1}\right| \leq 4} \frac{1}{\left|u_{1}\right|}\left|u_{1}\right| \mathrm{d} u_{1}+1 \\
\lesssim & 1 \\
\lesssim & \frac{1}{\left(1+\left|x_{1}\right|\right)^{1+\lambda}\left(1+\left|x_{2}\right|\right)^{1+\lambda}\left(1+\left|x_{3}\right|\right)^{1+\lambda}} .
\end{aligned}
$$

The proof of Lemma 3.2 is complete.

Recall that $\phi_{j, k}\left(u_{1}, u_{2}, u_{3}\right)=2^{-2(j+k)} \phi^{(1)}\left(2^{-j} u_{1}\right) \phi^{(2)}\left(2^{-k} u_{2}, 2^{-(j+k)} u_{3}\right)$, and the assumptions on $\mathcal{K}$ are invariant with respect to Zygmund dilation. By Lemma 3.2, we have the following estimate.

$$
\left|\left(\mathcal{K} * \phi_{j, k}\right)\left(x_{1}, x_{2}, x_{3}\right)\right| \leq C \frac{2^{-j}}{\left(1+2^{-j}\left|x_{1}\right|\right)^{1+\lambda}} \frac{2^{-k}}{\left(1+2^{-k}\left|x_{2}\right|\right)^{1+\lambda}} \frac{2^{-(j+k)}}{\left(1+2^{-(j+k)}\left|x_{3}\right|\right)^{1+\lambda}} .
$$

Now the proof of Proposition 3.1 follows from the above estimate with replacing $\phi_{j, k}$ by $\phi_{j, k} * \phi_{j^{\prime}, k^{\prime}}$. Note that $\phi_{j, k} * \phi_{j^{\prime}, k^{\prime}}$ satisfies the same properties as $\phi_{j \vee j^{\prime}, k \vee k^{\prime}}$ but 
with the bound $C 2^{-\left|j-j^{\prime}\right|} 2^{-\left|k-k^{\prime}\right|}$. Thus, the proof of Proposition 3.1 is complete and hence Theorem 1.3 is proved.

We now turn to the proof of Theorem 1.4. To prove part (a), we first show the $L^{2}$ boundedness of $\mathcal{K} * f$. This is similar to the proof of Theorem 1.1. We only outline the proof as follows:

By the Plancherel theorem, the $L^{2}$ boundedness of $\mathcal{K} * f$ is equivalent to $|\widehat{\mathcal{K}}(\chi, \eta, \xi)|$ $\leq A$, where $\widehat{\mathcal{K}}$ is the Fourier transform of $\mathcal{K}$ in the sense of distributions and $A$ is the constant depending only on the constant $C$.

Let $\zeta_{1}\left(x_{1}\right)$ be a smooth function on $\mathbb{R}$ with $\zeta_{1}\left(x_{1}\right)=1$ if $\left|x_{1}\right| \leq 8$ and $\zeta_{1}\left(x_{1}\right)=$ 0 if $\left|x_{1}\right| \geq 16$, and $\zeta_{2}=1-\zeta_{1}$. For simplicity, we denote by $\widetilde{\mathcal{K}}\left(x_{1}, x_{2}, x_{3}\right)=$ $\frac{1}{\chi \eta \xi} \mathcal{K}\left(\frac{x_{1}}{\chi}, \frac{x_{2}}{\eta}, \frac{x_{3}}{\xi}\right)$. We write

$$
\begin{aligned}
\widehat{\mathcal{K}}(\chi, \eta, \xi)= & \iiint \widetilde{\mathcal{K}}\left(x_{1}, x_{2}, x_{3}\right) \zeta_{2}\left(x_{1}\right) e^{-i x_{1}} e^{-i x_{2}} e^{-i x_{3}} \mathrm{~d} x_{1} \mathrm{~d} x_{2} \mathrm{~d} x_{3} \\
& +\iiint \widetilde{\mathcal{K}}\left(x_{1}, x_{2}, x_{3}\right) \zeta_{1}\left(x_{1}\right) e^{-i x_{1}} e^{-i x_{2}} e^{-i x_{3}} \mathrm{~d} x_{1} \mathrm{~d} x_{2} \mathrm{~d} x_{3} \\
:= & I+I I
\end{aligned}
$$

To estimate $I$, we write

$$
\begin{aligned}
|I|= & \frac{1}{2}\left|\iiint \Delta_{x_{1}, \pi}\left(\widetilde{\mathcal{K}}\left(x_{1}, x_{2}, x_{3}\right) \zeta_{2}\left(x_{1}\right)\right) e^{-i x_{1}} e^{-i x_{2}} e^{-i x_{3}} \mathrm{~d} x_{1} \mathrm{~d} x_{2} \mathrm{~d} x_{3}\right| \\
\lesssim & \left|\iiint \Delta_{x_{1}, \pi}\left(\widetilde{\mathcal{K}}\left(x_{1}, x_{2}, x_{3}\right) \zeta_{2}\left(x_{1}\right)\right) e^{-i x_{1}} \zeta_{2}\left(x_{2}\right) e^{-i x_{2}} e^{-i x_{3}} \mathrm{~d} x_{1} \mathrm{~d} x_{2} \mathrm{~d} x_{3}\right| \\
& +\left|\iiint \Delta_{x_{1}, \pi}\left(\widetilde{\mathcal{K}}\left(x_{1}, x_{2}, x_{3}\right) \zeta_{2}\left(x_{1}\right)\right) e^{-i x_{1}} \zeta_{1}\left(x_{2}\right) e^{-i x_{2}} e^{-i x_{3}} \mathrm{~d} x_{1} \mathrm{~d} x_{2} \mathrm{~d} x_{3}\right| \\
:= & I_{1}+I_{2} .
\end{aligned}
$$

Note that

$$
\begin{aligned}
I_{1} & \lesssim\left|\iiint \Delta_{x_{2}, \pi}\left(\Delta_{x_{1}, \pi}\left(\widetilde{\mathcal{K}}\left(x_{1}, x_{2}, x_{3}\right) \zeta_{2}\left(x_{1}\right)\right) \zeta_{2}\left(x_{2}\right)\right) e^{-i x_{1}} e^{-i x_{2}} e^{-i x_{3}} \mathrm{~d} x_{1} \mathrm{~d} x_{2} \mathrm{~d} x_{3}\right| \\
& \lesssim \int_{\mathbb{R}} \int_{\left|x_{2}\right| \geq 8} \int_{\left|x_{1}\right| \geq 8} \frac{1}{\left|x_{1}\right|^{1+\theta_{1}}\left|x_{2}\right|^{1+\theta_{1}}\left|x_{3}\right|}\left(\left|\frac{x_{1} x_{2} \xi}{x_{3} \chi \eta}\right|+\left|\frac{x_{3} \chi \eta}{x_{1} x_{2} \xi}\right|\right)^{-\theta_{2}} \mathrm{~d} x_{1} \mathrm{~d} x_{2} \mathrm{~d} x_{3} \lesssim 1 .
\end{aligned}
$$

For term $I_{2}$, note that

$$
\begin{aligned}
\left|I_{2}\right| \leq & \left|\iiint \Delta_{x_{1}, \pi}\left(\widetilde{\mathcal{K}}\left(x_{1}, x_{2}, x_{3}\right) \zeta_{2}\left(x_{1}\right)\right) e^{-i x_{1}} \zeta_{1}\left(x_{2}\right) e^{-i x_{2}} \zeta_{2}\left(x_{3}\right) e^{-i x_{3}} \mathrm{~d} x_{1} \mathrm{~d} x_{2} \mathrm{~d} x_{3}\right| \\
& +\left|\iiint \Delta_{x_{1}, \pi}\left(\widetilde{\mathcal{K}}\left(x_{1}, x_{2}, x_{3}\right) \zeta_{2}\left(x_{1}\right)\right) e^{-i x_{1}} \zeta_{1}\left(x_{2}\right) e^{-i x_{2}} \zeta_{1}\left(x_{3}\right) e^{-i x_{3}} \mathrm{~d} x_{1} \mathrm{~d} x_{2} \mathrm{~d} x_{3}\right| \\
& :=I_{2,1}+I_{2,2} .
\end{aligned}
$$


and

$$
\begin{aligned}
I_{2,1} & =\frac{1}{2}\left|\iiint \Delta_{x_{3}, \pi}\left(\Delta_{x_{1}, \pi}\left(\widetilde{\mathcal{K}}\left(x_{1}, x_{2}, x_{3}\right) \zeta_{2}\left(x_{1}\right)\right) \zeta_{2}\left(x_{3}\right)\right) e^{-i x_{1}} \zeta_{1}\left(x_{2}\right) e^{-i x_{2}} e^{-i x_{3}} \mathrm{~d} x_{1} \mathrm{~d} x_{2} \mathrm{~d} x_{3}\right| \\
& \lesssim \int_{\left|x_{3}\right| \geq 8} \int_{\left|x_{2}\right|<16} \int_{\left|x_{1}\right| \geq 8} \frac{1}{\left|x_{1}\right|^{1+\theta_{1}}\left|x_{2}\right|\left|x_{3}\right|^{1+\theta_{1}}}\left(\left|\frac{x_{1} x_{2} \xi}{x_{3} \chi \eta}\right|+\left|\frac{x_{3} \chi \eta}{x_{1} x_{2} \xi}\right|\right)^{-\theta_{2}} \mathrm{~d} x_{1} \mathrm{~d} x_{2} \mathrm{~d} x_{3} \\
& \lesssim 1
\end{aligned}
$$

To estimate $I_{2,2}$, we write

$$
\begin{aligned}
I_{2,2}= & \iiint \Delta_{x_{1}, \pi}\left(\widetilde{\mathcal{K}}\left(x_{1}, x_{2}, x_{3}\right) \zeta_{2}\left(x_{1}\right)\right) e^{-i x_{1}} \zeta_{1}\left(x_{2}\right) \zeta_{1}\left(x_{3}\right)\left(e^{-i x_{2}} e^{-i x_{3}}-1\right) \mathrm{d} x_{1} \mathrm{~d} x_{2} \mathrm{~d} x_{3} \\
& +\iiint \Delta_{x_{1}, \pi}\left(\widetilde{\mathcal{K}}\left(x_{1}, x_{2}, x_{3}\right) \zeta_{2}\left(x_{1}\right)\right) e^{-i x_{1}} \zeta_{1}\left(x_{2}\right) \zeta_{1}\left(x_{3}\right) \mathrm{d} x_{1} \mathrm{~d} x_{2} \mathrm{~d} x_{3} .
\end{aligned}
$$

Inserting $\left|e^{-i x_{2}} e^{-i x_{3}}-1\right| \leq\left|x_{2}\right|+\left|x_{3}\right|$ into the first integral together with the condition (RR) and using the cancellation condition (C3.c) for the second integral, respectively, we get

$$
\begin{aligned}
I_{2,2} \lesssim & \int_{\left|x_{3}\right|<16} \int_{\left|x_{2}\right|<16} \int_{\left|x_{1}\right| \geq 8} \frac{1}{\left|x_{1}\right|^{1+\theta_{1}\left|x_{2}\right|\left|x_{3}\right|}}\left(\left|\frac{x_{1} x_{2} \xi}{x_{3} \chi \eta}\right|+\left|\frac{x_{3} \chi \eta}{x_{1} x_{2} \xi}\right|\right)^{-\theta_{2}} \\
& \left(\left|x_{2}\right|+\left|x_{3}\right|\right) \mathrm{d} x_{2} \mathrm{~d} x_{3} \\
& +\int_{\left|x_{1}\right| \geq 8}\left|x_{1}\right|^{-1-\theta_{1}} \mathrm{~d} x_{1}
\end{aligned}
$$

which is dominated by the constant. Altogether, we obtain the required bound for term I.

Now we estimate term $I I$. We first write

$$
\begin{aligned}
I I= & \iiint \tilde{\mathcal{K}}\left(x_{1}, x_{2}, x_{3}\right) \zeta_{1}\left(x_{1}\right)\left(e^{-i x_{1}}-1\right) e^{-i x_{2}} e^{-i x_{3}} \mathrm{~d} x_{1} \mathrm{~d} x_{2} \mathrm{~d} x_{3} \\
& +\iiint \widetilde{\mathcal{K}}\left(x_{1}, x_{2}, x_{3}\right) \zeta_{1}\left(x_{1}\right) e^{-i x_{2}} e^{-i x_{3}} \mathrm{~d} x_{1} \mathrm{~d} x_{2} \mathrm{~d} x_{3} \\
& :=I I_{1}+I I_{2} .
\end{aligned}
$$

We further write

$$
\begin{aligned}
I I_{1}= & \iiint \tilde{\mathcal{K}}\left(x_{1}, x_{2}, x_{3}\right) \zeta_{1}\left(x_{1}\right)\left(e^{-i x_{1}}-1\right) \zeta_{2}\left(x_{2}\right) e^{-i x_{2}} e^{-i x_{3}} \mathrm{~d} x_{1} \mathrm{~d} x_{2} \mathrm{~d} x_{3} \\
& +\iiint \tilde{\mathcal{K}}\left(x_{1}, x_{2}, x_{3}\right) \zeta_{1}\left(x_{1}\right)\left(e^{-i x_{1}}-1\right) \zeta_{1}\left(x_{2}\right) e^{-i x_{2}} e^{-i x_{3}} \mathrm{~d} x_{1} \mathrm{~d} x_{2} \mathrm{~d} x_{3} \\
& :=I I_{1,1}+I I_{1,2} .
\end{aligned}
$$


For term $I I_{1,1}$, we have

$$
\left|I I_{1,1}\right|=\frac{1}{2}\left|\iiint \Delta_{x_{2}, \pi}\left(\widetilde{\mathcal{K}}\left(x_{1}, x_{2}, x_{3}\right) \zeta_{2}\left(x_{2}\right)\right) \zeta_{1}\left(x_{1}\right)\left(e^{-i x_{1}}-1\right) e^{-i x_{2}} e^{-i x_{3}} \mathrm{~d} x_{1} \mathrm{~d} x_{2} \mathrm{~d} x_{3}\right|
$$

Then the required bound follows from the fact that $\left|e^{-i x_{1}}-1\right| \leq\left|x_{1}\right|$ and the condition (RR).

Similarly, we write

$$
\begin{aligned}
I I_{1,2}= & \iiint \tilde{\mathcal{K}}\left(x_{1}, x_{2}, x_{3}\right) \zeta_{1}\left(x_{1}\right)\left(e^{-i x_{1}}-1\right) \zeta_{1}\left(x_{2}\right) e^{-i x_{2}} \zeta_{2}\left(x_{3}\right) e^{-i x_{3}} \mathrm{~d} x_{1} \mathrm{~d} x_{2} \mathrm{~d} x_{3} \\
& +\iiint \tilde{\mathcal{K}}\left(x_{1}, x_{2}, x_{3}\right) \zeta_{1}\left(x_{1}\right)\left(e^{-i x_{1}}-1\right) \zeta_{1}\left(x_{2}\right) e^{-i x_{2}} \zeta_{1}\left(x_{3}\right) e^{-i x_{3}} \mathrm{~d} x_{1} \mathrm{~d} x_{2} \mathrm{~d} x_{3} \\
& :=I I_{1,2,1}+I I_{1,2,2} .
\end{aligned}
$$

Since

$$
\begin{aligned}
& \left|I I_{1,2,1}\right| \\
& =\frac{1}{2}\left|\iiint \Delta_{x_{3}, \pi}\left(\widetilde{\mathcal{K}}\left(x_{1}, x_{2}, x_{3}\right) \zeta_{2}\left(x_{3}\right)\right) \zeta_{1}\left(x_{1}\right)\left(e^{-i x_{1}}-1\right) \zeta_{1}\left(x_{2}\right) e^{-i x_{2}} e^{-i x_{3}} \mathrm{~d} x_{1} \mathrm{~d} x_{2} \mathrm{~d} x_{3}\right| .
\end{aligned}
$$

The required bound for $I I_{1,2,1}$ then is concluded by the fact that $\left|e^{-i x_{1}}-1\right| \leq\left|x_{1}\right|$ and the condition (RR). To estimate term $I I_{1,2,2}$, we write

$$
\begin{aligned}
I I_{1,2,2}= & \iiint \widetilde{\mathcal{K}}\left(x_{1}, x_{2}, x_{3}\right) \zeta_{1}\left(x_{1}\right)\left(e^{-i x_{1}}-1\right) \zeta_{1}\left(x_{2}\right) \zeta_{1}\left(x_{3}\right)\left(e^{-i x_{2}} e^{-i x_{3}}-1\right) \mathrm{d} x_{1} \mathrm{~d} x_{2} \mathrm{~d} x_{3} \\
& +\iiint \widetilde{\mathcal{K}}\left(x_{1}, x_{2}, x_{3}\right) \zeta_{1}\left(x_{1}\right)\left(e^{-i x_{1}}-1\right) \zeta_{1}\left(x_{2}\right) \zeta_{1}\left(x_{3}\right) \mathrm{d} x_{1} \mathrm{~d} x_{2} \mathrm{~d} x_{3} .
\end{aligned}
$$

Using the facts that $\left|e^{-i x_{1}}-1\right| \leq\left|x_{1}\right|$ and $\left|e^{-i x_{2}} e^{-i x_{3}}-1\right| \leq\left|x_{2}\right|+\left|x_{3}\right|$ and the condition (RR) for the first integral and the condition (C3.c) for the second integral yields the desired bound for $I I_{1,2,2}$.

Finally, we estimate term $I I_{2}$. Denote $I I_{2}=I I_{2,1}+I I_{2,2}$, where $I I_{2,1}$ and $I I_{2,2}$ are given by $\iiint \widetilde{\mathcal{K}}\left(x_{1}, x_{2}, x_{3}\right) \zeta_{1}\left(x_{1}\right) \zeta_{2}\left(x_{2}\right) e^{-i x_{2}} e^{-i x_{3}} \mathrm{~d} x_{1} \mathrm{~d} x_{2} \mathrm{~d} x_{3}$ and $\iiint \widetilde{\mathcal{K}}\left(x_{1}, x_{2}, x_{3}\right) \zeta_{1}\left(x_{1}\right) \zeta_{1}\left(x_{2}\right) e^{-i x_{2}} e^{-i x_{3}} \mathrm{~d} x_{1} \mathrm{~d} x_{2} \mathrm{~d} x_{3}$, respectively. Then

$$
\left|I I_{2,1}\right|=\frac{1}{2}\left|\iiint \Delta_{x_{2}, \pi}\left(\widetilde{\mathcal{K}}\left(x_{1}, x_{2}, x_{3}\right) \zeta_{2}\left(x_{2}\right)\right) \zeta_{1}\left(x_{1}\right) e^{-i x_{2}} e^{-i x_{3}} \mathrm{~d} x_{1} \mathrm{~d} x_{2} \mathrm{~d} x_{3}\right| \lesssim 1
$$

For $I I_{2,2}$, inserting

$$
\begin{aligned}
\zeta_{1}\left(x_{1}\right) \zeta_{1}\left(x_{2}\right) e^{-i x_{2}} e^{-i x_{3}}= & \zeta_{1}\left(x_{1}\right) \zeta_{1}\left(x_{2}\right) e^{-i x_{2}} \zeta_{2}\left(x_{3}\right) e^{-i x_{3}} \\
& +\zeta_{1}\left(x_{1}\right) \zeta_{1}\left(x_{2}\right) \zeta_{1}\left(x_{3}\right)\left(e^{-i x_{2}} e^{-i x_{3}}-1\right)+\zeta_{1}\left(x_{1}\right) \zeta_{1}\left(x_{2}\right) \zeta_{1}\left(x_{3}\right)
\end{aligned}
$$


into

$$
\iiint \tilde{\mathcal{K}}\left(x_{1}, x_{2}, x_{3}\right) \zeta_{1}\left(x_{1}\right) \zeta_{1}\left(x_{2}\right) e^{-i x_{2}} e^{-i x_{3}} \mathrm{~d} x_{1} \mathrm{~d} x_{2} \mathrm{~d} x_{3}
$$

and using the condition (C1.b) and (C1.a), respectively. Thus, these estimates yield the bound of $I I_{2,2}$ and hence the required bound for term $I I$. The $L^{2}$ boundedness of $\mathcal{K} * f$ follows.

Next, to show the $L^{p}$ boundedness of the operator $\mathcal{K} * f$, similar to the proof of Theorem 1.3, it suffices to prove the following lemma.

Lemma 3.3 Suppose that $\phi^{(1)}$ and $\phi^{(2)}$ satisfy the conditions (3.1)-(3.4) and $\mathcal{K}$ is a distribution defined on $\mathbb{R}^{3}$ and satisfies the conditions (RR) and (C3.a)-(C3.c). Then for $\lambda=\frac{1}{2} \min \left(\theta_{1}, \theta_{2}\right)$,

$$
\left|\mathcal{K} *\left(\phi^{(1)} \otimes \phi^{(2)}\right)\left(x_{1}, x_{2}, x_{3}\right)\right| \leq \frac{C_{\lambda}}{\left(1+\left|x_{1}\right|\right)^{1+\lambda}\left(1+\left|x_{2}\right|\right)^{1+\lambda}\left(1+\left|x_{3}\right|\right)^{1+\lambda}}
$$

where $C_{\lambda}$ is the constant depending only on $\lambda$.

Proof The proof the Lemma 3.3 is similar to the proof of lemma 3.2. For simplicity, let $S=\mathcal{K} *\left(\phi^{(1)} \otimes \phi^{(2)}\right)\left(x_{1}, x_{2}, x_{3}\right)$. We consider the following eight cases:

Case $1\left|x_{1}\right| \geq 3,\left|x_{2}\right| \geq 3,\left|x_{3}\right| \geq 3$. For this case, we use (3.4) to write

$$
\begin{aligned}
S= & \iiint\left[\mathcal{K}\left(x_{1}-u_{1}, x_{2}-u_{2}, x_{3}-u_{3}\right)-\mathcal{K}\left(x_{1}, x_{2}-u_{2}, x_{3}-u_{3}\right)\right. \\
& \left.-\mathcal{K}\left(x_{1}-u_{1}, x_{2}, x_{3}\right)+\mathcal{K}\left(x_{1}, x_{2}, x_{3}\right)\right] \phi^{(1)}\left(u_{1}\right) \phi^{(2)}\left(u_{2}, u_{3}\right) \mathrm{d} u_{3} \mathrm{~d} u_{2} \mathrm{~d} u_{1} .
\end{aligned}
$$

Note that $\mathcal{K}\left(x_{1}-u_{1}, x_{2}-u_{2}, x_{3}-u_{3}\right)-\mathcal{K}\left(x_{1}, x_{2}-u_{2}, x_{3}-u_{3}\right)-\left(\mathcal{K}\left(x_{1}-u_{1}, x_{2}, x_{3}\right)-\right.$ $\left.\mathcal{K}\left(x_{1}, x_{2}, x_{3}\right)\right)=\Delta_{x_{2},-u_{2}} \Delta_{x_{1},-u_{1}} \mathcal{K}\left(x_{1}, x_{2}, x_{3}-u_{3}\right)+\Delta_{x_{3},-u_{3}} \Delta_{x_{1},-u_{1}} \mathcal{K}\left(x_{1}, x_{2}, x_{3}\right)$. Thus, by the condition (RR) with $\alpha=\beta=1, \gamma=0$ and $\alpha=\gamma=1, \beta=0$ respectively, we have

$$
\begin{aligned}
|S| \lesssim & \int_{\left|u_{1}\right| \leq 1} \int_{\left|u_{2}\right| \leq 1} \int_{\left|u_{3}\right| \leq 1} \frac{\left|u_{1}\right|^{\theta_{1}}\left|u_{2}\right|^{\theta_{1}}}{\left|x_{1}\right|^{1+\theta_{1}}\left|x_{2}\right|^{1+\theta_{1}\left|x_{3}\right|}}\left(\left|\frac{x_{1} x_{2}}{x_{3}}\right|+\left|\frac{x_{3}}{x_{1} x_{2}}\right|\right)^{-\lambda} \mathrm{d} u_{3} \mathrm{~d} u_{2} \mathrm{~d} u_{1} \\
& +\int_{\left|u_{1}\right| \leq 1} \int_{\left|u_{2}\right| \leq 1} \int_{\left|u_{3}\right| \leq 1} \frac{\left|u_{1}\right|^{\theta_{1}}\left|u_{3}\right|^{\theta_{1}}}{\left|x_{1}\right|^{1+\theta_{1}}\left|x_{2}\right|\left|x_{3}\right|^{1+\theta_{1}}}\left(\left|\frac{x_{1} x_{2}}{x_{3}}\right|+\left|\frac{x_{3}}{x_{1} x_{2}}\right|\right)^{-\lambda} \mathrm{d} u_{3} \mathrm{~d} u_{2} \mathrm{~d} u_{1} \\
& \lesssim \frac{1}{\left(1+\left|x_{1}\right|\right)^{1+\lambda}\left(1+\left|x_{2}\right|\right)^{1+\lambda}\left(1+\left|x_{3}\right|\right)^{1+\lambda}} .
\end{aligned}
$$

Case $2\left|x_{1}\right| \geq 3,\left|x_{2}\right| \geq 3,\left|x_{3}\right|<3$. By the cancellation condition of $\phi^{(1)}$,

$$
\begin{aligned}
S= & \iiint\left[\mathcal{K}\left(x_{1}-u_{1}, x_{2}-u_{2}, x_{3}-u_{3}\right)-\mathcal{K}\left(x_{1}, x_{2}-u_{2}, x_{3}-u_{3}\right)\right] \\
& \times \phi^{(1)}\left(u_{1}\right) \phi^{(2)}\left(u_{2}, u_{3}\right) \mathrm{d} u_{3} \mathrm{~d} u_{2} \mathrm{~d} u_{1} .
\end{aligned}
$$


Therefore, by the condition (RR) with $\alpha=1$ and $\beta=\gamma=0$, we obtain

$$
\begin{aligned}
|S| & \lesssim \int_{\left|u_{1}\right| \leq 1} \int_{\left|u_{2}\right| \leq 1} \int_{\left|u_{3}\right| \leq 1} \frac{\left|u_{1}\right|^{\theta_{1}}}{\left|x_{1}\right|^{1+\theta_{1}\left|x_{2}\right|\left|x_{3}-u_{3}\right|}\left|\frac{x_{1} x_{2}}{x_{3}-u_{3}}\right|^{-\lambda} \mathrm{d} u_{3} \mathrm{~d} u_{2} \mathrm{~d} u_{1}} \\
& \lesssim \frac{1}{\left(1+\left|x_{1}\right|\right)^{1+\lambda}\left(1+\left|x_{2}\right|\right)^{1+\lambda}\left(1+\left|x_{3}\right|\right)^{1+\lambda}}
\end{aligned}
$$

Case $3\left|x_{1}\right| \geq 3,\left|x_{2}\right|<3,\left|x_{3}\right| \geq 3$. The same expression for $S$ as in Case 2 yields

$$
\begin{aligned}
|S| & \lesssim \int_{\left|u_{1}\right| \leq 1} \int_{\left|u_{2}\right| \leq 1} \int_{\left|u_{3}\right| \leq 1} \frac{\left|u_{1}\right|^{\theta_{1}}}{\left|x_{1}\right|^{1+\theta_{1}\left|x_{2}-u_{2}\right|\left|x_{3}\right|}}\left|\frac{x_{3}}{x_{1}\left(x_{2}-u_{2}\right)}\right|^{-\lambda} \mathrm{d} u_{3} \mathrm{~d} u_{2} \mathrm{~d} u_{1} \\
& \lesssim \frac{1}{\left(1+\left|x_{1}\right|\right)^{1+\lambda}\left(1+\left|x_{2}\right|\right)^{1+\lambda}\left(1+\left|x_{3}\right|\right)^{1+\lambda}} .
\end{aligned}
$$

Before handling the other cases, we introduce a bump function $\widetilde{\phi}$ on $\mathbb{R}$, with $\widetilde{\phi}\left(x_{1}\right)=$ 1 if $\left|x_{1}\right| \leq 1 / 2$ and $\widetilde{\phi}\left(x_{1}\right)=0$ if $\left|x_{1}\right| \geq 1$.

Case $4\left|x_{1}\right| \geq 3,\left|x_{2}\right|<3,\left|x_{3}\right|<3$. Using the cancellation condition of $\phi^{(1)}$, we write

$$
\begin{array}{rl}
\mathcal{K} & *\left(\phi^{(1)} \otimes \phi^{(2)}\right)\left(x_{1}, x_{2}, x_{3}\right) \\
= & \iiint\left(\mathcal{K}\left(x_{1}-u_{1}, u_{2}, u_{3}\right)-\mathcal{K}\left(x, u_{2}, u_{3}\right)\right) \phi^{(1)}\left(u_{1}\right) \\
& \times\left(\phi^{(2)}\left(x_{2}-u_{2}, x_{3}-u_{3}\right)-\phi^{(2)}\left(x_{2}, x_{3}\right)\right) \widetilde{\phi}\left(\frac{u_{2}}{10}\right) \widetilde{\phi}\left(\frac{u_{3}}{10}\right) \mathrm{d} u_{3} \mathrm{~d} u_{2} \mathrm{~d} u_{1} \\
& +\iiint\left(\mathcal{K}\left(x_{1}-u_{1}, u_{2}, u_{3}\right)-\mathcal{K}\left(x_{1}, u_{2}, u_{3}\right)\right) \phi^{(1)}\left(u_{1}\right) \\
& \times \phi^{(2)}\left(x_{2}, x_{3}\right) \tilde{\phi}\left(\frac{u_{2}}{10}\right) \widetilde{\phi}\left(\frac{u_{3}}{10}\right) \mathrm{d} u_{3} \mathrm{~d} u_{2} \mathrm{~d} u_{1} .
\end{array}
$$

Hence, by the condition (RR) with $\alpha=1, \beta=\gamma=0$ for the first integral, and the cancellation condition (C3.c) with $\alpha=1$ for the second integral, we get

$$
\begin{aligned}
|S| \lesssim & \int_{\left|u_{1}\right| \leq 1} \int_{\left|u_{2}\right| \leq 10} \int_{\left|u_{3}\right| \leq 10} \frac{\left|u_{1}\right|^{\theta_{1}}}{\left|x_{1}\right|^{1+\theta_{1}|| u_{2}|| u_{3} \mid}}\left(\left|\frac{x_{1} u_{2}}{u_{3}}\right|+\left|\frac{u_{3}}{x_{1} u_{2}}\right|\right)^{-\lambda}\left(\left|u_{2}\right|+\left|u_{3}\right|\right) \mathrm{d} u_{3} \mathrm{~d} u_{2} \mathrm{~d} u_{1} \\
& +\int_{\left|u_{1}\right| \leq 1} \frac{\left|u_{1}\right|^{\theta_{1}}}{\left|x_{1}\right|^{1+\theta_{1}}} \mathrm{~d} u_{1} \\
\lesssim & \frac{1}{\left(1+\left|x_{1}\right|\right)^{1+\lambda}\left(1+\left|x_{2}\right|\right)^{1+\lambda}\left(1+\left|x_{3}\right|\right)^{1+\lambda}} .
\end{aligned}
$$

Case $5\left|x_{1}\right|<3,\left|x_{2}\right| \geq 3,\left|x_{3}\right| \geq 3$. Similar to Case 4, using the cancellation condition of $\phi^{(2)}$, we write

$$
\begin{aligned}
S= & \iiint\left[\mathcal{K}\left(u_{1}, x_{2}-u_{2}, x_{3}-u_{3}\right)-\mathcal{K}\left(u_{1}, x_{2}, x_{3}\right)\right] \\
& \times\left(\phi^{(1)}\left(x_{1}-u_{1}\right)-\phi^{(1)}\left(x_{1}\right)\right) \phi^{(2)}\left(u_{2}, u_{3}\right) \tilde{\phi}\left(\frac{u_{1}}{10}\right) \mathrm{d} u_{3} \mathrm{~d} u_{2} \mathrm{~d} u_{1}
\end{aligned}
$$




$$
\begin{aligned}
& +\iiint\left[\mathcal{K}\left(u_{1}, x_{2}-u_{2}, x_{3}-u_{3}\right)-\mathcal{K}\left(u_{1}, x_{2}, x_{3}\right)\right] \phi^{(1)}\left(x_{1}\right) \phi^{(2)}\left(u_{2}, u_{3}\right) \tilde{\phi} \\
& \quad\left(\frac{u_{1}}{10}\right) \mathrm{d} u_{3} \mathrm{~d} u_{2} \mathrm{~d} u_{1} .
\end{aligned}
$$

Note that $\mathcal{K}\left(u_{1}, x_{2}-u_{2}, x_{3}-u_{3}\right)-\mathcal{K}\left(u_{1}, x_{2}, x_{3}\right)=\Delta_{x_{2},-u_{2}} \mathcal{K}\left(u_{1}, x_{2}, x_{3}-u_{3}\right)+$ $\Delta_{x_{3},-u_{3}} \mathcal{K}\left(u_{1}, x_{2}, x_{3}\right)$. Thus, using the condition (RR) on $\mathcal{K}$, the smoothness of $\phi^{(1)}$ for the first integral, and the cancellation conditions (C3.b) with $\beta=1, \gamma=0$ and $\beta=0, \gamma=1$, respectively, for the second integral, and applying the dominated convergence theorem, we obtain

$$
\begin{aligned}
& |S| \lesssim \int_{\left|u_{1}\right| \leq 10} \int_{\left|u_{2}\right| \leq 1} \int_{\left|u_{3}\right| \leq 1}\left(\frac{\left|u_{2}\right|^{\theta_{1}}}{\left|u_{1}\right|\left|x_{2}\right|^{1+\theta_{1}}\left|x_{3}\right|}+\frac{\left|u_{3}\right|^{\theta_{1}}}{\left|u_{1}\right|\left|x_{2}\right|\left|x_{3}\right|^{1+\theta_{1}}}\right) \\
& \times\left(\left|\frac{u_{1} x_{2}}{x_{3}}\right|+\left|\frac{x_{3}}{u_{1} x_{2}}\right|\right)^{-\lambda}\left|u_{1}\right| \mathrm{d} u_{3} \mathrm{~d} u_{2} \mathrm{~d} u_{1} \\
& +\int_{\left|u_{2}\right| \leq 1} \int_{\left|u_{3}\right| \leq 1}\left(\frac{\left|u_{2}\right|^{\theta_{1}}}{\left|x_{2}\right|^{1+\theta_{1}}\left|x_{3}\right|}+\frac{\left|u_{3}\right|^{\theta_{1}}}{\left|x_{2}\right|\left|x_{3}\right|^{1+\theta_{1}}}\right)\left(\left|\frac{4 x_{2}}{x_{3}}\right|+\left|\frac{x_{3}}{4 x_{2}}\right|\right)^{-\lambda} \mathrm{d} u_{3} \mathrm{~d} u_{2} \\
& \lesssim \frac{1}{\left(1+\left|x_{1}\right|\right)^{1+\lambda}\left(1+\left|x_{2}\right|\right)^{1+\lambda}\left(1+\left|x_{3}\right|\right)^{1+\lambda}}
\end{aligned}
$$

Case $6\left|x_{1}\right|<3,\left|x_{2}\right| \geq 3,\left|x_{3}\right|<3$. Note that

$$
\begin{aligned}
S= & \iiint \mathcal{K}\left(u_{1}, x_{2}-u_{2}, x_{3}-u_{3}\right)\left(\phi^{(1)}\left(x_{1}-u_{1}\right)-\phi^{(1)}\left(x_{1}\right)\right) \phi^{(2)}\left(u_{2}, u_{3}\right) \tilde{\phi} \\
& \left(\frac{u_{1}}{10}\right) \mathrm{d} u_{3} \mathrm{~d} u_{2} \mathrm{~d} u_{1} \\
& +\iiint \mathcal{K}\left(u_{1}, x_{2}-u_{2}, x_{3}-u_{3}\right) \phi^{(1)}\left(x_{1}\right) \phi^{(2)}\left(u_{2}, u_{3}\right) \tilde{\phi}\left(\frac{u_{1}}{10}\right) \mathrm{d} u_{3} \mathrm{~d} u_{2} \mathrm{~d} u_{1} .
\end{aligned}
$$

By the condition (RR) with $\alpha=\beta=\gamma=0$ and the smoothness condition of $\phi^{(1)}$ on the first integral and the condition (C3.b) with $\beta=\gamma=0$ for the second integral, we have

$$
\begin{aligned}
|S| \lesssim & \int_{\left|u_{1}\right| \leq 10} \int_{\left|u_{2}\right| \leq 1} \int_{\left|u_{3}\right| \leq 1} \frac{1}{\left|u_{1}\right|\left|x_{2}\right|\left|x_{3}-u_{3}\right|}\left(\left|\frac{u_{1} x_{2}}{x_{3}-u_{3}}\right|+\left|\frac{x_{3}-u_{3}}{u_{1} x_{2}}\right|\right)^{-\lambda} \\
& +\int_{\left|u_{2}\right| \mathrm{d} u_{3} \mathrm{~d} u_{2} \mathrm{~d} u_{1}} \int_{\left|u_{3}\right| \leq 1} \frac{1}{\left|x_{2}\right|\left|x_{3}-u_{3}\right|}\left(\left|\frac{x_{2}}{x_{3}-u_{3}}\right|+\left|\frac{x_{3}-u_{3}}{x_{2}}\right|\right)^{-\lambda} \mathrm{d} u_{3} \mathrm{~d} u_{2} \\
\lesssim & \frac{1}{\left|x_{2}\right|^{1+\lambda}} \lesssim \frac{1}{\left(1+\left|x_{1}\right|\right)^{1+\lambda}\left(1+\left|x_{2}\right|\right)^{1+\lambda}\left(1+\left|x_{3}\right|\right)^{1+\lambda}} .
\end{aligned}
$$


Case $7\left|x_{1}\right|<3,\left|x_{2}\right|<3,\left|x_{3}\right| \geq 3$. The required estimate follows directly from the condition (RR):

$$
\begin{aligned}
|S| & \lesssim \int_{\left|u_{1}\right| \leq 1} \int_{\left|u_{2}\right| \leq 1} \int_{\left|u_{3}\right| \leq 1} \frac{1}{\left|x_{1}-u_{1}\right|\left|x_{2}-u_{2}\right|\left|x_{3}\right|}\left|\frac{x_{3}}{\left(x_{1}-u_{1}\right)\left(x_{2}-u_{2}\right)}\right|^{-\lambda} \mathrm{d} u_{3} \mathrm{~d} u_{2} \mathrm{~d} u_{1} \\
& \lesssim \frac{1}{\left|x_{3}\right|^{1+\lambda}} \lesssim \frac{1}{\left(1+\left|x_{1}\right|\right)^{1+\lambda}\left(1+\left|x_{2}\right|\right)^{1+\lambda}\left(1+\left|x_{3}\right|\right)^{1+\lambda}}
\end{aligned}
$$

Case $8\left|x_{1}\right|<3,\left|x_{2}\right|<3,\left|x_{3}\right|<3$. Inserting

$$
\begin{aligned}
\phi^{(1)}\left(x_{1}-u_{1}\right) \phi^{(2)}\left(x_{2}-u_{2}, x_{3}-u_{3}\right) \\
=\left[\phi^{(1)}\left(x_{1}-u_{1}\right)-\phi^{(1)}\left(x_{1}\right)\right]\left[\phi^{(2)}\left(x_{2}-u_{2}, x_{3}-u_{3}\right)-\phi^{(2)}\left(x_{2}, x_{3}\right)\right] \\
\quad+\phi^{(1)}\left(x_{1}\right)\left[\phi^{(2)}\left(x_{2}-u_{2}, x_{3}-u_{3}\right)-\phi^{(2)}\left(x_{2}, x_{3}\right)\right] \\
+\left[\phi^{(1)}\left(x_{1}-u_{1}\right)-\phi^{(1)}\left(x_{1}\right)\right] \phi^{(2)}\left(x_{2}, x_{3}\right)+\phi^{(1)}\left(x_{1}\right) \phi^{(2)}\left(x_{2}, x_{3}\right),
\end{aligned}
$$

we write

$$
\begin{aligned}
S= & \iiint \mathcal{K}\left(u_{1}, u_{2}, u_{3}\right) \times\left\{[ \phi ^ { ( 1 ) } ( x _ { 1 } - u _ { 1 } ) - \phi ^ { ( 1 ) } ( x _ { 1 } ) ] \left[\phi^{(2)}\left(x_{2}-u_{2}, x_{3}-u_{3}\right)\right.\right. \\
& \left.-\phi^{(2)}\left(x_{2}, x_{3}\right)\right] \\
+ & \phi^{(1)}\left(x_{1}\right)\left[\phi^{(2)}\left(x_{2}-u_{2}, x_{3}-u_{3}\right)-\phi^{(2)}\left(x_{2}, x_{3}\right)\right]+\left[\phi^{(1)}\left(x_{1}-u_{1}\right)\right. \\
& \left.-\phi^{(1)}\left(x_{1}\right)\right] \phi^{(2)}\left(x_{2}, x_{3}\right) \\
+ & \left.\phi^{(1)}\left(x_{1}\right) \phi^{(2)}\left(x_{2}, x_{3}\right)\right\} \widetilde{\phi}\left(\frac{u_{1}}{10}\right) \widetilde{\phi}\left(\frac{u_{2}}{10}\right) \widetilde{\phi}\left(\frac{u_{3}}{10}\right) \mathrm{d} u_{3} \mathrm{~d} u_{2} \mathrm{~d} u_{1}
\end{aligned}
$$

as four integrals. Using the condition (RR) with $\alpha=\beta=\gamma=0$ and the smoothness condition of $\phi^{(1)}$ for the first integral, the cancellation conditions (C3.b), (C3.c), and (C3.a) for the last three integrals, we obtain

$$
\begin{aligned}
|S| \lesssim & \int_{\left|u_{1}\right| \leq 4} \int_{\left|u_{2}\right| \leq 4} \int_{\left|u_{3}\right| \leq 4} \frac{1}{\left|u_{1}\right|\left|u_{2}\right|\left|u_{3}\right|}\left(\left|\frac{u_{1} u_{2}}{u_{3}}\right|+\left|\frac{u_{3}}{u_{1} u_{2}}\right|\right)^{-\theta_{2}} \\
& +\int_{\left|u_{1}\right|\left(\left|u_{2}\right|+\left|u_{3}\right|\right) \mathrm{d} u_{3} \mathrm{~d} u_{2} \mathrm{~d} u_{1}} \int_{\left|u_{3}\right| \leq 4} \frac{1}{\left|u_{2}\right|\left|u_{3}\right|}\left(\left|\frac{4 u_{2}}{u_{3}}\right|+\left|\frac{u_{3}}{4 u_{2}}\right|\right)^{-\theta_{2}}\left(\left|u_{2}\right|+\left|u_{3}\right|\right) \mathrm{d} u_{3} \mathrm{~d} u_{2} \\
& +\int_{\left|u_{1}\right| \leq 4} \frac{1}{\left|u_{1}\right|}\left|u_{1}\right| \mathrm{d} u_{1}+1 \\
& \lesssim 1 \lesssim \frac{1}{\left(1+\left|x_{1}\right|\right)^{1+\lambda}\left(1+\left|x_{2}\right|\right)^{1+\lambda}\left(1+\left|x_{3}\right|\right)^{1+\lambda}}
\end{aligned}
$$

This completes the proof of Lemma 3.3. 
The proof of part (b) of Theorem 1.4 follows from part (a). Indeed, the conditions (RR) and (C2.a)-(C2.c) imply the conditions (C3.a)-(C3.c). To see this, inserting

$$
\begin{aligned}
\widetilde{\phi}\left(x_{1}, x_{2}, x_{3}\right)= & {\left[\left(\widetilde{\phi}\left(x_{1}, x_{2}, x_{3}\right)-\widetilde{\phi}\left(0, x_{2}, x_{3}\right)\right)-\left(\widetilde{\phi}\left(x_{1}, 0,0\right)-\widetilde{\phi}(0,0,0)\right)\right] } \\
& +\left(\widetilde{\phi}\left(x_{1}, 0,0\right)-\widetilde{\phi}(0,0,0)\right)+\left(\widetilde{\phi}\left(0, x_{2}, x_{3}\right)-\widetilde{\phi}\left(0, x_{2}, 0\right)\right) \\
& +\left(\widetilde{\phi}\left(0, x_{2}, 0\right)-\widetilde{\phi}(0,0,0)\right)+\widetilde{\phi}(0,0,0)
\end{aligned}
$$

into $\iiint \mathcal{K}\left(x_{1}, x_{2}, x_{3}\right) \widetilde{\phi}\left(x_{1}, x_{2}, x_{3}\right) \mathrm{d} x_{1} \mathrm{~d} x_{2} \mathrm{~d} x_{3}$, we obtain that

$$
\begin{aligned}
& \iiint \mathcal{K}\left(x_{1}, x_{2}, x_{3}\right) \widetilde{\phi}\left(R_{1} x_{1}, R_{2} x_{2}, R_{1} R_{2} x_{3}\right) \mathrm{d} x_{1} \mathrm{~d} x_{2} \mathrm{~d} x_{3} \\
& \quad=\lim _{\epsilon_{1}, \epsilon_{2}, \epsilon_{3} \rightarrow 0} \iiint_{E\left(\epsilon, R_{1}, R_{2}\right)} \mathcal{K}\left(x_{1}, x_{2}, x_{3}\right) \widetilde{\phi}\left(R_{1} x_{1}, R_{2} x_{2}, R_{1} R_{2} x_{3}\right) \mathrm{d} x_{1} \mathrm{~d} x_{2} \mathrm{~d} x_{3} .
\end{aligned}
$$

Let $E\left(\epsilon, R_{1}, R_{2}\right)=\left\{x \in \mathbb{R}^{3}: \epsilon_{1} \leq\left|x_{1}\right| \leq \frac{1}{R_{1}}, \epsilon_{2} \leq\left|x_{2}\right| \leq \frac{1}{R_{2}}, \epsilon_{3} \leq\left|x_{3}\right| \leq \frac{1}{R_{1} R_{2}}\right\}$. Then we have

$$
\begin{aligned}
& \left|\iiint_{E\left(\epsilon, R_{1}, R_{2}\right)} \mathcal{K}\left(x_{1}, x_{2}, x_{3}\right) \tilde{\phi}\left(R_{1} x_{1}, R_{2} x_{2}, R_{1} R_{2} x_{3}\right) \mathrm{d} x_{1} \mathrm{~d} x_{2} \mathrm{~d} x_{3}\right| \\
& \leq \mid \iiint_{E\left(\epsilon, R_{1}, R_{2}\right)} \mathcal{K}\left(x_{1}, x_{2}, x_{3}\right)\left\{\left[\widetilde{\phi}\left(R_{1} x_{1}, R_{2} x_{2}, R_{1} R_{2} x_{3}\right)-\widetilde{\phi}\left(0, R_{2} x_{2}, R_{1} R_{2} x_{3}\right)\right]\right. \\
& \left.-\left[\widetilde{\phi}\left(R_{1} x_{1}, 0,0\right)-\widetilde{\phi}(0,0,0)\right]\right\} \mathrm{d} x_{1} \mathrm{~d} x_{2} \mathrm{~d} x_{3} \mid \\
& +\left|\iiint_{E\left(\epsilon, R_{1}, R_{2}\right)} \mathcal{K}\left(x_{1}, x_{2}, x_{3}\right)\left(\widetilde{\phi}\left(R_{1} x_{1}, 0,0\right)-\widetilde{\phi}(0,0,0)\right) \mathrm{d} x_{1} \mathrm{~d} x_{2} \mathrm{~d} x_{3}\right| \\
& +\left|\iiint_{E\left(\epsilon, R_{1}, R_{2}\right)} \mathcal{K}\left(x_{1}, x_{2}, x_{3}\right)\left(\widetilde{\phi}\left(0, R_{2} x_{2}, R_{1} R_{2} x_{3}\right)-\widetilde{\phi}\left(0, R_{2} x_{2}, 0\right)\right) \mathrm{d} x_{1} \mathrm{~d} x_{2} \mathrm{~d} x_{3}\right| \\
& +\left|\iiint_{E\left(\epsilon, R_{1}, R_{2}\right)} \mathcal{K}\left(x_{1}, x_{2}, x_{3}\right)\left(\widetilde{\phi}\left(0, R_{2} x_{2}, 0\right)-\widetilde{\phi}(0,0,0)\right) \mathrm{d} x_{1} \mathrm{~d} x_{2} \mathrm{~d} x_{3}\right| \\
& +\left|\iiint_{E\left(\epsilon, R_{1}, R_{2}\right)} \mathcal{K}\left(x_{1}, x_{2}, x_{3}\right) \widetilde{\phi}(0,0,0) \mathrm{d} x_{1} \mathrm{~d} x_{2} \mathrm{~d} x_{3}\right| \\
& \lesssim \int_{\left|x_{3}\right| \leq \frac{1}{R_{1} R_{2}}} \int_{\left|x_{2}\right| \leq \frac{1}{R_{2}}} \int_{\left|x_{1}\right| \leq \frac{1}{R_{1}}} \frac{1}{\left|x_{1}\right|\left|x_{2}\right|\left|x_{3}\right|}\left(\left|\frac{x_{1} x_{2}}{x_{3}}\right|+\left|\frac{x_{3}}{x_{1} x_{2}}\right|\right)^{-\theta_{2}} \\
& \times\left|R_{1} x_{1}\right|\left(\left|R_{2} x_{2}\right|+\left|R_{1} R_{2} x_{3}\right|\right) \mathrm{d} x_{1} \mathrm{~d} x_{2} \mathrm{~d} x_{3} \\
& +\int_{\left|x_{1}\right| \leq \frac{1}{R_{1}}} \frac{1}{\left|x_{1}\right|}\left|R_{1} x_{1}\right| \mathrm{d} x_{1} \\
& +\int_{\left|x_{3}\right| \leq \frac{1}{R_{1} R_{2}}} \int_{\mathbb{R}} \frac{1}{\left|x_{2}\right|\left|x_{3}\right|}\left(\left|\frac{x_{2}}{R_{1} x_{3}}\right|+\left|\frac{R_{1} x_{3}}{x_{2}}\right|\right)^{-\theta_{2}}\left|R_{1} R_{2} x_{3}\right| \mathrm{d} x_{2} \mathrm{~d} x_{3} \\
& +\left|\int_{\left|x_{2}\right| \leq \frac{1}{R_{2}}} \frac{1}{\left|x_{2}\right|}\right| R_{2} x_{2} \mid \mathrm{d} x_{2}+1 \lesssim 1
\end{aligned}
$$


where we apply (RR) and (C2.c) for the first and second term, respectively, (C2.b) for the third and fourth term, and (C2.a) for the last term above, which imply that $\mathcal{K}$ satisfies (C3.a).

Similarly, for any $0 \leq \beta+\gamma \leq 1$, n.b.f. $\widetilde{\phi}$ on $\mathbb{R}$ and $R>0$, we can write

$$
\begin{aligned}
& \left|\Delta_{x_{2}, h_{2}}^{\beta} \Delta_{x_{3}, h_{3}}^{\gamma} \mathcal{K}\left(x_{1}, x_{2}, x_{3}\right) \tilde{\phi}\left(R x_{1}\right) \mathrm{d} x_{1}\right| \\
& =\lim _{\epsilon \rightarrow 0}\left|\int_{\epsilon \leq\left|x_{1}\right| \leq \frac{1}{R}} \Delta_{x_{2}, h_{2}}^{\beta} \Delta_{x_{3}, h_{3}}^{\gamma} \mathcal{K}\left(x_{1}, x_{2}, x_{3}\right) \tilde{\phi}\left(R x_{1}\right) \mathrm{d} x_{1}\right| .
\end{aligned}
$$

and

$$
\begin{aligned}
& \left|\int_{\epsilon \leq\left|x_{1}\right| \leq \frac{1}{R}} \Delta_{x_{2}, h_{2}}^{\beta} \Delta_{x_{3}, h_{3}}^{\gamma} \mathcal{K}\left(x_{1}, x_{2}, x_{3}\right) \widetilde{\phi}\left(R x_{1}\right) \mathrm{d} x_{1}\right| \\
& \leq\left|\int_{\epsilon \leq\left|x_{1}\right| \leq \frac{1}{R}} \Delta_{x_{2}, h_{2}}^{\beta} \Delta_{x_{3}, h_{3}}^{\gamma} \mathcal{K}\left(x_{1}, x_{2}, x_{3}\right)\left(\widetilde{\phi}\left(R x_{1}\right)-\widetilde{\phi}(0)\right) \mathrm{d} x_{1}\right| \\
& +\left|\int_{\epsilon \leq\left|x_{1}\right| \leq \frac{1}{R}} \Delta_{x_{2}, h_{2}}^{\beta} \Delta_{x_{3}, h_{3}}^{\gamma} \mathcal{K}\left(x_{1}, x_{2}, x_{3}\right) \tilde{\phi}(0) \mathrm{d} x_{1}\right| \\
& \lesssim \int_{\left|x_{1}\right| \leq \frac{1}{R}} \frac{\left|h_{2}\right|^{\beta \theta_{1}}\left|h_{3}\right|^{\gamma \theta_{1}}}{\left|x_{1}\right|\left|x_{2}\right|^{\beta \theta_{1}+1}\left|x_{3}\right|^{\gamma \theta_{1}+1}}\left(\left|\frac{x_{1} x_{2}}{x_{3}}\right|+\left|\frac{x_{3}}{x_{1} x_{2}}\right|\right)^{-\theta_{2}}\left|R x_{1}\right| \mathrm{d} x_{1} \\
& +\frac{\left|h_{2}\right|^{\beta \theta_{1}}\left|h_{3}\right|^{\gamma \theta_{1}}}{\left|x_{2}\right|^{\beta \theta_{1}+1}\left|x_{3}\right|^{\gamma \theta_{1}+1}}\left(\left|\frac{x_{2}}{R x_{3}}\right|+\left|\frac{R x_{3}}{x_{2}}\right|\right)^{-\theta_{2}} \\
& +\frac{\left|h_{2}\right|^{\beta \theta_{1}}\left|h_{3}\right|^{\gamma \theta_{1}}}{\left|x_{2}\right|^{\beta \theta_{1}+1}\left|x_{3}\right|^{\gamma \theta_{1}+1}}\left(\left|\frac{\epsilon x_{2}}{x_{3}}\right|+\left|\frac{x_{3}}{\epsilon x_{2}}\right|\right)^{-\theta_{2}} \\
& \lesssim \frac{\left|h_{2}\right|^{\beta \theta_{1}}\left|h_{3}\right|^{\gamma \theta_{1}}}{\left|x_{2}\right|^{\beta \theta_{1}+1}\left|x_{3}\right|^{\gamma \theta_{1}+1}}\left(\left|\frac{x_{2}}{R x_{3}}\right|+\left|\frac{R x_{3}}{x_{2}}\right|\right)^{-\theta_{2}} \\
& +\frac{\left|h_{2}\right|^{\beta \theta_{1}}\left|h_{3}\right|^{\gamma \theta_{1}}}{\left|x_{2}\right|^{\beta \theta_{1}+1}\left|x_{3}\right|^{\gamma \theta_{1}+1}}\left(\left|\frac{x_{2}}{\epsilon x_{3}}\right|+\left|\frac{\epsilon x_{3}}{x_{2}}\right|\right)^{-\theta_{2}} .
\end{aligned}
$$

Taking $\epsilon \rightarrow 0$, then (C3.b) is obtained.

Finally we verify (C3.c). For any $0 \leq \alpha \leq 1$, n.b.f. $\tilde{\phi}$ on $\mathbb{R}^{2}$ and $R_{1}, R_{2}>0$, we write

$$
\begin{aligned}
& \left|\iint \Delta_{x_{1}, h_{1}}^{\alpha} \mathcal{K}\left(x_{1}, x_{2}, x_{3}\right) \widetilde{\phi}\left(R_{1} x_{2}, R_{2} x_{3}\right) \mathrm{d} x_{2} \mathrm{~d} x_{3}\right| \\
& \quad=\lim _{\epsilon_{1}, \epsilon_{2} \rightarrow 0}\left|\int_{\epsilon_{2} \leq\left|x_{3}\right| \leq \frac{1}{R_{2}}} \int_{\epsilon_{1} \leq\left|x_{2}\right| \leq \frac{1}{R_{1}}} \Delta_{x_{1}, h_{1}}^{\alpha} \mathcal{K}\left(x_{1}, x_{2}, x_{3}\right) \widetilde{\phi}\left(R_{1} x_{2}, R_{2} x_{3}\right) \mathrm{d} x_{2} \mathrm{~d} x_{3}\right| .
\end{aligned}
$$


and

$$
\begin{aligned}
& \left|\int_{\epsilon_{2} \leq\left|x_{3}\right| \leq \frac{1}{R_{2}}} \int_{\epsilon_{1} \leq\left|x_{2}\right| \leq \frac{1}{R_{1}}} \Delta_{x_{1}, h_{1}}^{\alpha} \mathcal{K}\left(x_{1}, x_{2}, x_{3}\right) \widetilde{\phi}\left(R_{1} x_{2}, R_{2} x_{3}\right) \mathrm{d} x_{2} \mathrm{~d} x_{3}\right| \\
& \leq\left|\int_{\epsilon_{2} \leq\left|x_{3}\right| \leq \frac{1}{R_{2}}} \int_{\epsilon_{1} \leq\left|x_{2}\right| \leq \frac{1}{R_{1}}} \Delta_{x_{1}, h_{1}}^{\alpha} \mathcal{K}\left(x_{1}, x_{2}, x_{3}\right)\left(\widetilde{\phi}\left(R_{1} x_{2}, R_{2} x_{3}\right)-\widetilde{\phi}(0,0)\right) \mathrm{d} x_{2} \mathrm{~d} x_{3}\right| \\
& \quad+\left|\int_{\epsilon_{2} \leq\left|x_{3}\right| \leq \frac{1}{R_{2}}} \int_{\epsilon_{1} \leq\left|x_{2}\right| \leq \frac{1}{R_{1}}} \Delta_{x_{1}, h_{1}}^{\alpha} \mathcal{K}\left(x_{1}, x_{2}, x_{3}\right) \widetilde{\phi}(0,0) \mathrm{d} x_{2} \mathrm{~d} x_{3}\right| \\
& \quad \lesssim \int_{\left|x_{3}\right| \leq \frac{1}{R_{2}}} \int_{\left|x_{2}\right| \leq \frac{1}{R_{1}}} \frac{\left|h_{1}\right|^{\alpha \theta_{1}}}{\left|x_{1}\right|^{\alpha \theta_{1}+1}\left|x_{2}\right|\left|x_{3}\right|}\left(\left|\frac{x_{1} x_{2}}{x_{3}}\right|+\left|\frac{x_{3}}{x_{1} x_{2}}\right|\right)^{-\theta_{2}}\left(\left|R_{1} x_{2}\right|+\left|R_{2} x_{3}\right|\right) \mathrm{d} x_{2} \mathrm{~d} x_{3} \\
& \quad+\frac{\left|h_{1}\right|^{\alpha \theta_{1}}}{\left|x_{1}\right|^{\alpha \theta_{1}+1}} \\
& \quad \frac{\left|h_{1}\right|^{\alpha \theta_{1}}}{\left|x_{1}\right|^{\alpha \theta_{1}+1}} .
\end{aligned}
$$

Thus (C3.c) is obtained. This completes the proof of part (b) and hence Theorem 1.4 is concluded.

\section{Proof of Theorem 1.6}

In this section, we provide the proof of Theorem 1.6. We first study the singular integral $T_{\mathfrak{z}}$ with the convolution kernel as in (1.6), see Theorem 4.1 below. Then we consider the singular integral $T$ with the convolution kernel as in (1.7), see Remark 4.5.

Theorem 4.1 Suppose that $\phi^{(1)}$ and $\phi^{(2)}$ are defined as in (3.1) and

$$
\mathcal{K}\left(x_{1}, x_{2}, x_{3}\right)=\sum_{j, k} 2^{2 j+2 k} \phi^{(1)}\left(2^{j} x_{1}\right) \phi^{(2)}\left(2^{k} x_{2}, 2^{j+k} x_{3}\right) .
$$

Then

$$
\left|\partial_{x_{1}}^{\alpha} \partial_{x_{2}}^{\beta} \partial_{x_{3}}^{\gamma} \mathcal{K}\left(x_{1}, x_{2}, x_{3}\right)\right| \leq \frac{C_{\alpha, \beta, \gamma, \theta_{2}}}{\left|x_{1}\right|^{\alpha+1}\left|x_{2}\right|^{\beta+1}\left|x_{3}\right|^{\gamma+1}}\left(\left|\frac{x_{1} x_{2}}{x_{3}}\right|+\left|\frac{x_{3}}{x_{1} x_{2}}\right|\right)^{-\theta_{2}}
$$

for all $\alpha, \beta, \gamma \geq 0$ and $0<\theta_{2}<1$;

$$
\left|\int_{\delta_{1} \leq\left|x_{1}\right| \leq r_{1}} \int_{\delta_{2} \leq\left|x_{2}\right| \leq r_{2}} \int_{\delta_{3} \leq\left|x_{3}\right| \leq r_{3}} \mathcal{K}\left(x_{1}, x_{2}, x_{3}\right) d x_{1} d x_{2} d x_{3}\right| \leq C
$$


uniformly for all $\delta_{1}, \delta_{2}, \delta_{3}, r_{1}, r_{2}, r_{3}>0$;

$$
\begin{aligned}
& \left|\int_{\delta \leq\left|x_{1}\right| \leq r} \partial_{x_{2}}^{\beta} \partial_{x_{3}}^{\gamma} \mathcal{K}\left(x_{1}, x_{2}, x_{3}\right) d x_{1}\right| \\
& \quad \leq \frac{C_{\beta, \gamma, \theta_{2}}}{\left|x_{1}\right|^{\beta+1}\left|x_{3}\right|^{1+\gamma}}\left(\frac{1}{\left(\left|\frac{r x_{2}}{x_{3}}\right|+\left|\frac{x_{3}}{r x_{2}}\right|\right)^{\theta_{2}}}+\frac{1}{\left(\left|\frac{\delta x_{2}}{x_{3}}\right|+\left|\frac{x_{3}}{\delta x_{2}}\right|\right)^{\theta_{2}}}\right),
\end{aligned}
$$

for all $\delta, r>0, \beta, \gamma \geq 0$ and $0<\theta_{2}<1$;

$$
\left|\int_{\delta_{1} \leq\left|x_{2}\right| \leq r_{1}} \int_{\delta_{2} \leq\left|x_{3}\right| \leq r_{2}} \partial_{x_{1}}^{\alpha} \mathcal{K}\left(x_{1}, x_{2}, x_{3}\right) d x_{2} d x_{3}\right| \leq \frac{C_{\alpha}}{\left|x_{1}\right|^{\alpha+1}}
$$

uniformly for all $\delta_{1}, \delta_{2}, r_{1}, r_{2}>0$ and $\alpha \geq 0$.

To show Theorem 4.1, we need the following simple lemmas.

Lemma 4.2 Suppose $a, b, c>0$ with $b>a$ and $r_{1}, r_{2}, r_{3}>0$. Then, for all $0<\varepsilon<$ 1 ,

$$
\sum_{j} 2^{j a} \frac{1}{\left(1+2^{j} r_{1}\right)^{b}} \frac{1}{\left(r_{2}+2^{j} r_{3}\right)^{c}} \leq C_{\varepsilon} r_{1}^{-a} r_{2}^{-c}\left(1+\frac{r_{3}}{r_{1} r_{2}}\right)^{-(a \wedge c)+1-\varepsilon}
$$

Proof We first write

$$
\begin{aligned}
& \sum_{j} 2^{j a} \frac{1}{\left(1+2^{j} r_{1}\right)^{b}} \frac{1}{\left(r_{2}+2^{j} r_{3}\right)^{c}}=\sum_{j} 2^{j a} \frac{1}{\left(1+2^{j} r_{1}\right)^{b}} \frac{1}{\left(1+2^{j} \frac{r_{3}}{r_{2}}\right)^{c}} \frac{1}{r_{2}^{c}} \\
& \lesssim \sum_{j: 2^{j}>r_{1}^{-1}, 2^{j}>r_{2} r_{3}^{-1}} 2^{j a} \frac{1}{\left(2^{j} r_{1}\right)^{b}} \frac{1}{\left(2^{j} \frac{r_{3}}{r_{2}}\right)^{c}} \frac{1}{r_{2}^{c}}+\sum_{j: 2^{j}>r_{1}^{-1}, 2^{j} \leq r_{2} r_{3}^{-1}} 2^{j a} \frac{1}{\left(2^{j} r_{1}\right)^{b}} \frac{1}{r_{2}^{c}} \\
&+\sum_{j: 2^{j} \leq r_{1}^{-1}, 2^{j}>r_{2} r_{3}^{-1}} 2^{j a} \frac{1}{\left(2^{j} \frac{r_{3}}{r_{2}}\right)^{c}} \frac{1}{r_{2}^{c}}+\sum_{j: 2^{j} \leq r_{1}^{-1}, 2^{j} \leq r_{2} r_{3}^{-1}} 2^{j a} \frac{1}{r_{2}^{c}} \\
&:=I+I I+I I I+I V .
\end{aligned}
$$

For term $I$, we observe that

$$
I \lesssim\left(1+\frac{r_{3}}{r_{1} r_{2}}\right)^{a-b-c} r_{1}^{-a} r_{2}^{-c}\left(\frac{r_{3}}{r_{1} r_{2}}\right)^{b-a} \lesssim r_{1}^{-a} r_{2}^{-c}\left(1+\frac{r_{3}}{r_{1} r_{2}}\right)^{-c}
$$

For $I I$, since $r_{3}<r_{1} r_{2}$,

$$
I I \lesssim r_{1}^{-a} r_{2}^{-c} \lesssim r_{1}^{-a} r_{2}^{-c}\left(1+\frac{r_{3}}{r_{1} r_{2}}\right)^{-c}
$$


For $I I I$, note that $r_{3}>r_{1} r_{2}$. We consider three cases. In the first case where $a>c$, we obtain

$$
I I I \lesssim r_{1}^{-a} r_{2}^{-c}\left(\frac{r_{3}}{r_{1} r_{2}}\right)^{-c} \lesssim r_{1}^{-a} r_{2}^{-c}\left(1+\frac{r_{3}}{r_{1} r_{2}}\right)^{-c}
$$

If $a<c$, then

$$
I I I \lesssim r_{1}^{-a} r_{2}^{-c}\left(\frac{r_{3}}{r_{1} r_{2}}\right)^{-a} \lesssim r_{1}^{-a} r_{2}^{-c}\left(1+\frac{r_{3}}{r_{1} r_{2}}\right)^{-a}
$$

When $a=c$, we have

$$
I I I \lesssim r_{3}^{-c} \log \left(\frac{r_{3}}{r_{1} r_{2}}\right) \lesssim r_{1}^{-a} r_{2}^{-c}\left(1+\frac{r_{3}}{r_{1} r_{2}}\right)^{-a+1-\theta_{2}}
$$

Finally, for term $I V$, we have

$$
I V \lesssim\left(\frac{r_{1} r_{2}}{r_{3}+r_{1} r_{2}}\right)^{a} r_{1}^{-a} r_{2}^{-c}=r_{1}^{-a} r_{2}^{-c}\left(1+\frac{r_{3}}{r_{1} r_{2}}\right)^{-a}
$$

These estimates yield the required bound and Lemma 4.2 is proved.

Lemma 4.3 For any $N>0, r>0$ and $k \in \mathbb{Z}$, we have

$$
\int_{\left\{x_{1} \in \mathbb{R}:\left|x_{1}\right| \leq r\right\}} \frac{1}{\left(1+2^{k}\left|x_{1}\right|\right)^{N}} d x_{1} \lesssim \frac{r}{1+2^{k} r}
$$

and

$$
\int_{\left\{x_{1} \in \mathbb{R}:\left|x_{1}\right|>r\right\}} \frac{1}{\left(1+2^{k}\left|x_{1}\right|\right)^{N}} d x_{1} \lesssim \frac{2^{-k}}{\left(1+2^{k} r\right)^{N-1}}
$$

Proof We consider two cases. For the case $r \leq 2^{-k}$, we clearly have $\int_{\left|x_{1}\right| \leq r} \frac{1}{\left(1+2^{k}\left|x_{1}\right|\right)^{N}} \mathrm{~d} x_{1} \lesssim r$. The second inequality follows from

$$
\int_{\mathbb{R}} \frac{1}{\left(1+2^{k}\left|x_{1}\right|\right)^{N}} \mathrm{~d} x_{1}=2^{-k} \int_{\mathbb{R}} \frac{1}{\left(1+\left|x_{1}\right|\right)^{N}} \mathrm{~d} x_{1} \lesssim 2^{-k} .
$$

If $r>2^{-k}$, then the first inequality follows again from (4.5) while the second follows from

$$
\int_{\mid x_{1}>r} \frac{1}{\left(1+2^{k}\left|x_{1}\right|\right)^{N}} \mathrm{~d} x_{1} \leq \int_{\left|x_{1}\right|>r} \frac{1}{\left(2^{k}\left|x_{1}\right|\right)^{N}} \mathrm{~d} x_{1} \lesssim \frac{2^{-k}}{\left(1+2^{k} r\right)^{N-1}}
$$

The proof of Lemma 4.3 is finished.

We now return to show Theorem 4.1. 
Proof of Theorem 4.1 We prove the regularity estimate (4.1) first. By the definition of $\mathcal{K}$ and the conditions on $\phi^{(1)}$ and $\phi^{(2)}$, we have

$$
\left|\partial_{x_{1}}^{\alpha} \partial_{x_{2}}^{\beta} \partial_{x_{3}}^{\gamma} \mathcal{K}\left(x_{1}, x_{2}, x_{3}\right)\right| \lesssim \sum_{j, k} \frac{2^{2 j+2 k+j(\alpha+\gamma)+k(\beta+\gamma)}}{\left(1+2^{j}\left|x_{1}\right|\right)^{3+\alpha+\gamma}\left(1+2^{k}\left|x_{2}\right|+2^{j+k}\left|x_{3}\right|\right)^{3}}
$$

Note that

$$
\begin{aligned}
\sum_{k} \frac{2^{2 k+k(\beta+\gamma)}}{\left(1+2^{k}\left|x_{2}\right|+2^{j+k}\left|x_{3}\right|\right)^{3}}= & \sum_{k: 2^{k} \leq\left(\left|x_{2}\right|+2^{j}\left|x_{3}\right|\right)^{-1}} \frac{2^{2 k+k(\beta+\gamma)}}{\left(1+2^{k}\left|x_{2}\right|+2^{j+k}\left|x_{3}\right|\right)^{3}} \\
& +\sum_{k: 2^{k}>\left(\left|x_{2}\right|+2^{j}\left|x_{3}\right|\right)^{-1}} \frac{2^{2 k+k(\beta+\gamma)}}{\left(1+2^{k}\left|x_{2}\right|+2^{j+k}\left|x_{3}\right|\right)^{3}} \\
\lesssim & \frac{1}{\left(\left|x_{2}\right|+2^{j}\left|x_{3}\right|\right)^{2+\beta+\gamma}} .
\end{aligned}
$$

Inserting this estimate into the above inequality, we obtain

$$
\begin{aligned}
\left|\partial_{x_{1}}^{\alpha} \partial_{x_{2}}^{\beta} \partial_{x_{3}}^{\gamma} \mathcal{K}\left(x_{1}, x_{2}, x_{3}\right)\right| & \lesssim \sum_{j} \frac{2^{2 j+j(\alpha+\gamma)}}{\left(1+2^{j}\left|x_{1}\right|\right)^{3+\alpha+\gamma}\left(\left|x_{2}\right|+2^{j}\left|x_{3}\right|\right)^{2+\beta+\gamma}} \\
& \lesssim \frac{1}{\left|x_{1}\right|^{\alpha+\gamma+2}\left|x_{2}\right|^{\beta+\gamma+2}\left(1+\left|\frac{x_{3}}{x_{1} x_{2}}\right|\right)^{(\alpha \wedge \beta)+\gamma+1+\theta_{2}}}
\end{aligned}
$$

where we apply Lemma 4.2 with $a=2+\alpha+\gamma, b=3+\alpha+\gamma, c=2+\beta+\gamma, r_{1}=$ $\left|x_{1}\right|, r_{2}=\left|x_{2}\right|$ and $r_{3}=\left|x_{3}\right|$ in the last inequality. This implies the required estimate.

We now show the cancellation conditions (4.2)-(4.4). To verify (4.3), we observe that

$$
\begin{aligned}
& \left|\int_{\delta \leq\left|x_{1}\right| \leq r} \partial_{x_{2}}^{\beta} \partial_{x_{3}}^{\gamma} \mathcal{K}\left(x_{1}, x_{2}, x_{3}\right) \mathrm{d} x_{1}\right| \\
& \quad \lesssim \sum_{j, k} 2^{2 j+2 k+k \beta+(j+k) \gamma}\left|\int_{\delta \leq\left|x_{1}\right| \leq r} \phi^{(1)}\left(2^{j} x_{1}\right)\left(\partial_{x_{2}}^{\beta} \partial_{x_{3}}^{\gamma} \phi^{(2)}\right)\left(2^{k} x_{2}, 2^{j+k} x_{3}\right) \mathrm{d} x_{1}\right| .
\end{aligned}
$$

Note that for all $N \geq 2$,

$$
\left|\int_{\left|x_{1}\right| \leq r} \phi^{(1)}\left(2^{j} x_{1}\right) \mathrm{d} x_{1}\right| \lesssim r
$$


and by the vanishing condition of $\phi^{(1)}$,

$$
\begin{aligned}
\left|\int_{\left|x_{1}\right| \leq r} \phi^{(1)}\left(2^{j} x_{1}\right) \mathrm{d} x_{1}\right| & =\left|\int_{\left|x_{1}\right|>r} \phi^{(1)}\left(2^{j} x_{1}\right) \mathrm{d} x_{1}\right| \\
& \leq C_{N}\left|\int_{\left|x_{1}\right|>r} \frac{1}{\left(2^{j}\left|x_{1}\right|\right)^{N}} \mathrm{~d} x_{1}\right| \leq C_{N} 2^{-j N} r^{1-N} .
\end{aligned}
$$

Therefore,

$$
\left|\int_{\left|x_{1}\right| \leq r} \phi^{(1)}\left(2^{j} x_{1}\right) \mathrm{d} x_{1}\right| \leq C_{N} \frac{r}{\left(1+2^{j} r\right)^{N}}
$$

which implies

$$
\begin{aligned}
\left|\int_{\delta \leq\left|x_{1}\right| \leq r} \partial_{x_{2}}^{\beta} \partial_{x_{3}}^{\gamma} \mathcal{K}\left(x_{1}, x_{2}, x_{3}\right) \mathrm{d} x_{1}\right| \lesssim & \sum_{j, k} \frac{2^{2 j+2 k+j \gamma+k(\beta+\gamma)} r}{\left(1+2^{j} r\right)^{3+\gamma}\left(1+2^{k}\left|x_{2}\right|+2^{j+k}\left|x_{3}\right|\right)^{3}} \\
& +\sum_{j, k} \frac{2^{2 j+2 k+j \gamma+k(\beta+\gamma)} \delta}{\left(1+2^{j} \delta\right)^{3+\gamma}\left(1+2^{k}\left|x_{2}\right|+2^{j+k}\left|x_{3}\right|\right)^{3}} .
\end{aligned}
$$

Summing over $k$ first yields that the two summations above are dominated by

$$
\sum_{j}\left(\frac{r}{\left(1+2^{j} r\right)^{3+\gamma}}+\frac{\delta}{\left(1+2^{j} \delta\right)^{3+\gamma}}\right) \frac{2^{2 j+j \gamma}}{\left(\left|x_{2}\right|+2^{j}\left|x_{3}\right|\right)^{2+\beta+\gamma}} .
$$

Applying Lemma 4.2 with $a=2+\gamma, b=3+\gamma, c=2+\gamma+\beta, r_{1}=r$ or $\delta$, $r_{2}=\left|x_{2}\right|, r_{3}=\left|x_{3}\right|$, we obtain

$$
\begin{aligned}
& \left|\int_{\delta \leq\left|x_{1}\right| \leq r} \partial_{x_{2}}^{\beta} \partial_{x_{3}}^{\gamma} \mathcal{K}\left(x_{1}, x_{2}, x_{3}\right) \mathrm{d} x_{1}\right| \\
& \quad \lesssim \frac{1}{r^{\gamma+1}\left|x_{2}\right|^{\beta+\gamma+2}\left(1+\left|\frac{x_{3}}{r x_{2}}\right|\right)^{\gamma+1+\theta_{2}}}+\frac{1}{\delta \gamma+1\left|x_{2}\right|^{\beta+\gamma+2}\left(1+\left|\frac{x_{3}}{\delta x_{2}}\right|\right)^{\gamma+1+\theta_{2}}}
\end{aligned}
$$

which implies the desired cancellation condition (4.3).

To show the cancellation condition (4.4), we start with

$$
\begin{aligned}
& \left|\int_{\delta_{1} \leq\left|x_{2}\right| \leq r_{1}} \int_{\delta_{2} \leq\left|x_{3}\right| \leq r_{2}} \partial_{x_{1}}^{\alpha} \mathcal{K}\left(x_{1}, x_{2}, x_{3}\right) \mathrm{d} x_{2} \mathrm{~d} x_{3}\right| \\
& \lesssim \sum_{j, k} 2^{2 j+2 k+j \alpha}\left|\int_{\left|x_{2}\right| \leq r_{1}} \int_{\left|x_{3}\right| \leq r_{2}}\left(\partial_{x_{1}}^{\alpha} \phi^{(1)}\right)\left(2^{j} x_{1}\right) \phi^{(2)}\left(2^{k} x_{2}, 2^{j+k} x_{3}\right) \mathrm{d} x_{2} \mathrm{~d} x_{3}\right| \\
& \quad+\sum_{j, k} 2^{2 j+2 k+j \alpha}\left|\int_{\left|x_{2}\right| \leq \delta_{1}} \int_{\left|x_{3}\right| \leq \delta_{2}}\left(\partial_{x_{1}}^{\alpha} \phi^{(1)}\right)\left(2^{j} x_{1}\right) \phi^{(2)}\left(2^{k} x_{2}, 2^{j+k} x_{3}\right) \mathrm{d} x_{2} \mathrm{~d} x_{3}\right| .
\end{aligned}
$$


By the vanishing condition of $\phi^{(2)}$,

$$
\begin{aligned}
& \int_{\left|x_{2}\right| \leq r_{1}} \int_{\left|x_{3}\right| \leq r_{2}} \phi^{(2)}\left(2^{k} x_{2}, 2^{j+k} x_{3}\right) \mathrm{d} x_{2} \mathrm{~d} x_{3} \\
& =\int_{\left|x_{2}\right|>r_{1}} \int_{\left|x_{3}\right| \leq r_{2}} \phi^{(2)}\left(2^{k} x_{2}, 2^{j+k} x_{3}\right) \mathrm{d} x_{2} \mathrm{~d} x_{3} \\
& \quad+\int_{\left|x_{2}\right| \leq r_{1}} \int_{\left|x_{3}\right|>r_{2}} \phi^{(2)}\left(2^{k} x_{2}, 2^{j+k} x_{3}\right) \mathrm{d} x_{2} \mathrm{~d} x_{3} \\
& \quad+\int_{\left|x_{2}\right|>r_{1}} \int_{\left|x_{3}\right|>r_{2}} \phi^{(2)}\left(2^{k} x_{2}, 2^{j+k} x_{3}\right) \mathrm{d} x_{2} \mathrm{~d} x_{3} .
\end{aligned}
$$

Applying the size condition of $\phi^{(2)}$ and Lemma 4.3, we obtain

$$
\begin{aligned}
& \left|\int_{\left|x_{2}\right| \leq r_{1}} \int_{\left|x_{3}\right| \leq r_{2}} \phi^{(2)}\left(2^{k} x_{2}, 2^{j+k} x_{3}\right) \mathrm{d} x_{2} \mathrm{~d} x_{3}\right| \\
& \quad \lesssim \frac{2^{-k}}{\left(1+2^{k} r_{1}\right)^{3}} \frac{r_{2}}{1+2^{j+k} r_{2}}+\frac{r_{1}}{1+2^{k} r_{1}} \frac{2^{-j-k}}{\left(1+2^{j+k} r_{2}\right)^{3}}+\frac{2^{-k}}{\left(1+2^{k} r_{1}\right)^{3}} \frac{2^{-j-k}}{\left(1+2^{j+k} r_{2}\right)^{3}} .
\end{aligned}
$$

On other hand, by the size condition on $\phi^{(2)}$,

$$
\left|\int_{\left|x_{2}\right| \leq r_{1}} \int_{\left|x_{3}\right| \leq r_{2}} \phi^{(2)}\left(2^{k} x_{2}, 2^{j+k} x_{3}\right) \mathrm{d} x_{2} d x_{3}\right| \leq \frac{r_{1}}{1+2^{k} r_{1}} \frac{r_{2}}{1+2^{j+k} r_{2}} .
$$

Therefore,

$$
\begin{aligned}
& \sum_{j, k} 2^{2 j+2 k+j \alpha \mid}\left|\left(\partial_{x_{1}} \phi^{(1)}\right)\left(2^{j} x_{1}\right) \int_{\left|x_{2}\right| \leq r_{1}} \int_{\left|x_{3}\right| \leq r_{2}} \phi^{(2)}\left(2^{k} x_{2}, 2^{j+k} x_{3}\right)\left(x_{2}, x_{3}\right) \mathrm{d} x_{2} \mathrm{~d} x_{3}\right| \\
& \lesssim \sum_{j, k} \frac{2^{2 j+2 k+j \alpha}}{\left(1+2^{j}\left|x_{1}\right|\right)^{3+\alpha}} \min \left\{\left(\frac{2^{-k}}{\left(1+2^{k} r_{1}\right)^{3}} \frac{r_{2}}{1+2^{j+k} r_{2}}+\frac{r_{1}}{1+2^{k} r_{1}} \frac{2^{-j-k}}{\left(1+2^{j+k} r_{2}\right)^{3}}\right.\right. \\
& \left.\left.\quad+\frac{2^{-k}}{\left(1+2^{k} r_{1}\right)^{3}} \frac{2^{-j-k}}{\left(1+2^{j+k} r_{2}\right)^{3}}\right), \frac{r_{1}}{1+2^{k} r_{1}} \frac{r_{2}}{1+2^{j+k} r_{2}}\right\} .
\end{aligned}
$$

Summing over $k$ first and considering the four cases: (i) $2^{k} \leq r_{1}^{-1}$ and $2^{k} \leq 2^{-j} r_{2}^{-1}$; (ii) $2^{k} \leq r_{1}^{-1}$ and $2^{k}>2^{-j} r_{2}^{-1}$; (iii) $2^{k}>r_{1}^{-1}$ and $2^{k} \leq 2^{-j} r_{2}^{-1}$; (iv) $2^{k}>r_{1}^{-1}$ and $2^{k}>2^{-j} r_{2}^{-1}$, we obtain that the last summation above is dominated by

$$
\sum_{j} \frac{2^{2 j+j \alpha}}{\left(1+2^{j}\left|x_{1}\right|\right)^{3+\alpha}} 2^{-j}
$$

which yields the cancellation condition (4.4). 
Finally, the cancellation (4.2) follows directly from the following estimates.

$$
\begin{aligned}
& \left|\int_{\delta_{1} \leq\left|x_{1}\right| \leq r_{1}} \int_{\delta_{2} \leq\left|x_{2}\right| \leq r_{2}} \int_{\delta_{3} \leq\left|x_{3}\right| \leq r_{3}} \mathcal{K}\left(x_{1}, x_{2}, x_{3}\right) \mathrm{d} x_{1} \mathrm{~d} x_{2} \mathrm{~d} x_{3}\right| \\
& \quad \lesssim \mid \sum_{j, k} 2^{2 j+2 k} \int_{\delta_{1} \leq\left|x_{1}\right| \leq r_{1}} \phi^{(1)}\left(2^{j} x_{1}\right) \mathrm{d} x_{1} \\
& \quad \times \int_{\delta_{2} \leq\left|x_{2}\right| \leq r_{2}} \int_{\delta_{3} \leq\left|x_{3}\right| \leq r_{3}} \phi^{(2)}\left(2^{k} x_{2}, 2^{j+k} x_{3}\right)\left(x_{2}, x_{3}\right) \mathrm{d} x_{2} \mathrm{~d} x_{3} \mid \\
& \quad \lesssim \sum_{j} 2^{2 j}\left(\frac{r_{1}}{\left(1+2^{j} r_{1}\right)^{3}} 2^{-j}+\frac{\delta_{1}}{\left(1+2^{j} \delta_{1}\right)^{3}} 2^{-j}\right) \lesssim 1 .
\end{aligned}
$$

The proof of Theorem 4.1 is complete.

As mentioned in Sect. 1, a special class of singular integral operators $T_{\mathfrak{z}}$ considered by Ricci and Stein [28] is of the form $T_{\mathfrak{z}} f=f * \mathcal{K}$, where

$$
\mathcal{K}\left(x_{1}, x_{2}, x_{3}\right)=\sum_{k, j \in \mathbb{Z}} 2^{2(k+j)} \phi\left(2^{j} x_{1}, 2^{k} x_{2}, 2^{j+k} x_{3}\right)
$$

and the function $\phi$ is supported in an unit cube in $\mathbb{R}^{3}$ and satisfies a certain amount of uniform smoothness with cancellation conditions

$$
\int_{\mathbb{R}^{2}} \phi\left(x_{1}, x_{2}, x_{3}\right) \mathrm{d} x_{1} \mathrm{~d} x_{2}=\int_{\mathbb{R}^{2}} \phi\left(x_{1}, x_{2}, x_{3}\right) \mathrm{d} x_{2} \mathrm{~d} x_{3}=\int_{\mathbb{R}^{2}} \phi\left(x_{1}, x_{2}, x_{3}\right) \mathrm{d} x_{3} \mathrm{~d} x_{1}=0 .
$$

Fefferman and Pipher [13] show that the above cancellation conditions are necessary for the $L^{2}$ boundedness for singular integral $T_{\mathfrak{z}}$. Moreover, if $\phi$ satisfies the above cancellation conditions, then $\phi$ can be decomposed by $\phi=\phi_{1}+\phi_{2}$, where $\phi_{1}$ and $\phi_{2}$ have the following cancellation conditions

$$
\int_{\mathbb{R}} \phi_{1}\left(x_{1}, x_{2}, x_{3}\right) \mathrm{d} x_{1}=\int_{\mathbb{R}^{2}} \phi_{1}\left(x_{1}, x_{2}, x_{3}\right) \mathrm{d} x_{2} \mathrm{~d} x_{3}=0
$$

and

$$
\int_{\mathbb{R}} \phi_{2}\left(x_{1}, x_{2}, x_{3}\right) \mathrm{d} x_{2}=\int_{\mathbb{R}^{2}} \phi_{2}\left(x_{1}, x_{2}, x_{3}\right) \mathrm{d} x_{1} \mathrm{~d} x_{3}=0 .
$$

This means that the operator $T_{\mathfrak{z}}$ studied by Ricci and Stein can be decomposed as $T_{\mathfrak{z}}=T_{\mathfrak{z}, 1}+T_{\mathfrak{z}, 2}$, where the kernels of $T_{\mathfrak{z}, 1}$ and $T_{\mathfrak{z}, 2}$ are given, respectively, by

$$
\mathcal{K}_{1}\left(x_{1}, x_{2}, x_{3}\right)=\sum_{j, k \in \mathbb{Z}} 2^{2(k+j)} \phi_{1}\left(2^{j} x_{1}, 2^{k} x_{2}, 2^{j+k} x_{3}\right)
$$


and

$$
\mathcal{K}_{2}\left(x_{1}, x_{2}, x_{3}\right)=\sum_{j, k \in \mathbb{Z}} 2^{2(k+j)} \phi_{2}\left(2^{j} x_{1}, 2^{k} x_{2}, 2^{j+k} x_{3}\right) .
$$

Theorem 4.1 shows that the kernel $\mathcal{K}_{1}$ satisfies the regularity (RR) and cancellation conditions (C2.a)-(C2.c) while the kernel $\mathcal{K}_{2}$ satisfies the regularity (RR) and cancellation conditions $\left(\mathrm{C} 2^{\prime}\right.$.a $)-\left(\mathrm{C} 2^{\prime}\right.$.c $)$. Therefore, these operators $\mathcal{K}_{1}$ and $\mathcal{K}_{2}$ belong to our class.

Remark 4.4 Actually, based on the proof of Theorem 4.1, we note that the kernel

$$
\mathcal{K}\left(x_{1}, x_{2}, x_{3}\right)=\sum_{j, k \in \mathbb{Z}} 2^{2 j+2 k} \phi^{(1)}\left(2^{j} x_{1}\right) \phi^{(2)}\left(2^{k} x_{2}, 2^{j+k} x_{3}\right)
$$

as in Theorem 4.1 satisfies the following stronger conditions:

$$
\begin{aligned}
& \left|\partial_{x_{1}}^{\alpha} \partial_{x_{2}}^{\beta} \partial_{x_{3}}^{\gamma} \mathcal{K}\left(x_{1}, x_{2}, x_{3}\right)\right| \leq \frac{C_{\alpha, \beta, \gamma, \theta_{2}}}{\left|x_{1}\right|^{\alpha+\gamma+2}\left|x_{2}\right|^{\beta+\gamma+2}\left(1+\left|\frac{x_{3}}{x_{1} x_{2}}\right|\right)^{(\alpha \wedge \beta)+\gamma+1+\theta_{2}}} ; \\
& \left|\int_{\delta_{1} \leq\left|x_{1}\right| \leq r_{1}} \int_{\delta_{2} \leq\left|x_{2}\right| \leq r_{2}} \int_{\delta_{3} \leq\left|x_{3}\right| \leq r_{3}} \mathcal{K}\left(x_{1}, x_{2}, x_{3}\right) \mathrm{d} x_{1} \mathrm{~d} x_{2} \mathrm{~d} x_{3}\right| \leq C
\end{aligned}
$$

uniformly for all $\delta_{1}, \delta_{2}, \delta_{3}, r_{1}, r_{2}, r_{3}>0$;

$$
\begin{aligned}
& \left|\int_{\delta \leq\left|x_{1}\right| \leq r} \partial_{x_{2}}^{\beta} \partial_{x_{3}}^{\gamma} \mathcal{K}\left(x_{1}, x_{2}, x_{3}\right) \mathrm{d} x_{1}\right| \\
& \quad \leq C_{\beta, \gamma, \theta_{2}}\left(\frac{1}{r^{\gamma+1}\left|x_{2}\right|^{\beta+\gamma+2}\left(1+\left|\frac{x_{3}}{r x_{2}}\right|\right)^{\gamma+1+\theta_{2}}}+\frac{1}{\delta^{\gamma+1}\left|x_{2}\right|^{\beta+\gamma+2}\left(1+\left|\frac{x_{3}}{\delta x_{2}}\right|\right)^{\gamma+1+\theta_{2}}}\right)
\end{aligned}
$$

for all $\delta, r>0, \beta, \gamma \geq 0$ and $0<\theta_{2}<1$ and

$$
\left|\int_{\delta_{1} \leq\left|x_{2}\right| \leq r_{1}} \int_{\delta_{2} \leq\left|x_{3}\right| \leq r_{2}} \partial_{x_{1}}^{\alpha} \mathcal{K}\left(x_{1}, x_{2}, x_{3}\right) \mathrm{d} x_{2} \mathrm{~d} x_{3}\right| \leq \frac{C_{\alpha}}{\left|x_{1}\right|^{\alpha+1}}
$$

uniformly for all $\delta_{1}, \delta_{2}, r_{1}, r_{2}>0$ and $\alpha \geq 0$.

Remark 4.5 In [23], Nagel and Wainger considered the $L^{2}$ boundedness of certain singular integral operators on $\mathbb{R}^{n}$ whose kernels have appropriate homogeneities with respect to a multi-parameter group of dilations, generated by a finite number of diagonal matrices. In particular, they considered the following two-parameter dilation group

$$
\delta(s, t)\left(x_{1}, x_{2}, x_{3}\right)=\left(s x_{1}, t x_{2}, s^{\alpha} t^{\beta} x_{3}\right)
$$


acting on $\mathbb{R}^{3}$ for $s, t, \alpha, \beta>0$. They defined a singular kernel $\mathcal{K}$ by

$$
\mathcal{K}\left(x_{1}, x_{2}, x_{3}\right)=\operatorname{sgn}\left(x_{1} x_{2}\right)\left\{\frac{\left|x_{1}\right|^{\alpha-1}\left|x_{2}\right|^{\beta-1}}{\left|x_{1}\right|^{2 \alpha}\left|x_{2}\right|^{2 \beta}+x_{3}^{2}}\right\}
$$

and proved that convolution with $\mathcal{K}$ is bounded on $L^{2}\left(\mathbb{R}^{3}\right)$.

It is easy to see that when $\alpha=\beta=1, \mathcal{K}\left(x_{1}, x_{2}, x_{3}\right)$ satisfies all conditions in Corollary 1.2 and Theorem 1.3. Therefore, by Theorem 1.3, the convolution singular integral operator $\mathcal{K} * f$ with $\alpha=\beta=1$ is also bounded on $L^{p}\left(\mathbb{R}^{3}\right)$ for $1<p<\infty$, where $\mathcal{K} * f$ is defined by the limit of $\mathcal{K}_{\epsilon}^{N} * f$ in the $L^{p}, 1<p<\infty$, norm. It is worthwhile to point out that the theory we are developing here can easily be generalized to the 'anisotropic' case (adapted to $\delta(s, t)$ in (4.6)). The details are left to the interested reader.

Open Access This article is distributed under the terms of the Creative Commons Attribution 4.0 International License (http://creativecommons.org/licenses/by/4.0/), which permits unrestricted use, distribution, and reproduction in any medium, provided you give appropriate credit to the original author(s) and the source, provide a link to the Creative Commons license, and indicate if changes were made.

\section{References}

1. Calderón, A.P., Zygmund, A.: On the existence of certain singular integrals. Acta Math. 88, 85-139 (1952)

2. Carleson, L.: A counterexample for measures bounded on $H^{p}$ for the bidisc. Mittag-Leffler Report No. 7 (1974)

3. Chang, S.-Y.A.: Carleson measures on the bi-disc. Ann. Math. 109, 613-620 (1979)

4. Chang, S.-Y.A., Fefferman, R.: A continuous version of duality of $H^{1}$ with $B M O$ on the bidisc. Ann. Math. 112, 179-201 (1980)

5. Chang, S.-Y.A., Fefferman, R.: The Calderón-Zygmund decomposition on product domains. Am. J. Math. 104, 455-468 (1982)

6. Chang, S.-Y.A., Fefferman, R.: Some recent developments in Fourier analysis and $H^{p}$ theory on product domains. Bull. Am. Math. Soc. 12, 1-43 (1985)

7. Cordoba, A., Fefferman, R.: A geometric proof of the strong maximal theorem. Ann. Math. 102, 95-100 (1975)

8. Fefferman, C., Stein, E.M.: Some maximal inequalities. Am. J. Math. 93, 107-115 (1971)

9. Fefferman, R.: Some weighted norm inequalities for Cordoba's maximal functions. Am. J. Math. 106, 1261-1264 (1984)

10. Fefferman, R.: Multi-parameter Fourier analysis. In: Stein, E.M. (ed.) Beijing Lectures in Harmonic Analysis. Annals of Mathematics Studies, vol. 112, pp. 47-130. Princeton University Press, Princeton (1986)

11. Fefferman, R.: Harmonic analysis on product spaces. Ann. Math. 126, 109-130 (1987)

12. Fefferman, R.: Multiparameter Calderón-Zygmund theory. In: Harmonic Analysis and Partial Differential Equations (Chicago, IL, 1996). Chicago Lectures in Mathematics, pp. 207-221. University of Chicago Press, Chicago (1999)

13. Fefferman, R., Pipher, J.: Multiparameter operators and sharp weighted inequalities. Am. J. Math. 11, 337-369 (1997)

14. Fefferman, R., Stein, E.M.: Singular integrals on product spaces. Adv. Math. 45, 117-143 (1982)

15. Ferguson, S.H., Lacey, M.T.: A characterization of product BMO by commutators. Acta Math. 189, 143-160 (2002)

16. Gundy, R., Stein, E.M.: $H^{p}$ theory for the polydisk. Proc. Natl Acad Sci. U.S.A. 76, 1026-1029 (1979) 
17. Jessen, B., Marcinkiewicz, J., Zygmund, A.: Note on the differentiability of multiple integrals. Fundam. Math. 25, 217-234 (1935)

18. Journé, J.L.: Calderón-Zygmund operators on product spaces. Rev. Mat. Iberoam. 1, 55-92 (1985)

19. Journé, J.L.: A covering lemma for product spaces. Proc. Am. Math. Soc. 96, 593-598 (1986)

20. Journé, J.L.: Two problems of Calderón-Zygmund theory on product spaces. Ann. Inst. Fourier (Grenoble) 38, 111-132 (1988)

21. Lacey, M., Petermichl, S., Pipher, J., Wick, B.D.: Multiparameter Riesz commutators. Am. J. Math. 131, 731-769 (2009)

22. Müller, D., Ricci, F., Stein, E.M.: Marcinkiewicz multipliers and multi-parameter structure on Heisenberg(-type) groups, I. Invent. Math. 119, 119-233 (1995)

23. Nagel, A., Wainger, S.: $L^{2}$ boundedness of Hilbert transforms along surfaces and convolution operators homogeneous with respect to a multiple parameter group. Am. J. Math. 99, 761-785 (1977)

24. Nagel, A., Ricci, F., Stein, E.M.: Singular integrals with flag kernels and analysis on quadratic CR manifolds. J. Funct. Anal. 181, 29-118 (2001)

25. Nagel, A., Ricci, F., Stein, E.M., Wainger, S.: Singular integrals with flag kernels on homogeneous groups, I. Rev. Mat. Iberoam. 28, 631-722 (2012)

26. Nagel, A., Ricci, F., Stein, E.M., Wainger, S.: Algebras of singular integral operators with kernels controlled by multiple norms. arXiv: 1511.05702

27. Pipher, J.: Journé's covering lemma and its extension to higher dimensions. Duke Math. J. 53, 683-690 (1986)

28. Ricci, F., Stein, E.M.: Multiparameter singular integrals and maximal functions. Ann. Inst. Fourier (Grenoble) 42, 637-670 (1992)

29. Stein, E.M.: Harmonic Analysis: Real-Variable Methods, Orthogonality, and Oscillatory Integrals. Princeton University Press, Princeton (1993) 